Supporting Information for

\title{
Highly Enantioselective Friedel-Crafts Reaction of Indoles with Imines by a Chiral
}

Phosphoric Acid

\section{Qiang Kang, Zhuo-An Zhao and Shu-Li You*}

State Key Laboratory of Organometallic Chemistry

Shanghai Institute of Organic Chemistry, Chinese Academy of Sciences

354 Fenglin Lu, Shanghai 200032, China

Fax: (+86) 21-54925087

E-mail: slyou@mail.sioc.ac.cn

General Methods. Unless stated otherwise, all reactions were carried out in flame-dried glassware under a dry argon atmosphere. All solvents were purified and dried according to standard methods prior to use.

${ }^{1} \mathrm{H}$ and ${ }^{13} \mathrm{C}$ NMR spectra were recorded on a Varian instrument (300 MHz and $75 \mathrm{MHz}$, respectively) and internally referenced to tetramethylsilane signal or residual protio solvent signals. Data for ${ }^{1} \mathrm{H}$ NMR are recorded as follows: chemical shift $(\delta, \mathrm{ppm})$, multiplicity $(\mathrm{s}=$ singlet, $\mathrm{d}=$ doublet, $\mathrm{t}=$ triplet, $\mathrm{m}=$ multiplet or unresolved, br = broad singlet, coupling constant(s) in Hz, integration). Data for ${ }^{13} \mathrm{C} N M R$ are reported in terms of chemical shift $(\delta, \mathrm{ppm})$.

\section{Synthesis of Catalyst:}<smiles>[R]C1=C2OP(=O)(O)OC(=C2c2cc([R])cc3ccccc23)C=c2ccccc2=C1</smiles>

(S)
1a: $\mathrm{R}=\mathrm{H}$
1b: $R=$ Phenyl
1c: $\mathrm{R}=\mathrm{SiPh}_{3}$
1d: $\mathrm{R}=3,5-\mathrm{CF}_{3}-\mathrm{Ph}$
1e: $\mathrm{R}=4-\mathrm{NO}_{2}-\mathrm{Ph}$
1f: $R=1-$ Naphthyl

Catalyst 1a was purchased from Aldrich and used as supplied.

Catalyst $\mathbf{1} \mathbf{b}^{1}, \mathbf{1 c}^{2}, \mathbf{1} \mathbf{d}^{3}, \mathbf{1} \mathbf{e}^{1}, \mathbf{1} \mathbf{f}^{1}$ were prepared according to the reported procedures.

(1) Uraguchi, D.; Terada, M. J. Am. Chem. Soc. 2004, 126, 5356-5357.

(2) Storer, R. I.; Carrera, D. E.; Ni, Y.; MacMillan, D.W. C. J. Am. Chem. Soc. 2006, 128, 84-86.

(3) Akiyama, T.; Morita, H.; Itoh, J.; Fuchibe, K. Org. Lett. 2005, 7, 2583-2585. 


\section{General Procedure for the Catalytic Asymmetric Friedel-Crafts Reaction:}

In a dry Schlenk tube, $N$-sulfonyl imines $3(0.25 \mathrm{mmol})$ and phosphoric acid $\mathbf{1}(0.025 \mathrm{mmol})$ were dissolved in toluene $(1 \mathrm{~mL})$ under argon. The solution was stirred for 10 minutes at room temperature and then for another 5 minutes at $-60 \square$. Subsequently, indoles $2(1.25 \mathrm{mmol})$ were added in one portion at $-60 \square$. After the reaction was complete (monitored by TLC), $10 \% \mathrm{NaHCO}_{3}(3 \mathrm{~mL})$ was added to quench the reaction. The mixture was extracted with ethyl acetate $(10 \mathrm{~mL})$. The organic layer was washed by $\mathrm{H}_{2} \mathrm{O}(5$ $\mathrm{mL}$ ) and brine $(5 \mathrm{~mL})$, separated, and dried over anhydrous $\mathrm{Na}_{2} \mathrm{SO}_{4}$. The solvents were removed under reduced pressure and the residue was purified by flash chromatography (ethyl acetate/petroleum ether $=$ 1/3) to afford the product.

Table 1. Screening the ratios between indole and imine in asmmetric Friedel-Crafts reaction with phosphoric acid 1d.

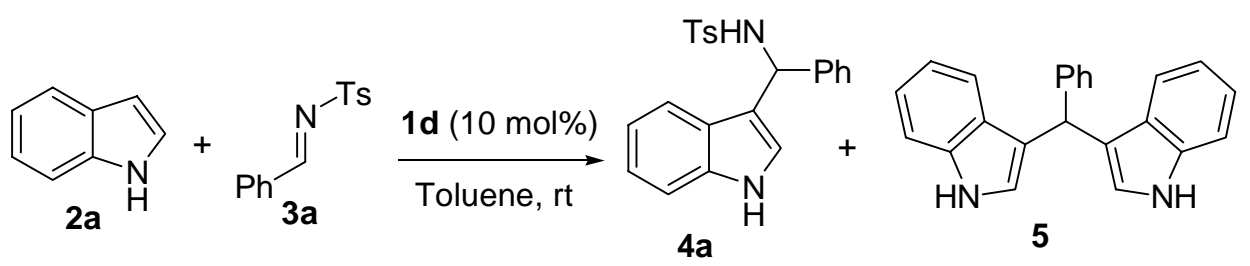

\begin{tabular}{ccccc}
\hline Entry $^{\mathrm{a}}$ & $\mathbf{2 a} / \mathbf{3 a}$ & Time & ${\text { Yield of } \mathbf{4 a}(\%)^{\mathrm{b}}}^{\text {ee of } \mathbf{4 a}(\%)^{\mathrm{c}}}$ \\
\hline 1 & $1 / 1$ & $1 \mathrm{~h}$ & 59 & 67 \\
2 & $2 / 1$ & $30 \mathrm{~min}$ & 75 & 80 \\
3 & $3 / 1$ & $30 \mathrm{~min}$ & 84 & 80 \\
4 & $4 / 1$ & $30 \mathrm{~min}$ & 77 & 83 \\
5 & $5 / 1$ & $30 \mathrm{~min}$ & 80 & 83 \\
\hline
\end{tabular}

\footnotetext{
${ }^{\mathrm{a}}$ Reaction conditions: $10 \mathrm{~mol} \% \mathrm{1d}, 0.25 \mathrm{~mol} / \mathrm{L}$ of 3a in toluene at room temperature. ${ }^{\mathrm{b}}$ Isolated yields. ${ }^{\mathrm{c}}$ Determined by chiral HPLC analysis (Chiralcel OD-H).
} 


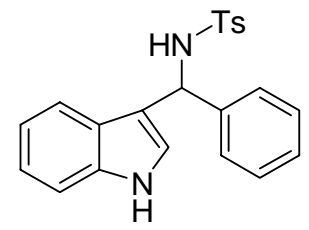

(entry 1, Table 3): $\mathrm{R}_{\mathrm{f}}=0.40$ (ethyl acetate/petroleum ether $=1 / 2$, v/v); colorless solid, $83 \%$ yield, $98 \%$ ee [Daicel Chiralcel OD-H, Hexanes / IPA $=70 / 30,0.6 \mathrm{ml} \square \mathrm{min}^{-1}, \lambda=254 \mathrm{~nm}, \mathrm{t}$ (major) $=16.92 \mathrm{~min}, \mathrm{t}$ $($ minor $)=32.54 \mathrm{~min}] ;[\alpha]_{\mathrm{D}}{ }^{20}=+15.3^{\circ}(\mathrm{c}=0.52$, Acetone $) .{ }^{1} \mathrm{H}$ NMR $\left(300 \mathrm{MHz}, \mathrm{CDCl}_{3}\right) \delta 2.34(\mathrm{~s}, 3 \mathrm{H}), 5.24$ $(\mathrm{d}, J=7.2 \mathrm{~Hz}, 1 \mathrm{H}), 5.82(\mathrm{~d}, J=6.9 \mathrm{~Hz}, 1 \mathrm{H}), 6.61(\mathrm{~d}, J=2.4 \mathrm{~Hz}, 1 \mathrm{H}), 6.97(\mathrm{t}, J=7.8 \mathrm{~Hz}, 1 \mathrm{H}), 7.06(\mathrm{~d}, J=$ $7.8 \mathrm{~Hz}, 2 \mathrm{H}), 7.11-7.27(\mathrm{~m}, 8 \mathrm{H}), 7.53(\mathrm{~d}, J=8.4 \mathrm{~Hz}, 2 \mathrm{H}), 8.02(\mathrm{br}, 1 \mathrm{H}) ;{ }^{13} \mathrm{C}$ NMR $\left(75 \mathrm{MHz}, \mathrm{DMSO}-\mathrm{d}_{6}\right) \delta$ 20.8, 54.4, 111.4, 115.7, 118.8, 118.9, 121.2, 123.7, 125.4, 126.4, 126.6, 127.0, 127.8, 128.9, 136.3, 138.8, $141.7,141.8$. 


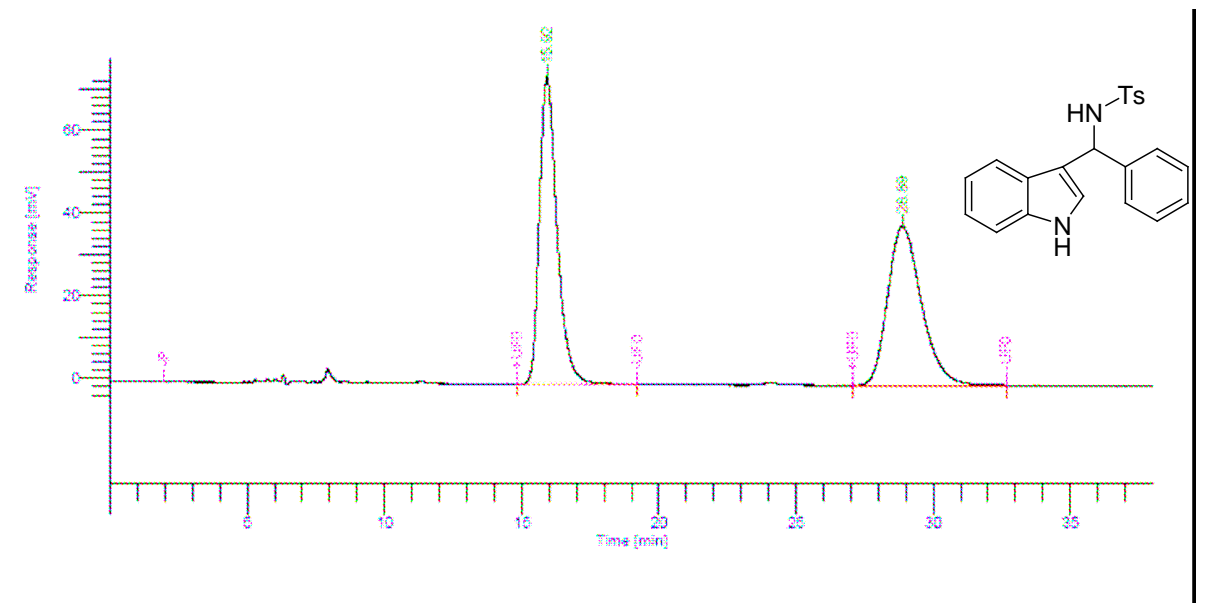

\begin{tabular}{|c|c|c|c|c|c|}
\hline $\begin{array}{c}\text { Peak } \\
\#\end{array}$ & $\begin{array}{l}\text { Time } \\
\text { [min] }\end{array}$ & $\begin{array}{c}\text { Area } \\
{\left[\mathrm{u}^{*} \mathrm{sec}\right]}\end{array}$ & $\begin{array}{c}\text { Height } \\
\text { [uV] }\end{array}$ & $\begin{array}{c}\text { Area } \\
{[\%]}\end{array}$ & $\begin{array}{c}\text { Norm, Area } \\
{[\%]}\end{array}$ \\
\hline \multirow{3}{*}{ 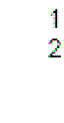 } & 15.92 & $3.3033 e+06$ & $7.435 \mathrm{e}+04$ & 50.05 & 50.05 \\
\hline & 28.89 & $32973 e+06$ & $3.856 \mathrm{e}+04$ & 49.95 & 49.95 \\
\hline & & 6.6006 & 1.129 & 100.00 & 100.0 \\
\hline
\end{tabular}

Chralce OD-H Column; Hexh-PrOH: $70 / 30 ;$ Flow rate $=0.6 \mathrm{~mL} / \mathrm{min} ; 254 \mathrm{~nm}$;

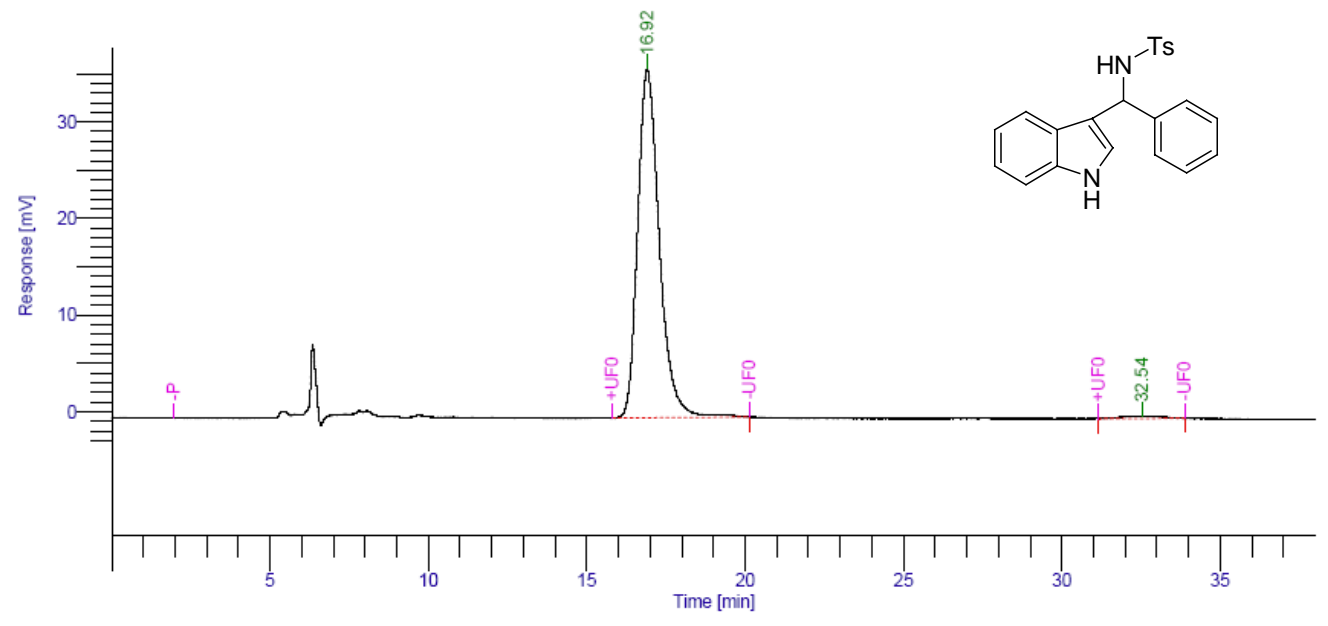

\begin{tabular}{|c|c|c|c|c|c|}
\hline $\begin{array}{c}\text { Peak } \\
\#\end{array}$ & $\begin{array}{l}\text { Time } \\
{[\mathrm{min}]}\end{array}$ & $\begin{array}{c}\text { Area } \\
{\left[\mathrm{uV}^{*} \mathrm{sec}\right]}\end{array}$ & $\begin{array}{l}\text { Height } \\
{[\mathrm{uV}]}\end{array}$ & $\begin{array}{c}\text { Area } \\
{[\%]}\end{array}$ & $\begin{array}{c}\text { Norm. Area } \\
{[\%]}\end{array}$ \\
\hline \multirow{3}{*}{$\begin{array}{l}1 \\
2\end{array}$} & 16.92 & $1.7146 \mathrm{e}+06$ & $3.610 \mathrm{e}+04$ & 98.78 & 98.78 \\
\hline & 32.54 & 21253.7132 & 258.7229 & 1.22 & 1.22 \\
\hline & & $1.7358 \mathrm{e}+06$ & $3.635 \mathrm{e}+04$ & 100.00 & 100.00 \\
\hline
\end{tabular}

Chiralcel OD-H Column; Hex/i-PrOH: 70/30; Flow rate $=0.6 \mathrm{~mL} / \mathrm{min} ; 254 \mathrm{~nm}$; 
$000^{\circ}-$

$+20^{\circ} 0$

$\operatorname{sst} 1$

$0+\varepsilon z$
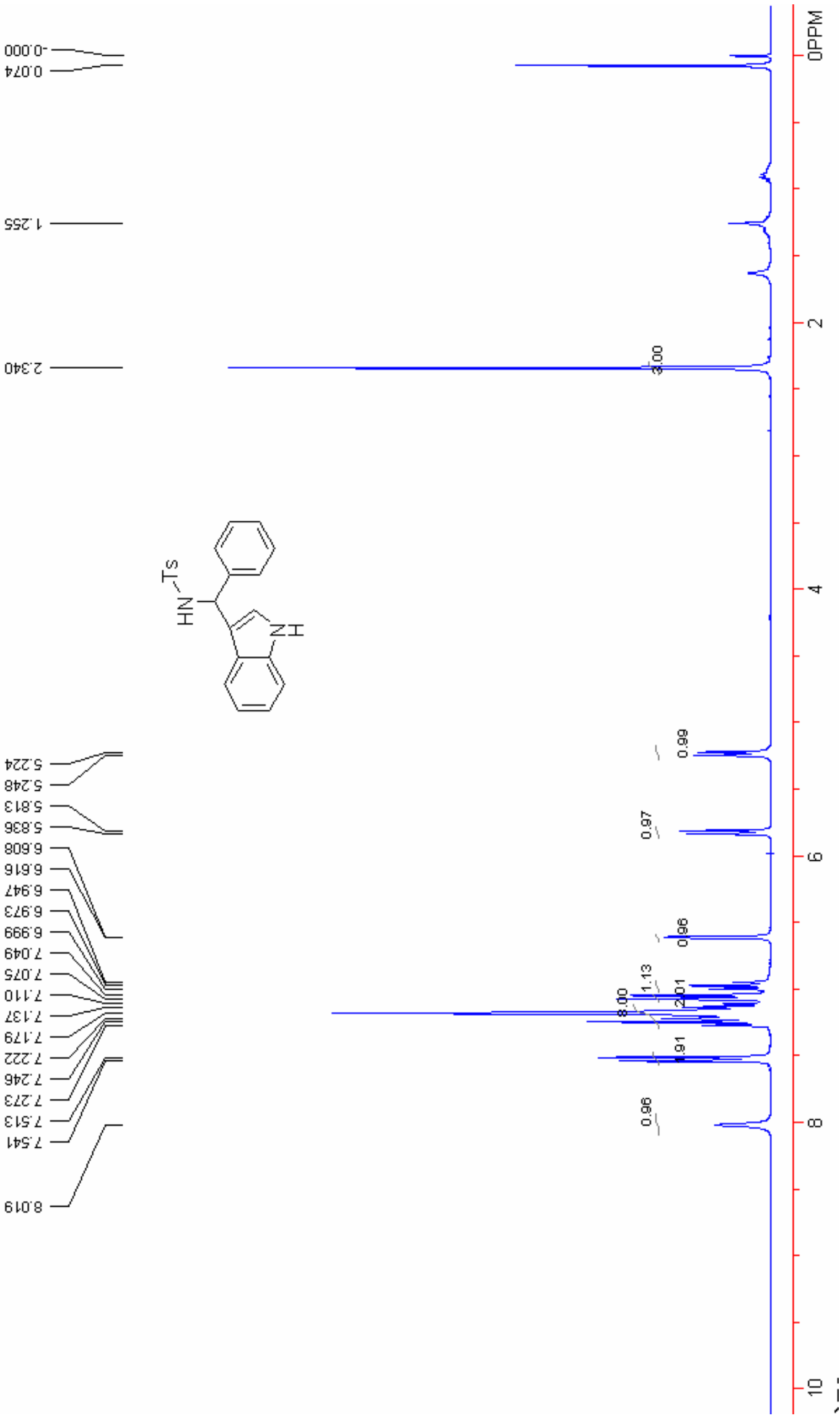

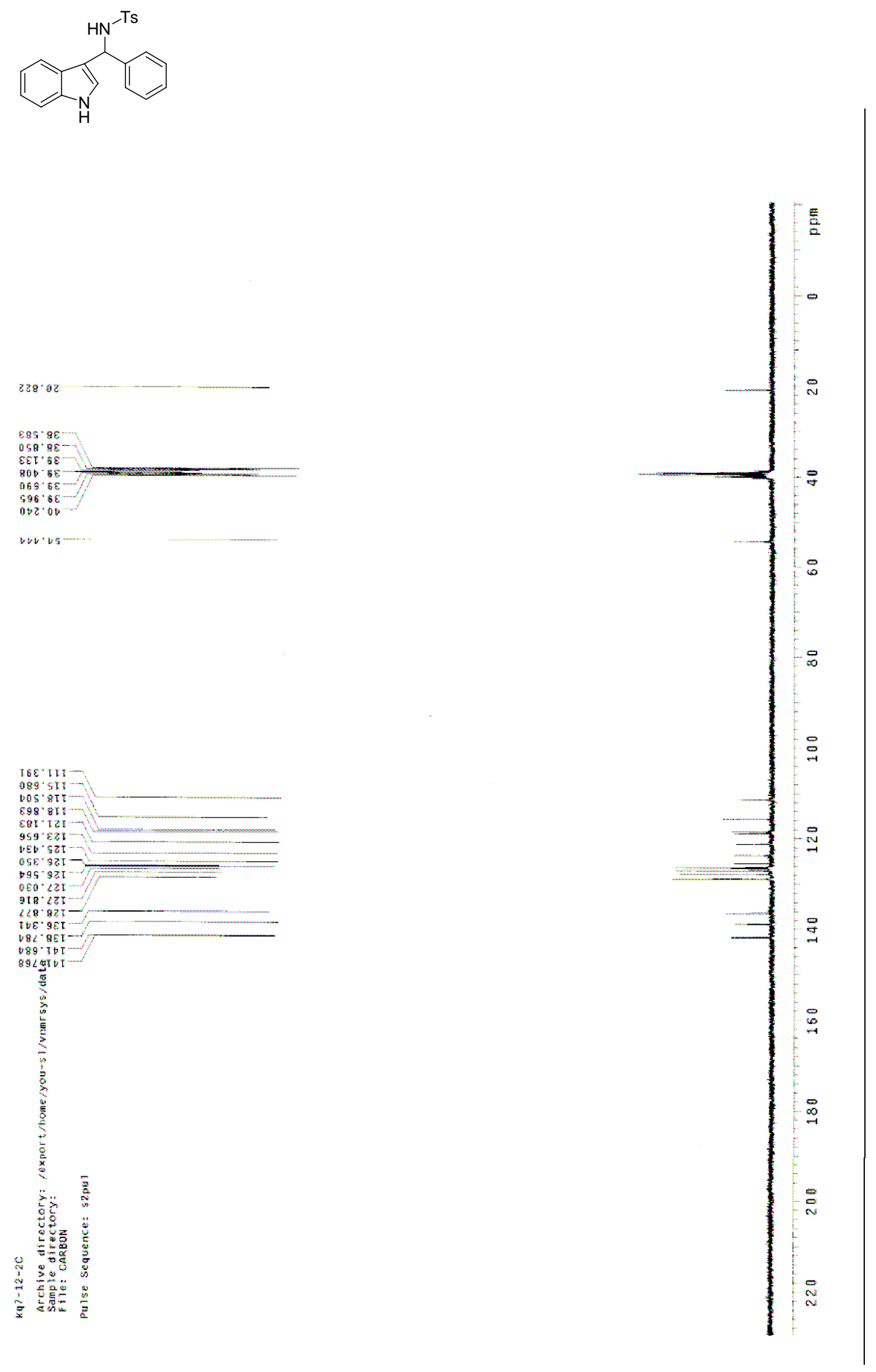


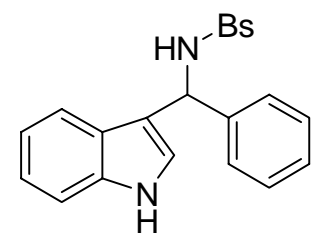

(entry 2, Table 3): $\mathrm{R}_{\mathrm{f}}=0.40$ (ethyl acetate/petroleum ether $=1 / 2, \mathrm{v} / \mathrm{v}$ ); colorless solid, $88 \%$ yield, $99 \%$ ee [Daicel Chiralcel OD-H, Hexanes / IPA $=70 / 30,0.8 \mathrm{ml} \square \mathrm{min}^{-1}, \lambda=254 \mathrm{~nm}, \mathrm{t}$ (major) $=11.96 \mathrm{~min}, \mathrm{t}$ $($ minor $)=21.69 \mathrm{~min}] ;[\alpha]_{\mathrm{D}}{ }^{20}=+18.1^{\circ}(\mathrm{c}=0.77$, Acetone $) .{ }^{1} \mathrm{H}$ NMR $\left(300 \mathrm{MHz}, \mathrm{CDCl}_{3}\right) \delta 5.22(\mathrm{~d}, J=7.2$ $\mathrm{Hz}, 1 \mathrm{H}), 5.88(\mathrm{~d}, J=7.5 \mathrm{~Hz}, 1 \mathrm{H}), 6.62(\mathrm{~d}, J=2.4 \mathrm{~Hz}, 1 \mathrm{H}), 7.00(\mathrm{t}, J=7.5 \mathrm{~Hz}, 1 \mathrm{H}), 7.13-7.31(\mathrm{~m}, 10 \mathrm{H}), 7.43$ $(\mathrm{t}, J=7.5 \mathrm{~Hz}, 1 \mathrm{H}), 7.65(\mathrm{~d}, J=7.2 \mathrm{~Hz}, 2 \mathrm{H}), 8.02(\mathrm{br}, 1 \mathrm{H}) ;{ }^{13} \mathrm{C} \mathrm{NMR}\left(75 \mathrm{MHz}, \mathrm{CDCl}_{3}\right) \delta 55.0,111.3,116.2$, 119.2, 120.0, 122.5, 123.8, 125.3, 127.0, 127.1, 127.4, 128.3, 128.6, 132.2, 136.5, 140.0, 140.4 . 

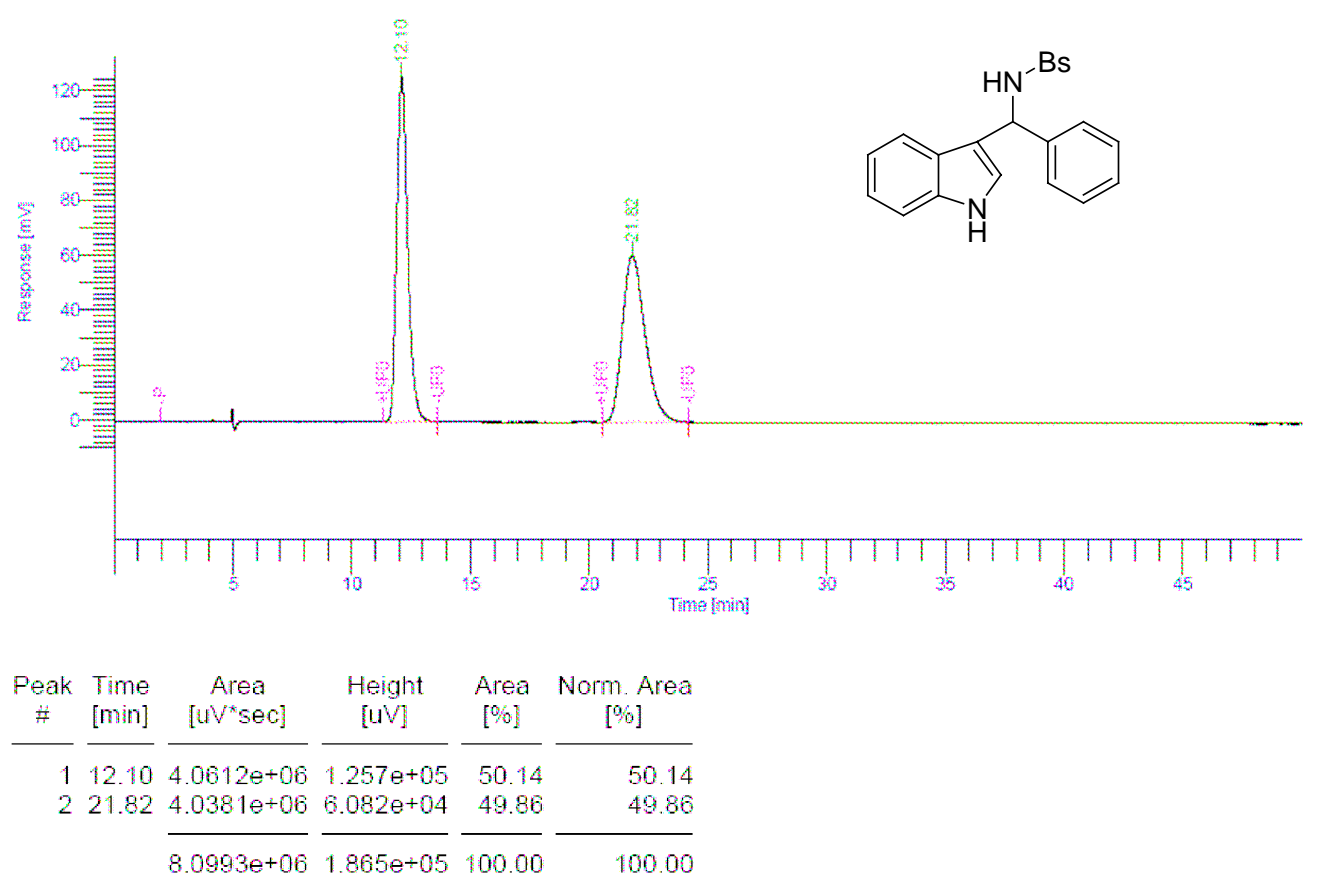

Chiralcel OD-H Column; Hex/1-PrOH: $70 / 30 ;$ Flow rate $=0.8 \mathrm{~mL} / \mathrm{min}, 254 \mathrm{~nm}$;

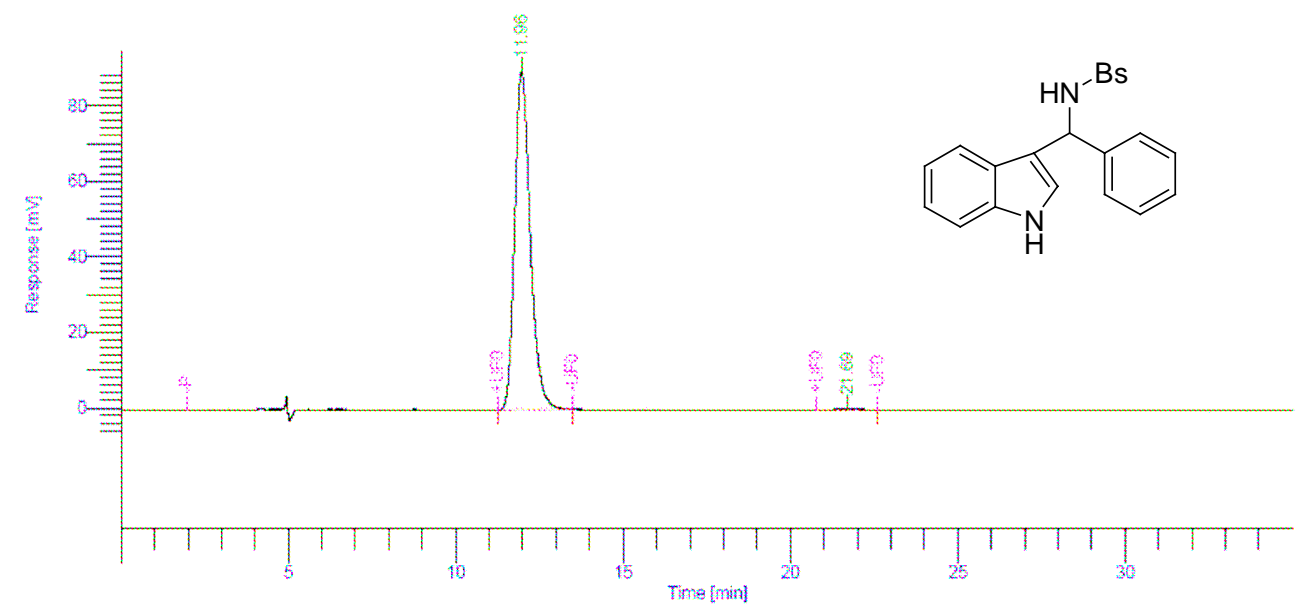

$$
\begin{aligned}
& \text { Peak Time Area Height Area Norm. Area } \\
& { }^{\#} \frac{[\mathrm{min}]}{11.96} \frac{\left[\mathrm{UV}^{*}\right. \text { sec] }}{2.9034 \mathrm{e}+06} \frac{[\mathrm{uV}]}{8.944 \mathrm{e}+04} \frac{[\%]}{99.30} \frac{[\%]}{99.30} \\
& \begin{array}{llllll}
2 & 21.69 & 20517.0250 & 388.9935 & 0.70 & 0.70
\end{array} \\
& \overline{2.9240 e+06} \overline{8.983 e+04} \overline{100.00}-\frac{0.70}{100.00}
\end{aligned}
$$

Chralcel OD-H Column; Hexi-PrOH: 7030; Flow rate $=0.8 \mathrm{~mL} / \mathrm{min} ; 254 \mathrm{~nm}$; 


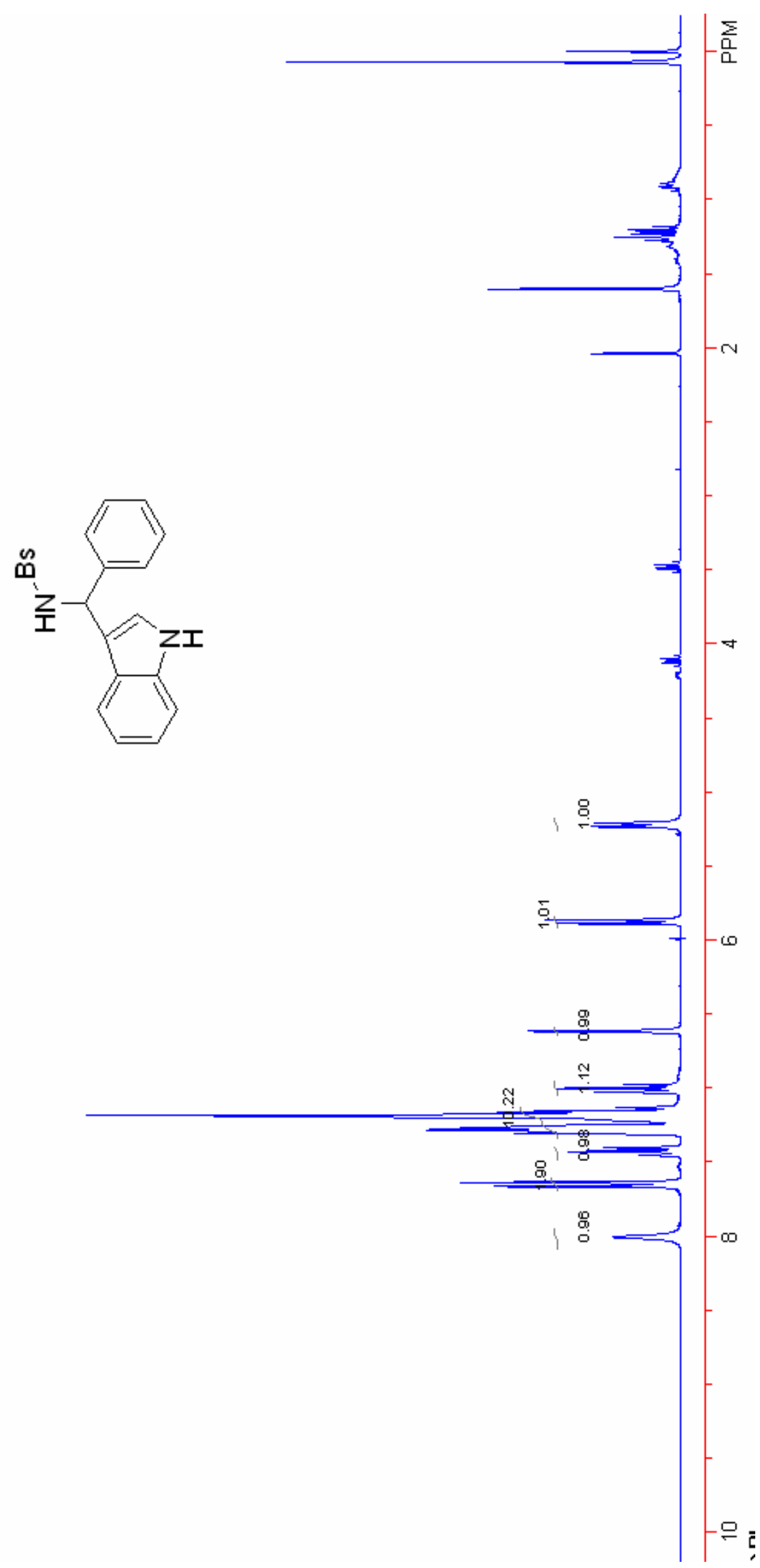




$$
i
$$


<smiles>COc1ccc2[nH]cc(C(NC(F)(F)F)c3ccccc3)c2c1</smiles>

(entry 3, Table 3): $\mathrm{R}_{\mathrm{f}}=0.40$ (ethyl acetate/petroleum ether $=1 / 2$, v/v); colorless solid, $87 \%$ yield, $97 \%$ ee [Daicel Chiralcel OD-H, Hexanes / IPA $=60 / 40,0.6 \mathrm{ml} \square \mathrm{min}^{-1}, \lambda=254 \mathrm{~nm}, \mathrm{t}$ (major) $=9.10 \mathrm{~min}, \mathrm{t}$ $($ minor $)=26.94 \mathrm{~min}] ;[\alpha]_{\mathrm{D}}{ }^{20}=+25.4^{\mathrm{o}}(\mathrm{c}=0.50$, Acetone $) .{ }^{1} \mathrm{H}$ NMR $\left(300 \mathrm{MHz}, \mathrm{CDCl}_{3}\right) \delta 2.24(\mathrm{~s}, 3 \mathrm{H})$, $3.63(\mathrm{~s}, 3 \mathrm{H}), 5.56(\mathrm{~d}, J=7.5 \mathrm{~Hz}, 1 \mathrm{H}), 5.73(\mathrm{~d}, J=7.5 \mathrm{~Hz}, 1 \mathrm{H}), 6.44(\mathrm{~d}, J=1.8 \mathrm{~Hz}, 1 \mathrm{H}), 6.69-6.73(\mathrm{~m}, 2 \mathrm{H})$, $6.94(\mathrm{~d}, J=8.1 \mathrm{~Hz}, 2 \mathrm{H}), 7.03-7.14(\mathrm{~m}, 6 \mathrm{H}), 7.44(\mathrm{~d}, J=7.8 \mathrm{~Hz}, 2 \mathrm{H}), 8.18(\mathrm{br}, 1 \mathrm{H}) ;{ }^{13} \mathrm{C} \mathrm{NMR}(75 \mathrm{MHz}$, $\left.\mathrm{CDCl}_{3}\right) \delta 21.3,54.9,55.6,100.8,112.0,112.4,115.5,124.6,125.8,126.9,127.0,127.1,128.1,129.1$, 131.5, 137.3, 140.1, 142.8, 153.7; MS (EI): m/z (\%relative intensity) 406 (M+, 4), 91 (100), 171 (55), 235 (51), 155 (48), 107 (23). HRMS Calcd for $\left(\mathrm{M}^{+}\right) \mathrm{C}_{23} \mathrm{H}_{22} \mathrm{~N}_{2} \mathrm{O}_{3} \mathrm{~S}$ : 406.1351. Found: 406.1346. 


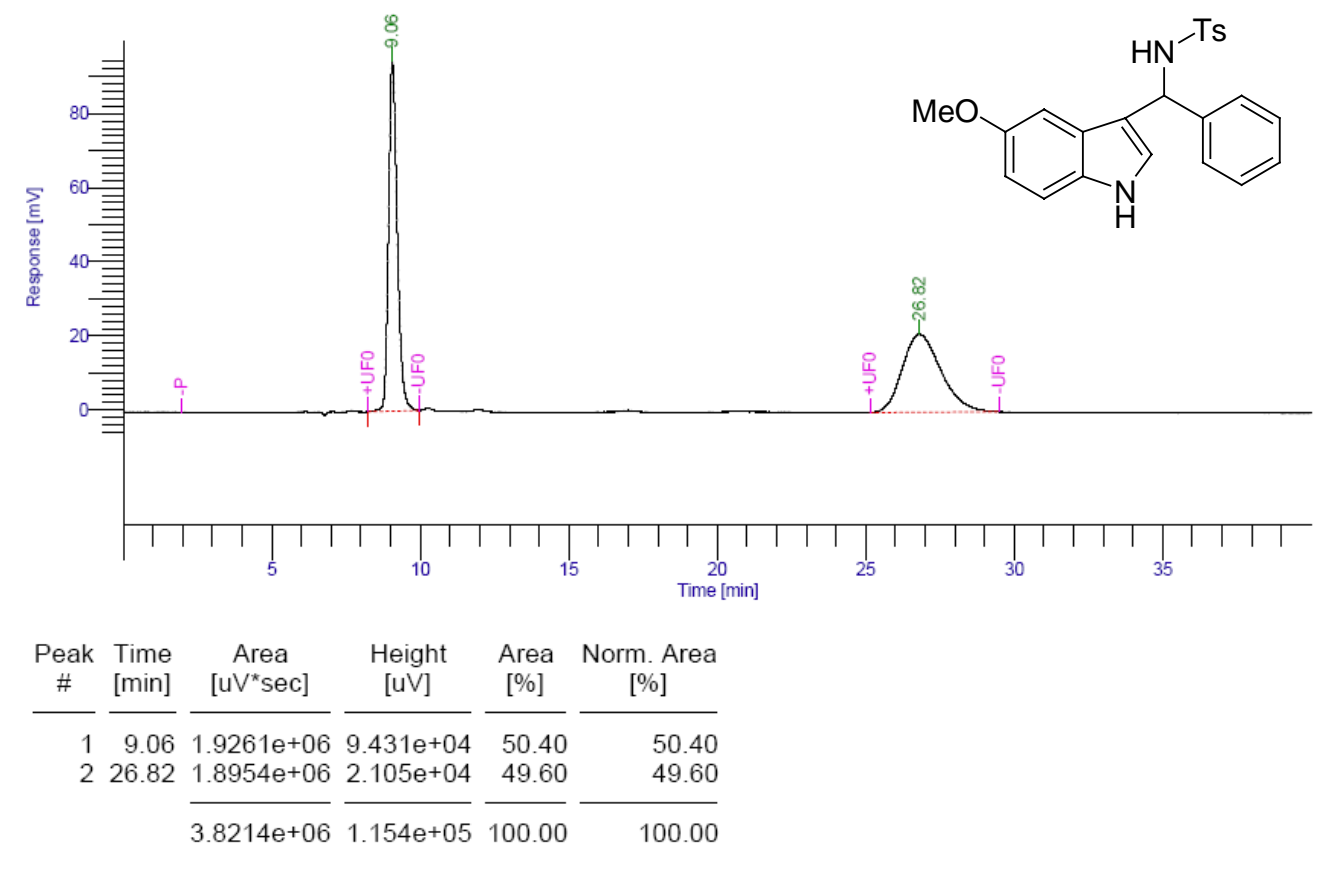

Chiralcel OD-H Column; Hex/i-PrOH: 60/40; Flow rate $=0.6 \mathrm{~mL} / \mathrm{min} ; 254$ nm;

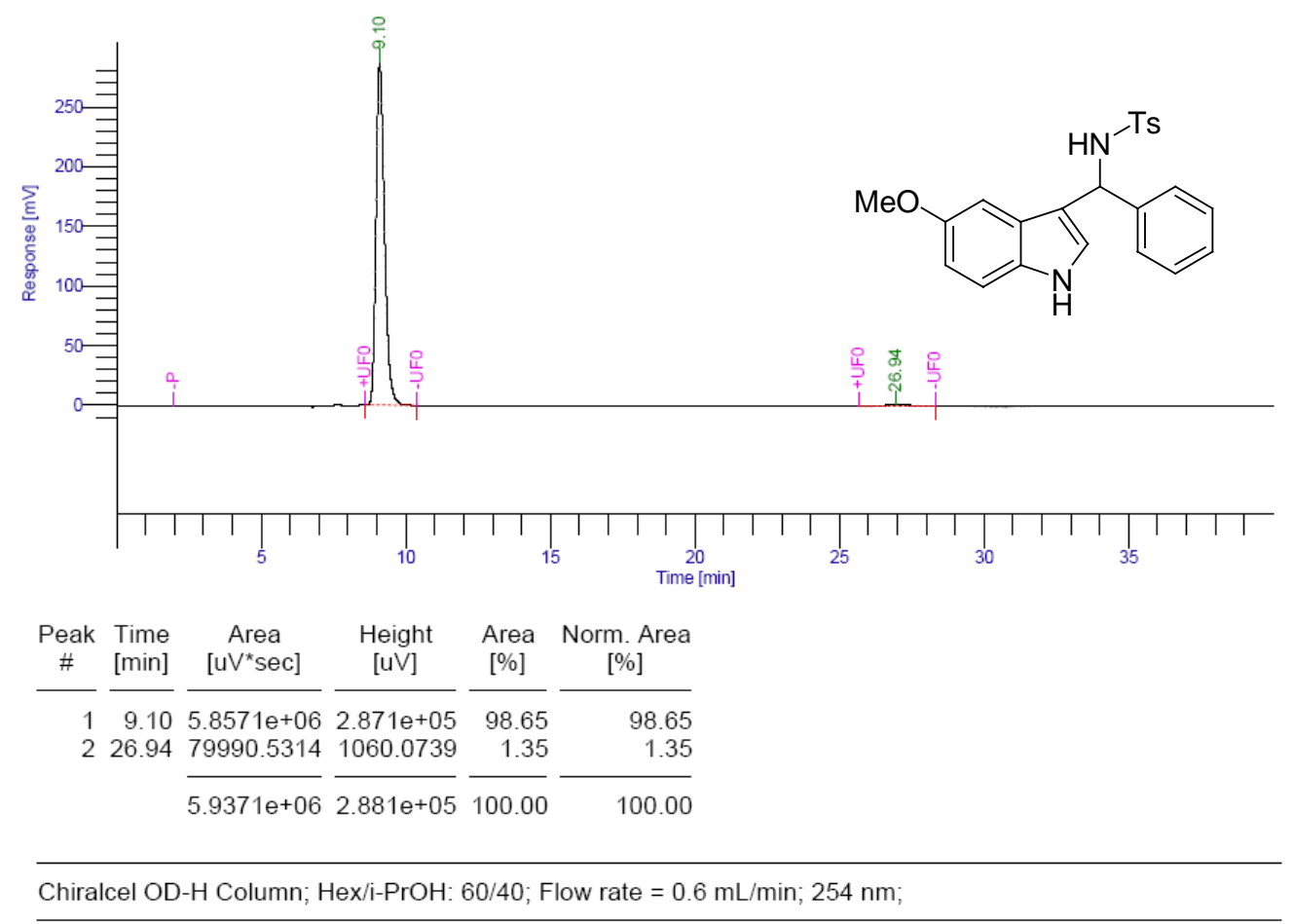


$ฮ \tau เ$

$z t z z$

$90 L z$

$9 z 9 \varepsilon-$

$6 t s$.

$t \angle S S \longrightarrow$

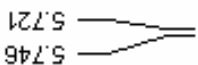

$902 \mathrm{~S}$

9079

$8699 \longrightarrow$

$0029-2$

$8 \subset L 9-1$

$\angle Z 69$ -

$\mathrm{tS6} 9 \longrightarrow$

$\mathrm{DSO}_{\mathrm{SO}} \mathrm{L}$

$9602-2=$

$S E L L$

$S Z+2-$

ISt 2 -

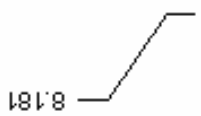

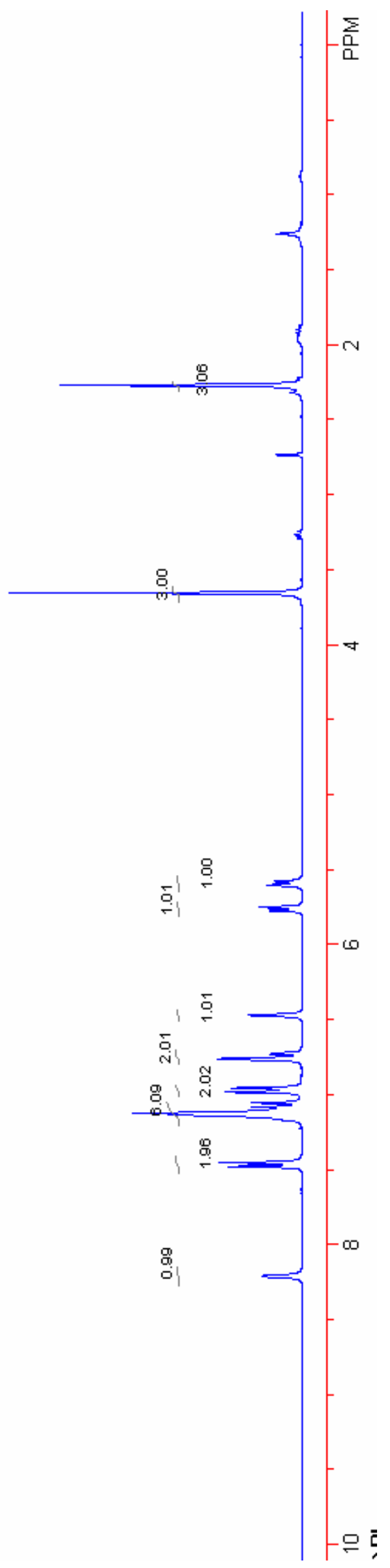



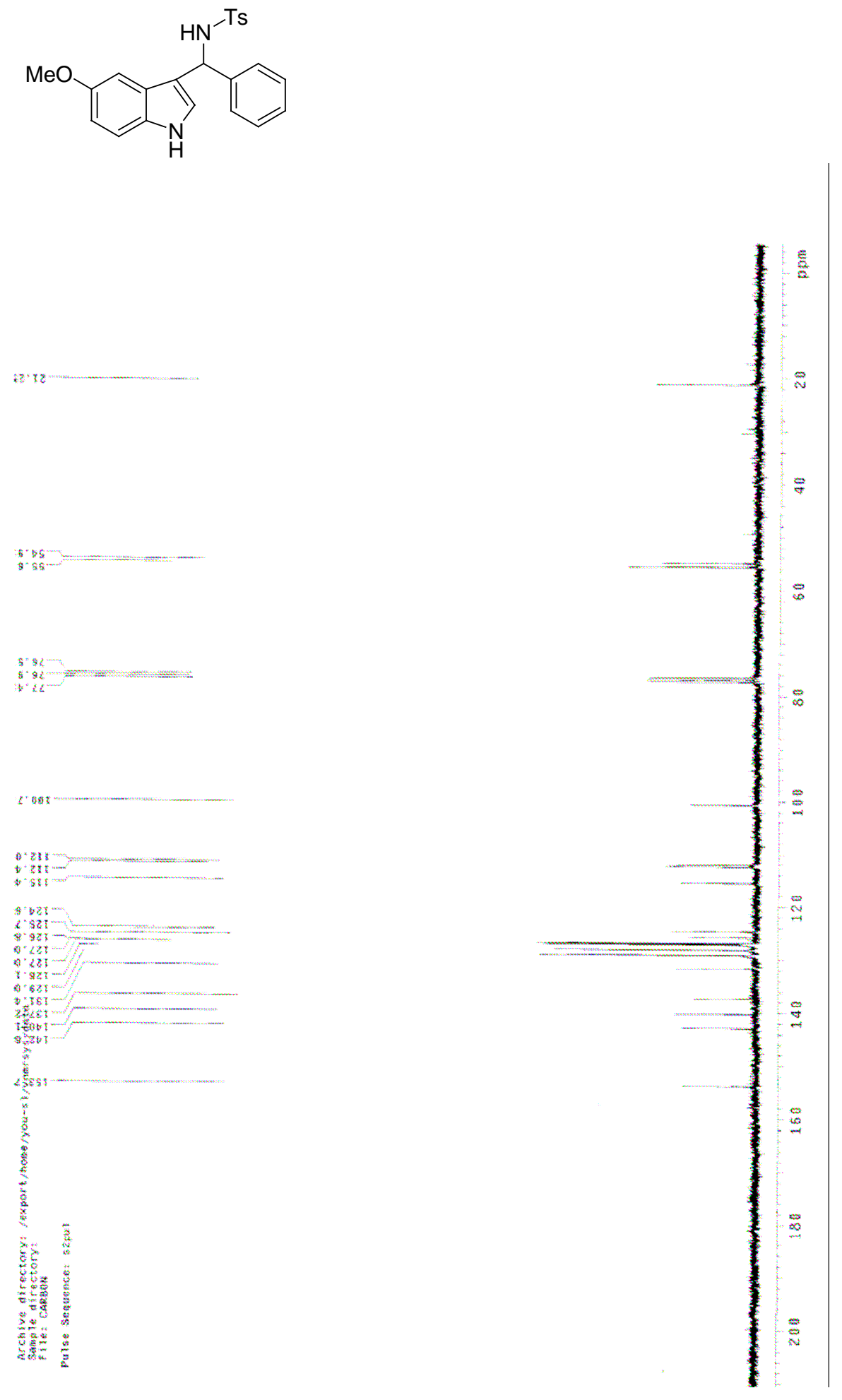


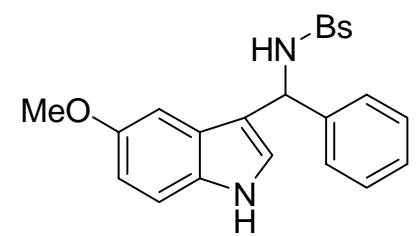

(entry 4, Table 3): $\mathrm{R}_{\mathrm{f}}=0.30$ (ethyl acetate/petroleum ether $=1 / 2, \mathrm{v} / \mathrm{v}$ ); colorless solid, $84 \%$ yield, $99 \%$ ee [Daicel Chiralcel OD-H, Hexanes / IPA $=70 / 30,0.8 \mathrm{ml} \square \min ^{-1}, \lambda=254 \mathrm{~nm}, \mathrm{t}$ (major) $=8.92 \mathrm{~min}, \mathrm{t}$ $($ minor $)=33.31 \mathrm{~min}] ;[\alpha]_{\mathrm{D}}{ }^{20}=+23.0^{\circ}(\mathrm{c}=0.97$, Acetone $) .{ }^{1} \mathrm{H} \mathrm{NMR}\left(300 \mathrm{MHz}, \mathrm{CDCl}_{3}\right) \delta 3.71(\mathrm{~s}, 3 \mathrm{H})$, $5.44(\mathrm{~d}, J=7.2 \mathrm{~Hz}, 1 \mathrm{H}), 5.84(\mathrm{~d}, J=7.5 \mathrm{~Hz}, 1 \mathrm{H}), 6.52(\mathrm{~d}, J=2.7 \mathrm{~Hz}, 1 \mathrm{H}), 6.79\left(\mathrm{dd}, J_{1}=2.4 \mathrm{~Hz}, J_{2}=9.0\right.$ $\mathrm{Hz}, 1 \mathrm{H}), 6.85(\mathrm{~d}, J=1.8 \mathrm{~Hz}, 1 \mathrm{H}), 7.12-7.25(\mathrm{~m}, 8 \mathrm{H}), 7.38$ (t, $J=7.5 \mathrm{~Hz}, 1 \mathrm{H}), 7.62(\mathrm{~d}, J=8.1 \mathrm{~Hz}, 2 \mathrm{H}), 7.96$ (br, $1 \mathrm{H}) ;{ }^{13} \mathrm{C}$ NMR $\left(75 \mathrm{MHz}, \mathrm{CDCl}_{3}\right) \delta 55.1,55.8,76.6,77.0,77.4,100.9,109.7,112.0,115.8,124.5$, 125.8, 126.9, 127.1, 127.4, 128.3, 128.6, 131.5, 132.1, 139.9, 154.1; MS (EI): m/z (\%relative intensity) $392\left(\mathrm{M}^{+}, 2\right), 235$ (100), 77 (52), 165 (37), 220 (37), 93 (18). HRMS Calcd for $\left(\mathrm{M}^{+}\right) \mathrm{C}_{22} \mathrm{H}_{20} \mathrm{~N}_{2} \mathrm{O}_{3} \mathrm{~S}$ : 392.1201. Found: 392.1202. 


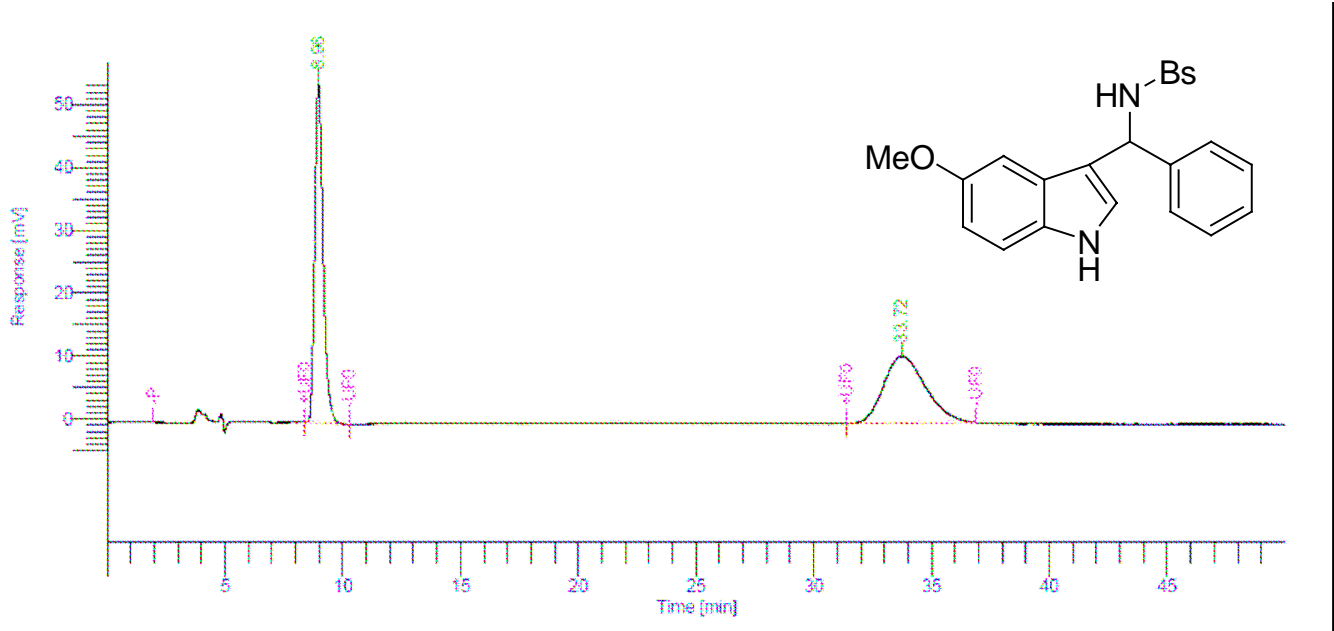

\begin{tabular}{|c|c|c|c|c|c|}
\hline $\begin{array}{c}\text { Peak } \\
\#\end{array}$ & $\begin{array}{l}\text { Time } \\
\text { [min] }\end{array}$ & $\begin{array}{c}\text { Area } \\
{\left[\mathrm{uV}^{*} \mathrm{sec}\right]}\end{array}$ & $\begin{array}{l}\text { Height } \\
\text { [uV] }\end{array}$ & $\begin{array}{c}\text { Area } \\
{[\%]}\end{array}$ & $\begin{array}{c}\text { Norm. Area } \\
{[\%]}\end{array}$ \\
\hline 1 & 8.96 & $1.3209 e+06$ & $5.405 e+04$ & 50.10 & 50.10 \\
\hline 2 & 33.72 & $1.3157 e+06$ & $1.067 e+04$ & 49.90 & 49.90 \\
\hline
\end{tabular}

Chiralcel OD-H Column; Hex/i-PrOH: 70/30; Flow rate $=0.8 \mathrm{~mL} / \mathrm{min} ; 254 \mathrm{~nm}$;

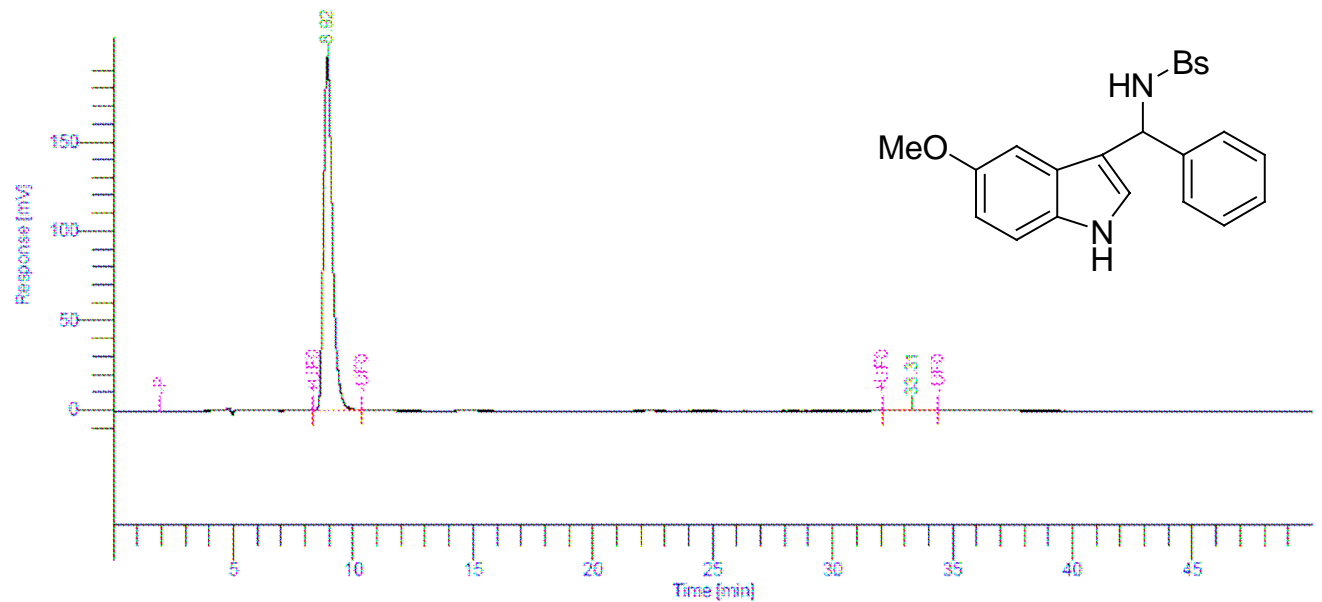

\begin{tabular}{|c|c|c|c|c|c|}
\hline $\begin{array}{c}\text { Peak } \\
\text { \# }\end{array}$ & $\begin{array}{l}\text { Time } \\
\text { [min] }\end{array}$ & $\begin{array}{c}\text { Area } \\
{\left[\mathrm{V}^{*} \text { sec] }\right.}\end{array}$ & $\begin{array}{c}\text { Height } \\
\text { [uV] }\end{array}$ & $\begin{array}{c}\text { Area } \\
{[\%]}\end{array}$ & $\begin{array}{c}\text { Norm Area } \\
{[\%]}\end{array}$ \\
\hline \multirow{3}{*}{$\begin{array}{l}1 \\
2\end{array}$} & 8.92 & $4.9938 e+06$ & $1.972 e+05$ & 99.39 & 99.39 \\
\hline & 33.31 & 30784.5053 & 412.8950 & 0.61 & 0.61 \\
\hline & & $5.0246 e+06$ & $1.977 e+05$ & 100.00 & 100.00 \\
\hline
\end{tabular}

Chiralcel OD-H Column; Hexi-PrOH: $70 / 30$; Flow rate $=0.8 \mathrm{~mL} / \mathrm{min} ; 254 \mathrm{~nm}$ 
$0000-$

$+200 \div$

SSZ !

$\angle 0 \angle \varepsilon$

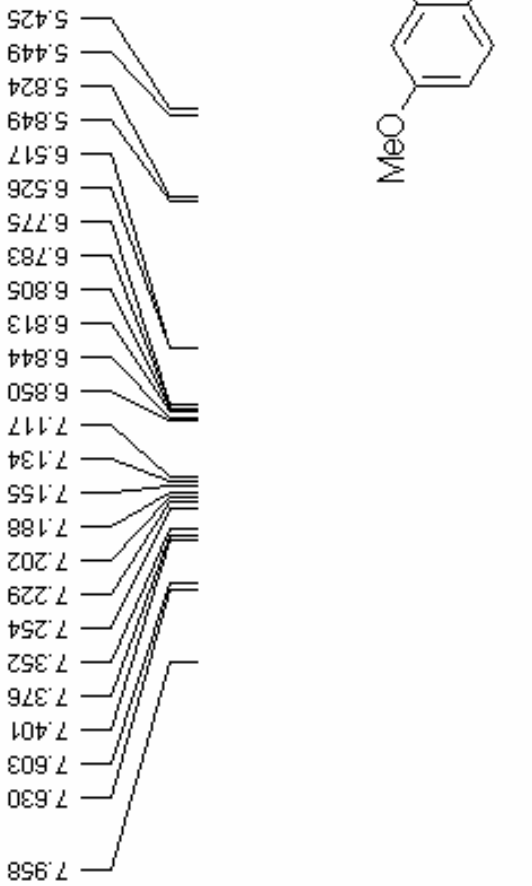

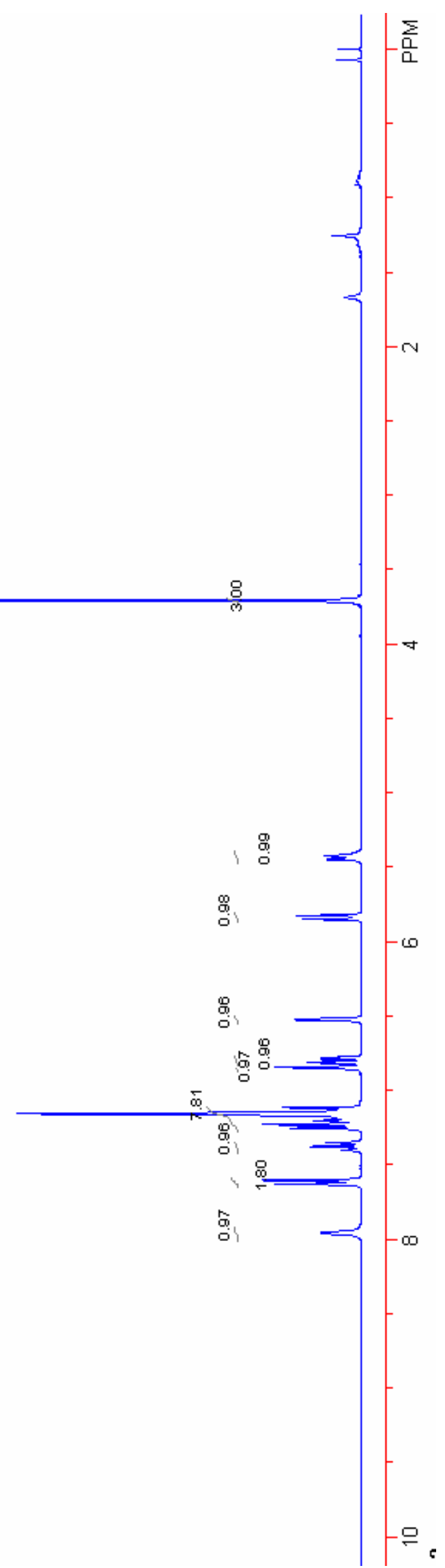




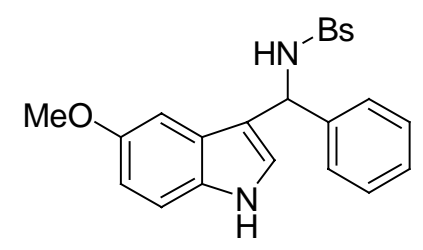

Art 5 s
85255

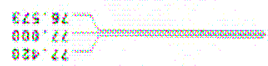

200.098

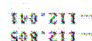

Sag 211 -

806 b 21

beg. $5 \geq 3-$

105

-

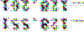

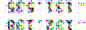

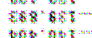
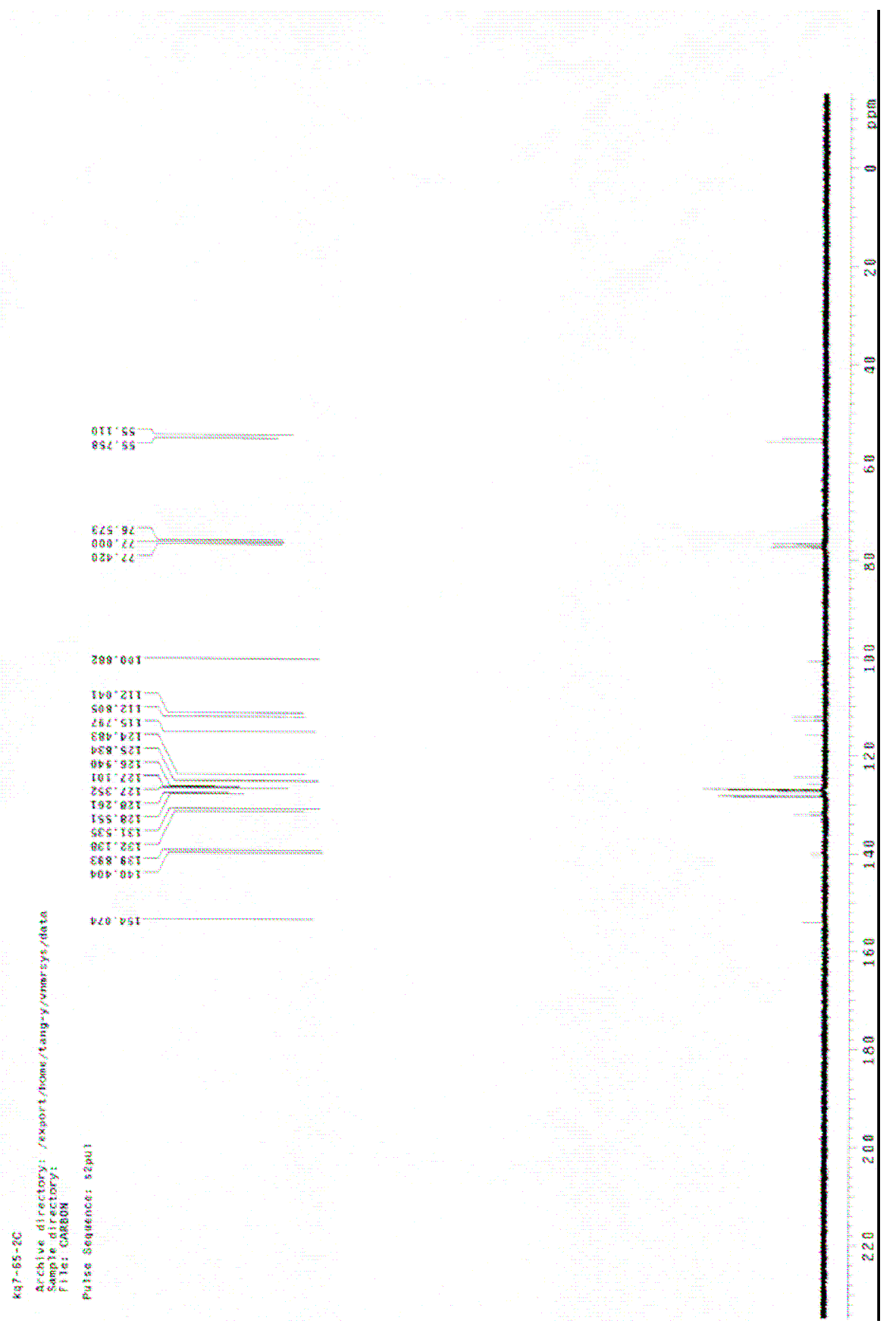


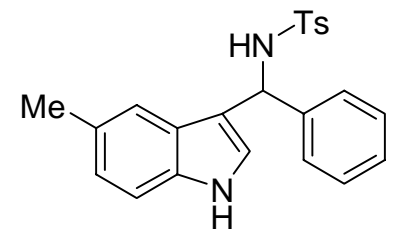

(entry 5, Table 3): $\mathrm{R}_{\mathrm{f}}=0.30$ (ethyl acetate/petroleum ether $=1 / 3$, v/v); colorless solid, $89 \%$ yield,

$>99 \%$ ee [Daicel Chiralcel OD-H, Hexanes $/ \mathrm{IPA}=70 / 30,0.8 \mathrm{ml} \square \mathrm{min}^{-1}, \lambda=254 \mathrm{~nm}, \mathrm{t}$ (major) $=8.41 \mathrm{~min}$, $\mathrm{t}($ minor $)=22.25 \mathrm{~min}] ;[\alpha]_{\mathrm{D}}{ }^{20}=+21.4^{\mathrm{o}}\left(\mathrm{c}=1.0\right.$, Acetone). ${ }^{1} \mathrm{H}$ NMR $\left(300 \mathrm{MHz}, \mathrm{CDCl}_{3}\right) \delta 2.28(\mathrm{~s}, 3 \mathrm{H}), 2.34$ $(\mathrm{s}, 3 \mathrm{H}), 5.22(\mathrm{~d}, J=6.6 \mathrm{~Hz}, 1 \mathrm{H}), 5.74(\mathrm{~d}, J=6.6 \mathrm{~Hz}, 1 \mathrm{H}), 6.43(\mathrm{~s}, 1 \mathrm{H}), 6.85(\mathrm{~s}, 1 \mathrm{H}), 6.92(\mathrm{~d}, J=8.7 \mathrm{~Hz}, 1 \mathrm{H})$, 7.06-7.10 (m, 3H), 7.16-7.20 (m, 5H), $7.53(\mathrm{~d}, J=7.8 \mathrm{~Hz}, 2 \mathrm{H}), 7.95(\mathrm{br}, 1 \mathrm{H}) ;{ }^{13} \mathrm{C} \mathrm{NMR}\left(75 \mathrm{MHz}, \mathrm{CDCl}_{3}\right)$ $\delta 21.3,21.4,54.7,111.0,115.5,118.4,123.9,124.2,125.5,127.0,127.1,128.2,128.9,129.2,134.7,137.2$, 140.4, 143.0. 


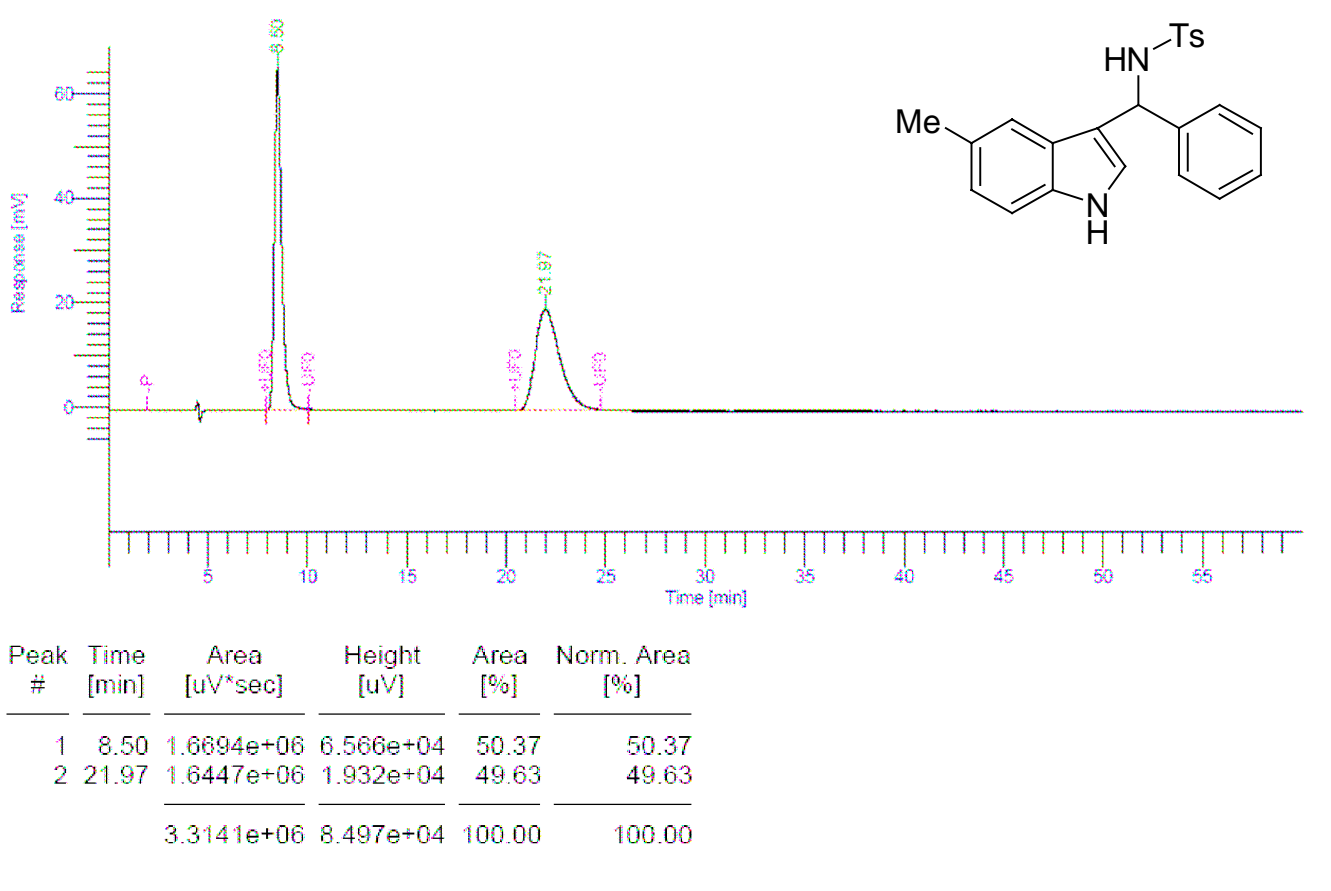

Chiralcel OD-H Column; Hexi-PrOH: $70 / 30$; Flow rate $=0.8 \mathrm{~mL} / \mathrm{min} ; 254 \mathrm{~nm}$;

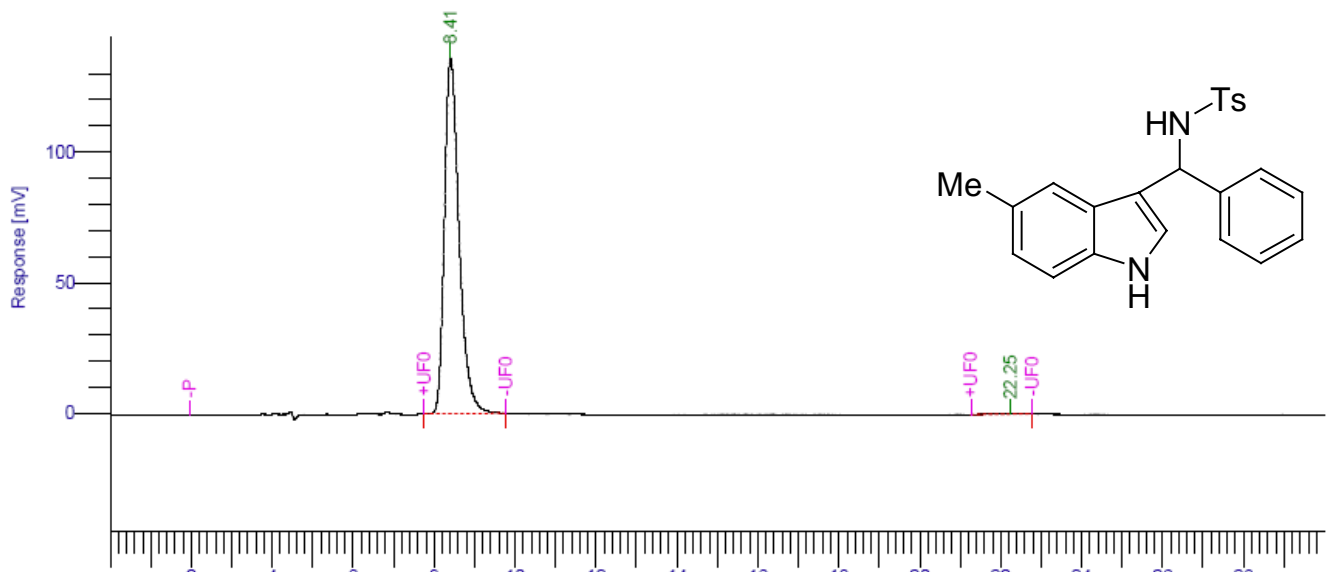

\begin{tabular}{|c|c|c|c|c|c|}
\hline $\begin{array}{c}\text { Peak } \\
\stackrel{\#}{\#}\end{array}$ & $\begin{array}{l}\text { Time } \\
\text { [min] }\end{array}$ & $\begin{array}{c}\text { Area } \\
{\left[\mathrm{uV}^{*} \mathrm{sec}\right]}\end{array}$ & $\begin{array}{l}\text { Height } \\
{[\mathrm{uV}]}\end{array}$ & $\begin{array}{c}\text { Area } \\
{[\%]}\end{array}$ & $\begin{array}{c}\text { Norm. Area } \\
{[\%]}\end{array}$ \\
\hline & 8.41 & 3.4 & 1.36 & 996 & 99.68 \\
\hline & 22.2 & 10 & 24 & 0.32 & 0.32 \\
\hline & & & 1.362 & 100.00 & 00. \\
\hline
\end{tabular}

Chiralcel OD-H Column; Hexi-PrOH: $70 / 30$, Flow rate $=0.8 \mathrm{~mL} / \mathrm{min} ; 254 \mathrm{~nm}$ 


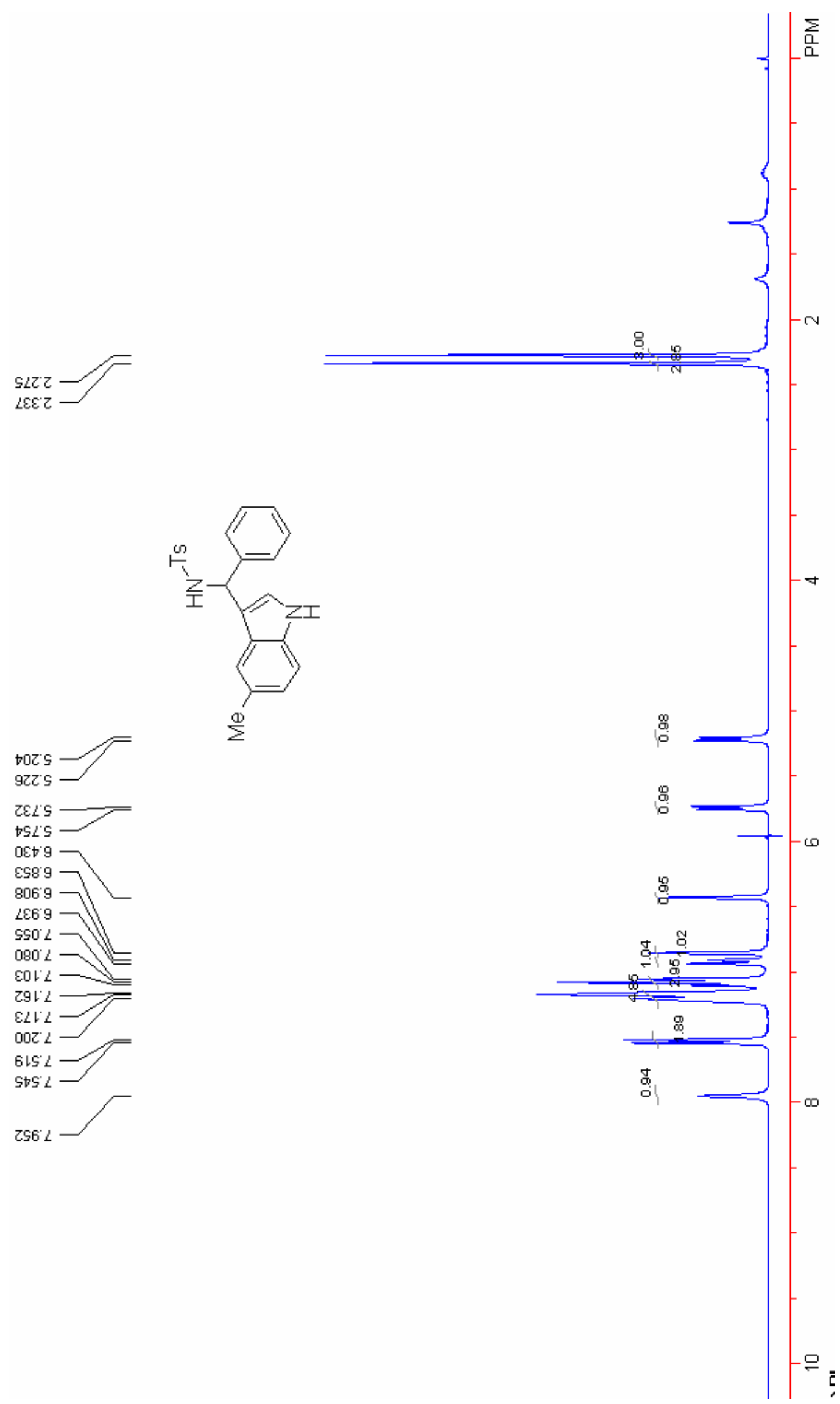


<smiles>Cc1ccc2[nH]cc(C(NC(F)(F)F)c3ccccc3)c2c1</smiles>

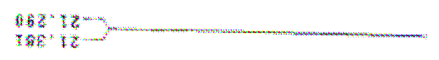

$\operatorname{sis} 2 a$

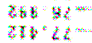

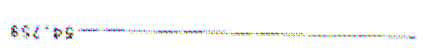

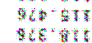

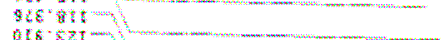

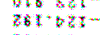

$395-53$

$864+28 I-2=$

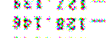

208. 921

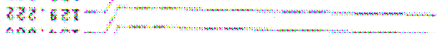

492 .

abs at

she 307
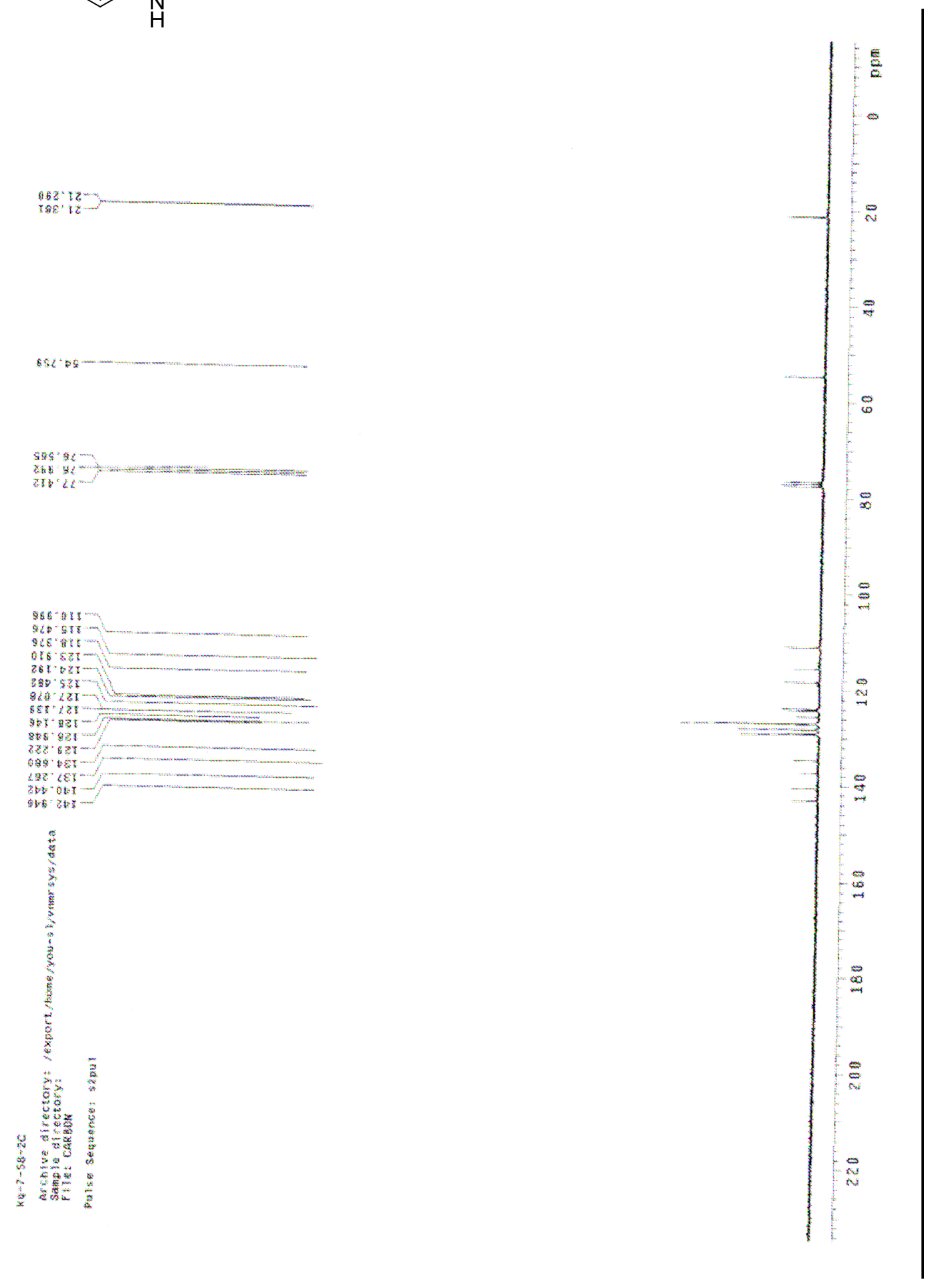
<smiles>Cc1ccc2[nH]cc(C(NC(=O)c3ccccc3)c3ccccc3)c2c1</smiles>

(entry 6, Table 3): $\mathrm{R}_{\mathrm{f}}=0.40$ (ethyl acetate/petroleum ether $=1 / 2$, v/v); colorless solid, $83 \%$ yield, $99 \%$ ee [Daicel Chiralcel OD-H, Hexanes / IPA $=70 / 30,0.8 \mathrm{ml} \square \min ^{-1}, \lambda=254 \mathrm{~nm}, \mathrm{t}$ (major) = $9.46 \mathrm{~min}, \mathrm{t}$ $($ minor $)=24.11 \mathrm{~min}] ;[\alpha]_{\mathrm{D}}{ }^{20}=+20.2^{\circ}(\mathrm{c}=1.0$, Acetone $) .{ }^{1} \mathrm{H}$ NMR $\left(300 \mathrm{MHz}, \mathrm{CDCl}_{3}\right) \delta 2.31(\mathrm{~s}, 3 \mathrm{H}), 5.22$ $(\mathrm{d}, J=6.9 \mathrm{~Hz}, 1 \mathrm{H}), 5.81(\mathrm{~d}, J=6.9 \mathrm{~Hz}, 1 \mathrm{H}), 6.47(\mathrm{~d}, J=2.4 \mathrm{~Hz}, 1 \mathrm{H}), 6.95-6.97(\mathrm{~m}, 2 \mathrm{H}), 7.12-7.24(\mathrm{~m}, 6 \mathrm{H})$, $7.30(\mathrm{t}, J=7.8 \mathrm{~Hz}, 2 \mathrm{H}), 7.44(\mathrm{t}, J=7.2 \mathrm{~Hz}, 1 \mathrm{H}), 7.67(\mathrm{~d}, J=7.2 \mathrm{~Hz}, 2 \mathrm{H}), 7.90(\mathrm{br}, 1 \mathrm{H}) ;{ }^{13} \mathrm{C} \mathrm{NMR}(75 \mathrm{MHz}$, $\left.\mathrm{CDCl}_{3}\right) \delta 21.4,54.9,110.9,115.6,118.5,124.1,125.5,127.0,127.1,127.3,128.2,128.6,129.2,132.2$, 134.7, 140.2, 140.3; MS (EI): m/z (\%relative intensity) 376 (M+1 8), 219 (100), 77 (50), 204 (44), 157 (26). HRMS Calcd for $\left(\mathrm{M}^{+}\right) \mathrm{C}_{22} \mathrm{H}_{20} \mathrm{~N}_{2} \mathrm{O}_{2} \mathrm{~S}: 376.1245$. Found: 376.1264 . 


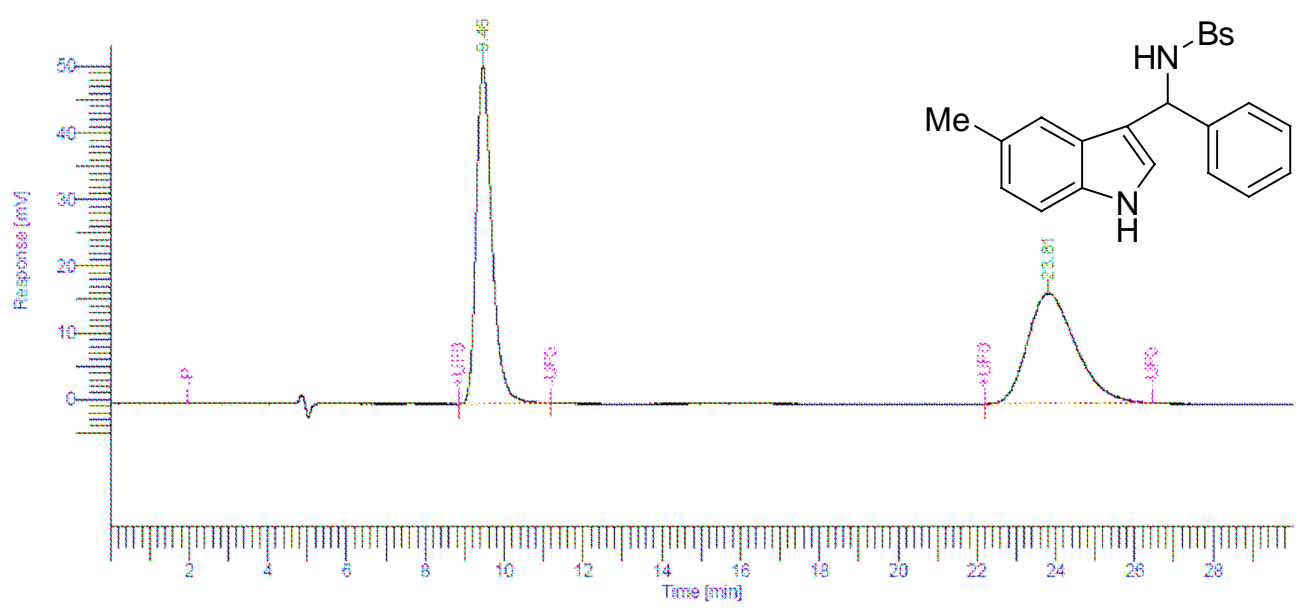

\begin{tabular}{|c|c|c|c|c|c|}
\hline $\begin{array}{c}\text { Peak } \\
\#\end{array}$ & $\begin{array}{l}\text { Time } \\
\text { [min] }\end{array}$ & $\begin{array}{c}\text { Area } \\
\text { [uV }{ }^{*} \text { sec] }\end{array}$ & $\begin{array}{l}\text { Height } \\
\text { [uV] }\end{array}$ & $\begin{array}{c}\text { Area } \\
{[\%]}\end{array}$ & $\begin{array}{c}\text { Nom. Area } \\
{[\%]}\end{array}$ \\
\hline \multirow{3}{*}{$\begin{array}{l}1 \\
2\end{array}$} & 9.46 & $1.4073 e+06$ & $5.071 e+04$ & 50.13 & 50.13 \\
\hline & 23.81 & $1.4003 e+06$ & $1.655 \mathrm{e}+04$ & 49.87 & 49.87 \\
\hline & & $2.8076 e+06$ & $6.726 e+04$ & 100.00 & 100.0 \\
\hline
\end{tabular}

Chiralcel OD-H Column; Hex/i-PrOH: $70 / 30$; Flow rate $=0.8 \mathrm{~mL} / \mathrm{min} ; 254 \mathrm{~nm}$;

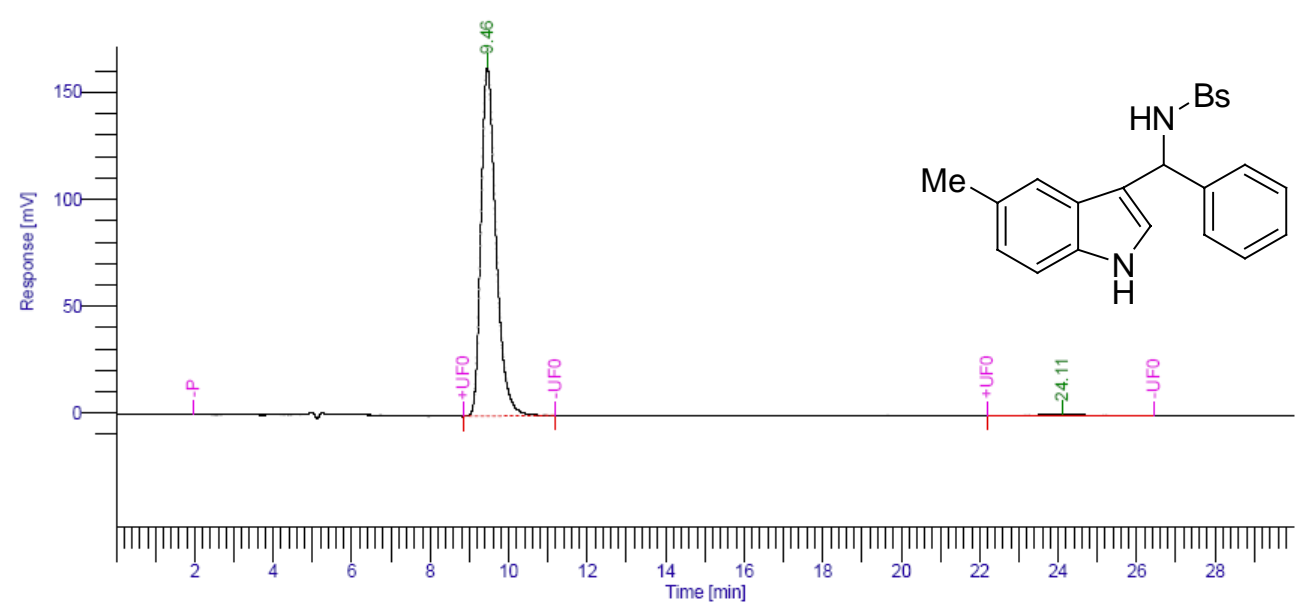

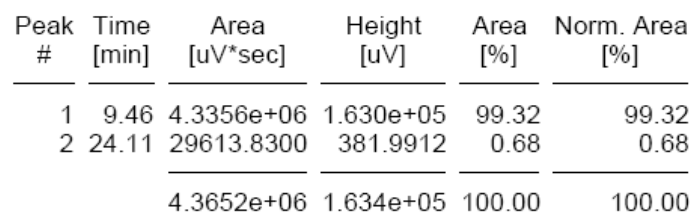

Chiralcel OD-H Column; Hex/i-PrOH: 70/30; Flow rate $=0.8 \mathrm{~mL} / \mathrm{min} ; 254 \mathrm{~nm}$; 
$0\llcorner\varepsilon z$

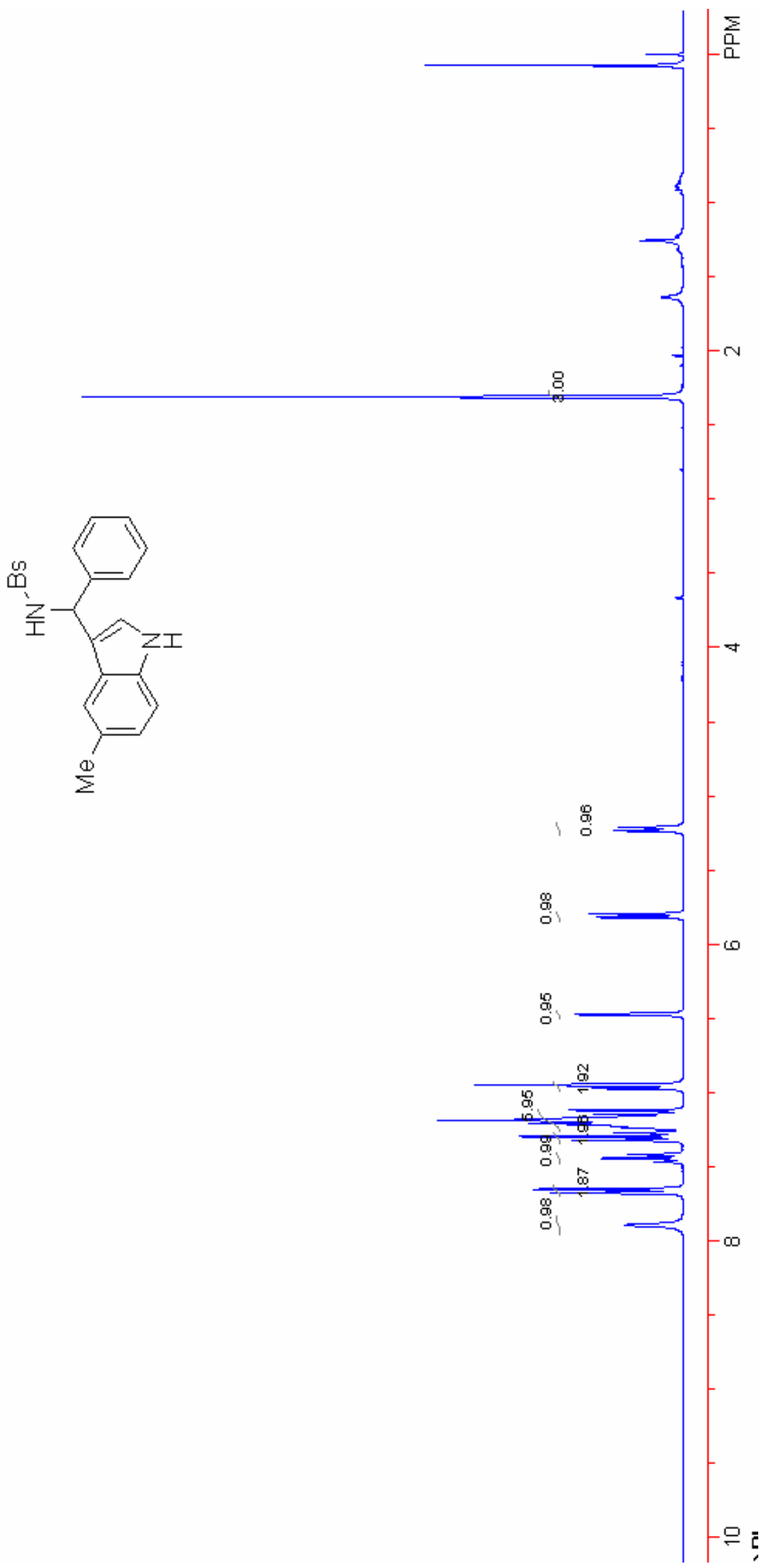


<smiles>Cc1ccc2[nH]cc(C(NC(=O)c3ccccc3)c3ccccc3)c2c1</smiles>

$98 \% \cdot 12-1$

30635

ELS: $92^{-m m}$

oet 2
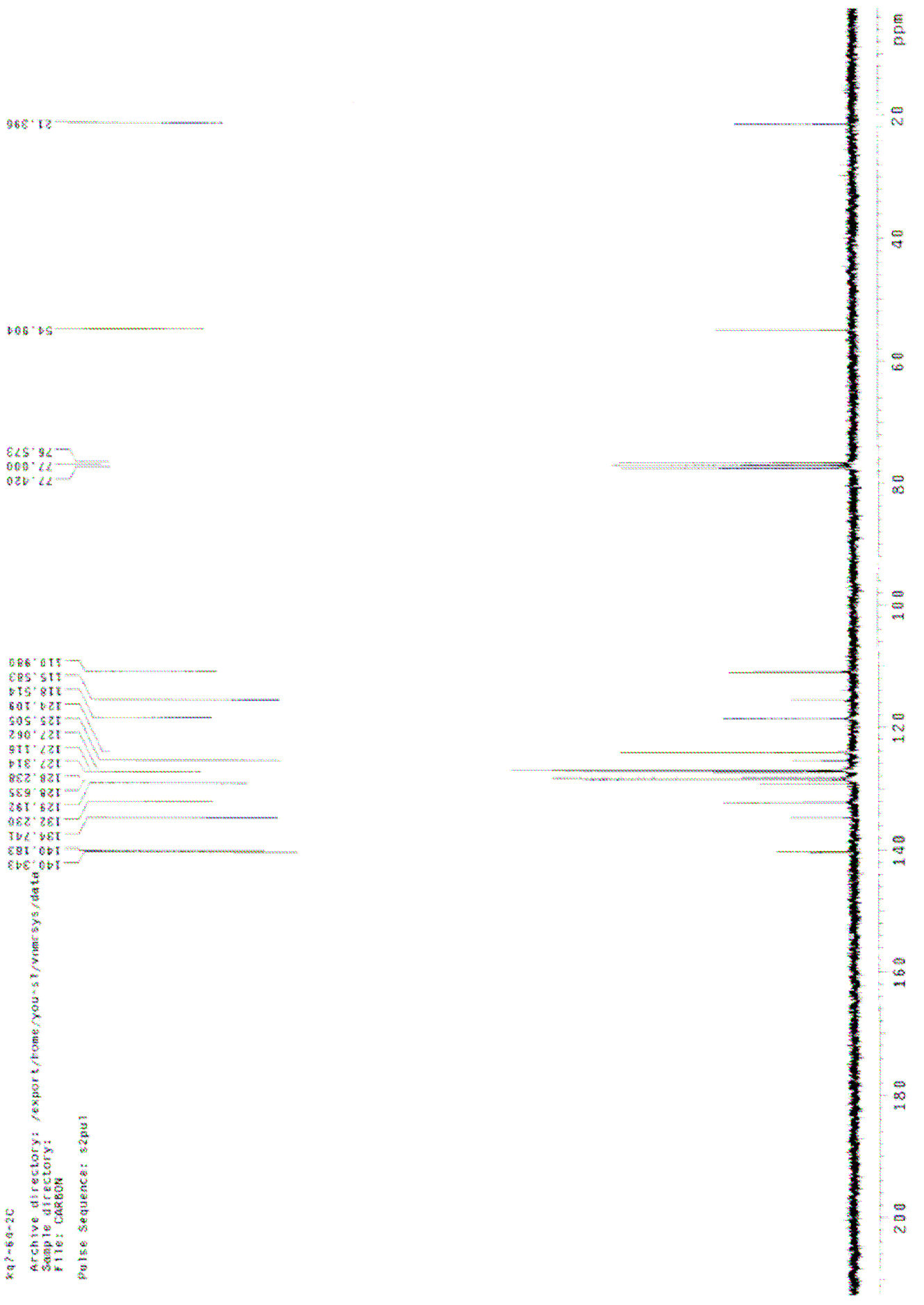
<smiles>FC(F)(F)NC(c1ccccc1)c1c[nH]c2ccc(Br)cc12</smiles>

(entry 7, Table 3): $\mathrm{R}_{\mathrm{f}}=0.30$ (ethyl acetate/petroleum ether $=1 / 2$, v/v); colorless solid, $82 \%$ yield, $98 \%$ ee [Daicel Chiralcel OD-H, Hexanes / IPA $=80 / 20,1.0 \mathrm{ml} \square \mathrm{min}^{-1}, \lambda=254 \mathrm{~nm}, \mathrm{t}$ (major) $=8.81 \mathrm{~min}, \mathrm{t}$ $($ minor $)=25.46 \mathrm{~min}] ;[\alpha]_{\mathrm{D}}{ }^{20}=+23.4^{\mathrm{o}}(\mathrm{c}=1.01$, Acetone $) .{ }^{1} \mathrm{H}$ NMR $(300 \mathrm{MHz}$, DMSO-d 6$) \delta 2.29(\mathrm{~s}, 3 \mathrm{H})$, $5.71(\mathrm{~d}, J=8.4 \mathrm{~Hz}, 1 \mathrm{H}), 6.80(\mathrm{~d}, J=2.4 \mathrm{~Hz}, 1 \mathrm{H}), 7.13-7.30(\mathrm{~m}, 9 \mathrm{H}), 7.36(\mathrm{~d}, J=1.5 \mathrm{~Hz}, 1 \mathrm{H}), 7.51(\mathrm{~d}, J=$ $8.1 \mathrm{~Hz}, 2 \mathrm{H}), 8.51(\mathrm{~d}, J=8.7 \mathrm{~Hz}, 1 \mathrm{H}), 11.09(\mathrm{br}, 1 \mathrm{H}) ;{ }^{13} \mathrm{C}$ NMR $\left(75 \mathrm{MHz}, \mathrm{DMSO}-\mathrm{d}_{6}\right) \delta 20.9,53.9,111.3$, 113.4 115.3, 121.1, 123.6, 125.6, 126.3, 126.7, 126.9, 127.1, 127.9, 128.9, 135.0, 138.5, 141.5, 141.9; MS (EI): m/z (\%relative intensity) 454 (M+, 2), 91 (100), 204 (55), 107 (23). HRMS (MALDI/DHB): Calcd for $\mathrm{C}_{22} \mathrm{H}_{19} \mathrm{BrN}_{2} \mathrm{O}_{2} \mathrm{SNa}^{+}\left(\mathrm{M}+\mathrm{Na}^{+}\right)$: 477.0253. Found: 477.0243. 


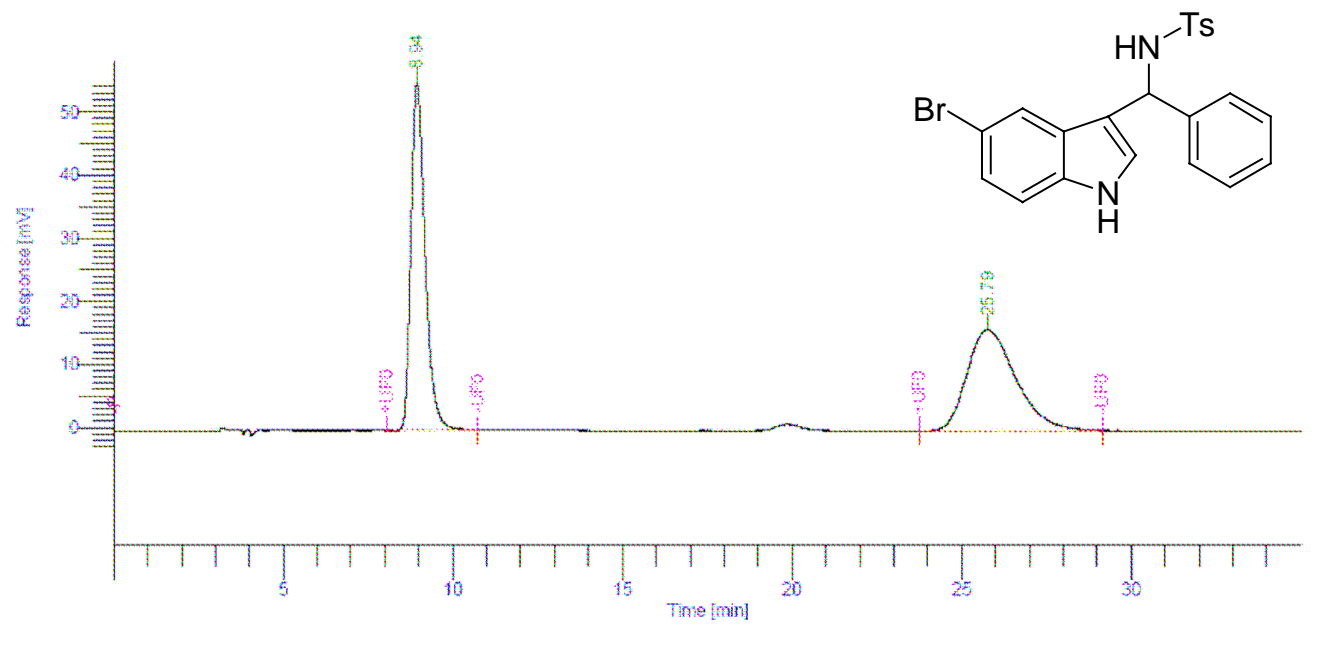

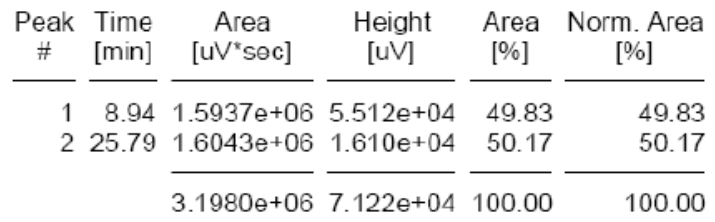

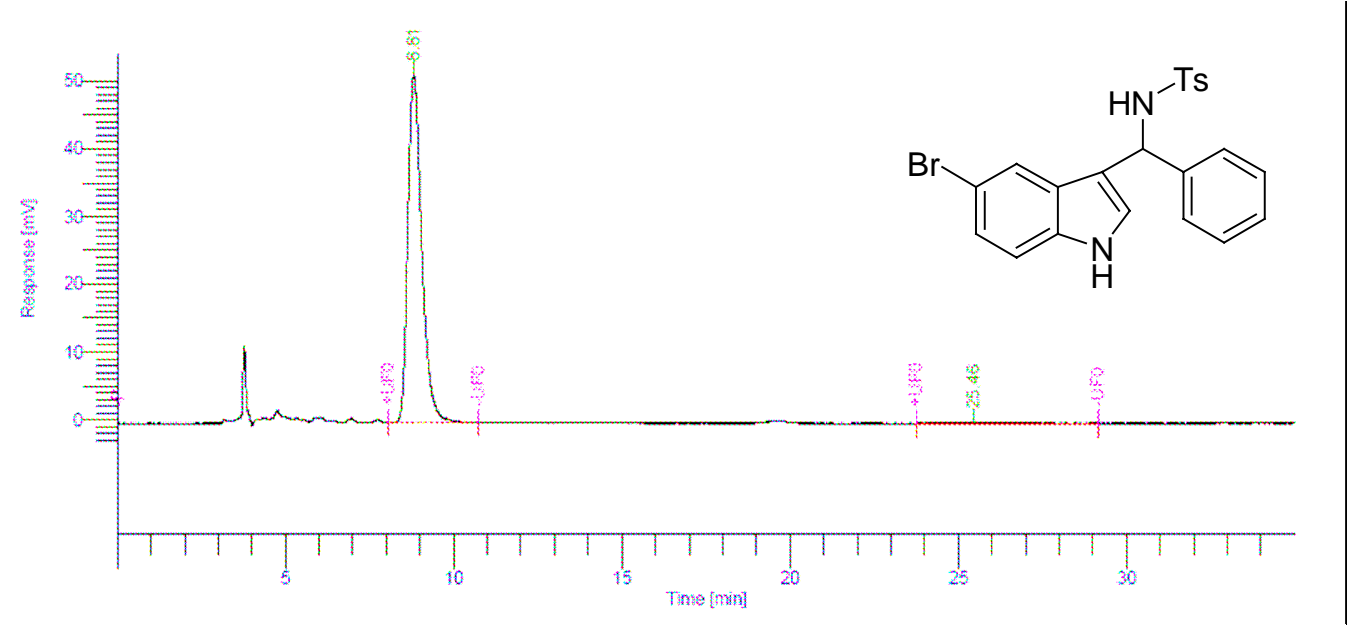

\begin{tabular}{|c|c|c|c|c|c|}
\hline $\begin{array}{c}\text { Peak } \\
\#\end{array}$ & $\begin{array}{l}\text { Time } \\
{[\mathrm{min}]}\end{array}$ & $\begin{array}{c}\text { Area } \\
{\left[\mathrm{uV}^{*} \mathrm{sec}\right]}\end{array}$ & $\begin{array}{l}\text { Height } \\
\text { [uV] }\end{array}$ & $\begin{array}{c}\text { Area } \\
{[\%]}\end{array}$ & $\begin{array}{c}\text { Norm. Area } \\
{[\%]}\end{array}$ \\
\hline \multirow{3}{*}{$\begin{array}{l}1 \\
2\end{array}$} & 8.81 & $1.4759 e+06$ & $5.140 e+04$ & 99.07 & 99.07 \\
\hline & 25.46 & 13793.3294 & 154.6547 & 0.93 & 0.93 \\
\hline & & $1.4897 \mathrm{e}+06$ & $5.155 e+04$ & 100.00 & 100.00 \\
\hline
\end{tabular}

Chiralcel OD-H Column; Hex/i-PrOH: 80/20; Flow rate $=1.0 \mathrm{~mL} / \mathrm{min} ; 254 \mathrm{~nm}$; 
s8z'

ons'

t9ع $\varepsilon$
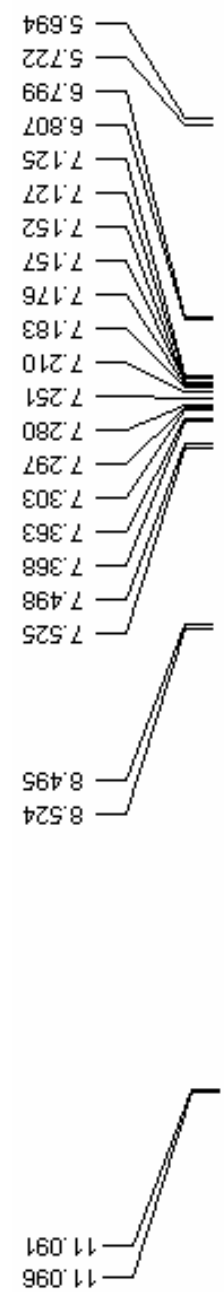

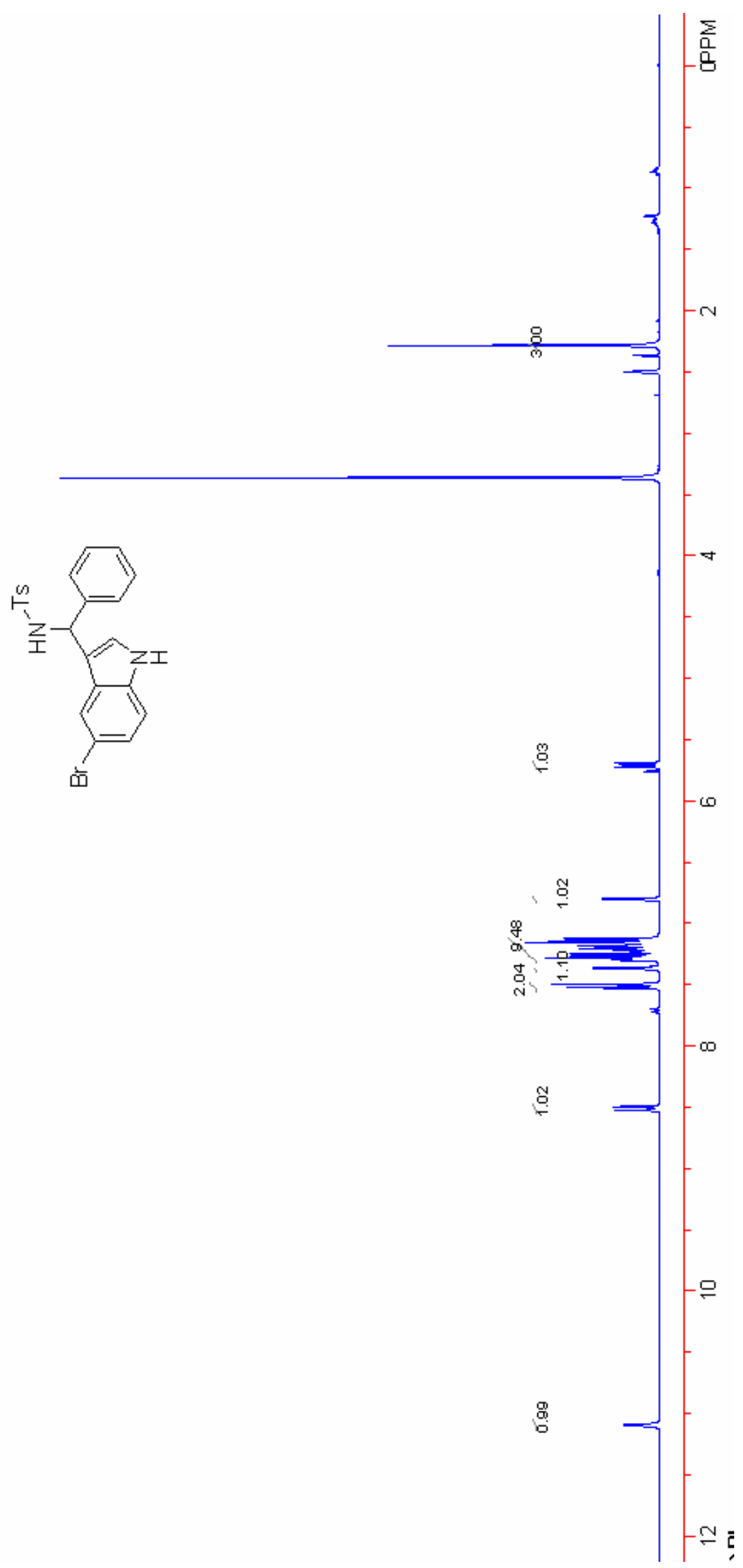


<smiles>FC(F)(F)NC(c1ccccc1)c1c[nH]c2ccc(Br)cc12</smiles>
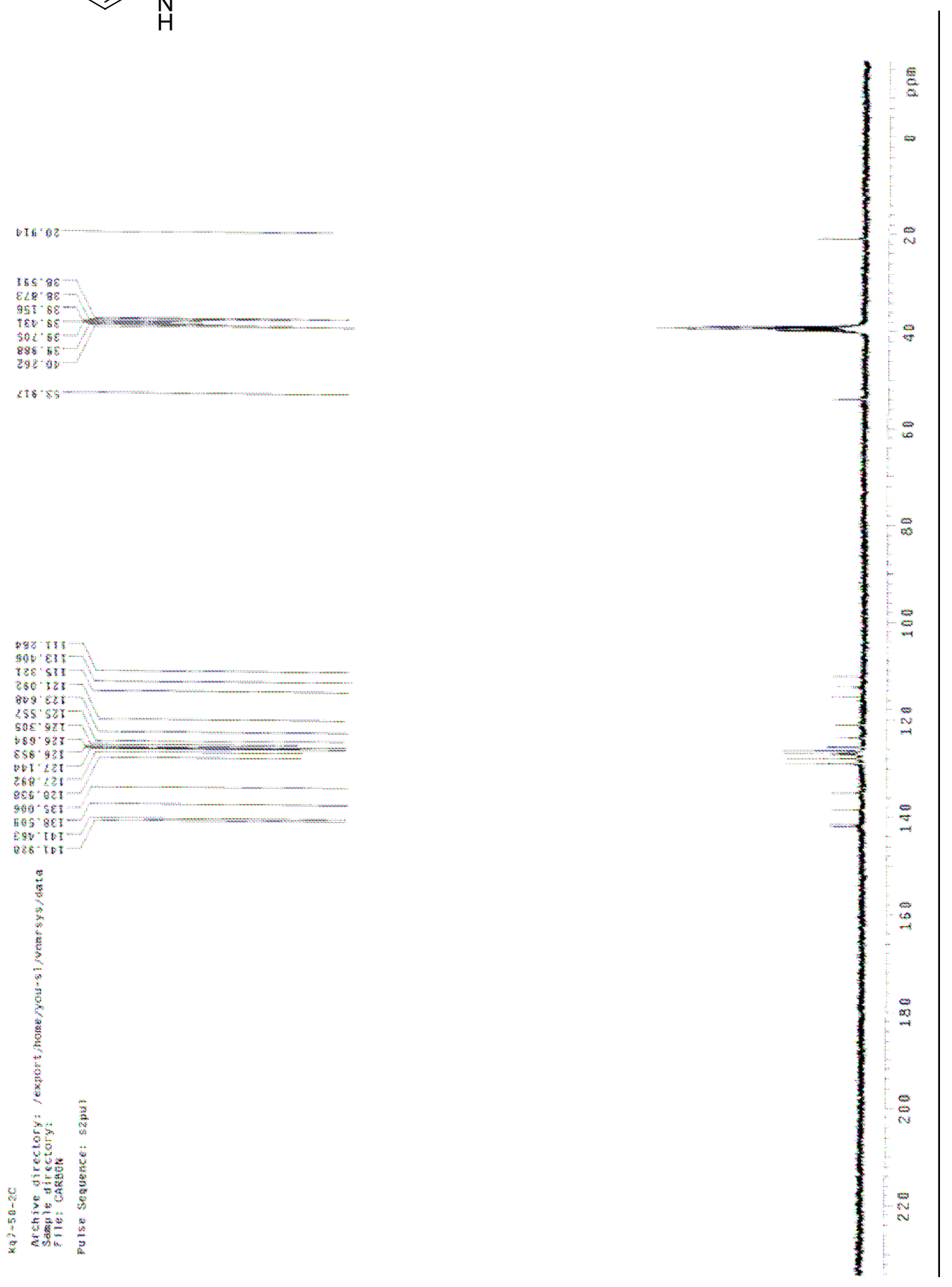
<smiles>[R5]NC(c1ccccc1)c1c[nH]c2ccc(Br)cc12</smiles>

(entry 8, Table 3): $\mathrm{R}_{\mathrm{f}}=0.25$ (ethyl acetate/petroleum ether $=1 / 2$, v/v); colorless solid, $89 \%$ yield, $>99 \%$ ee [Daicel Chiralcel OD-H, Hexanes / IPA $=70 / 30,0.8 \mathrm{ml} \square \mathrm{min}^{-1}, \lambda=254 \mathrm{~nm}, \mathrm{t}$ (major) $=8.03 \mathrm{~min}, \mathrm{t}$ $($ minor $)=19.00 \mathrm{~min}] ;[\alpha]_{\mathrm{D}}{ }^{20}=+27.4^{\mathrm{o}}(\mathrm{c}=1.05$, Acetone $) .{ }^{1} \mathrm{H}$ NMR $\left(300 \mathrm{MHz}, \mathrm{DMSO}-\mathrm{d}_{6}\right) \delta 5.76(\mathrm{~d}, J=$ $9.0 \mathrm{~Hz}, 1 \mathrm{H}), 6.81(\mathrm{~d}, J=2.1 \mathrm{~Hz}, 1 \mathrm{H}), 7.14-7.36(\mathrm{~m}, 9 \mathrm{H}), 7.41-7.49(\mathrm{~m}, 2 \mathrm{H}), 7.62(\mathrm{~d}, J=7.8 \mathrm{~Hz}, 2 \mathrm{H}), 8.63$ $(\mathrm{d}, J=9.0 \mathrm{~Hz}, 1 \mathrm{H}), 11.09$ (br, 1H); ${ }^{13} \mathrm{C}$ NMR $\left(75 \mathrm{MHz}, \mathrm{DMSO}-\mathrm{d}_{6}\right) \delta 54.0,111.3,113.4,115.3,121.1$, 123.6, 125.5, 126.2, 126.7, 126.9, 127.1, 127.9, 128.4, 131.8, 135.0, 141.1, 141.3; MS (EI): m/z (\%relative intensity) 440 ( $\left.\mathrm{M}^{+}, 4\right), 77$ (100), 204 (93), 157 (50), 93 (37). HRMS Calcd for $\left(\mathrm{M}^{+}\right) \mathrm{C}_{21} \mathrm{H}_{17} \mathrm{BrN}_{2} \mathrm{O}_{2} \mathrm{~S}$ : 440.0160. Found: 440.0175. 

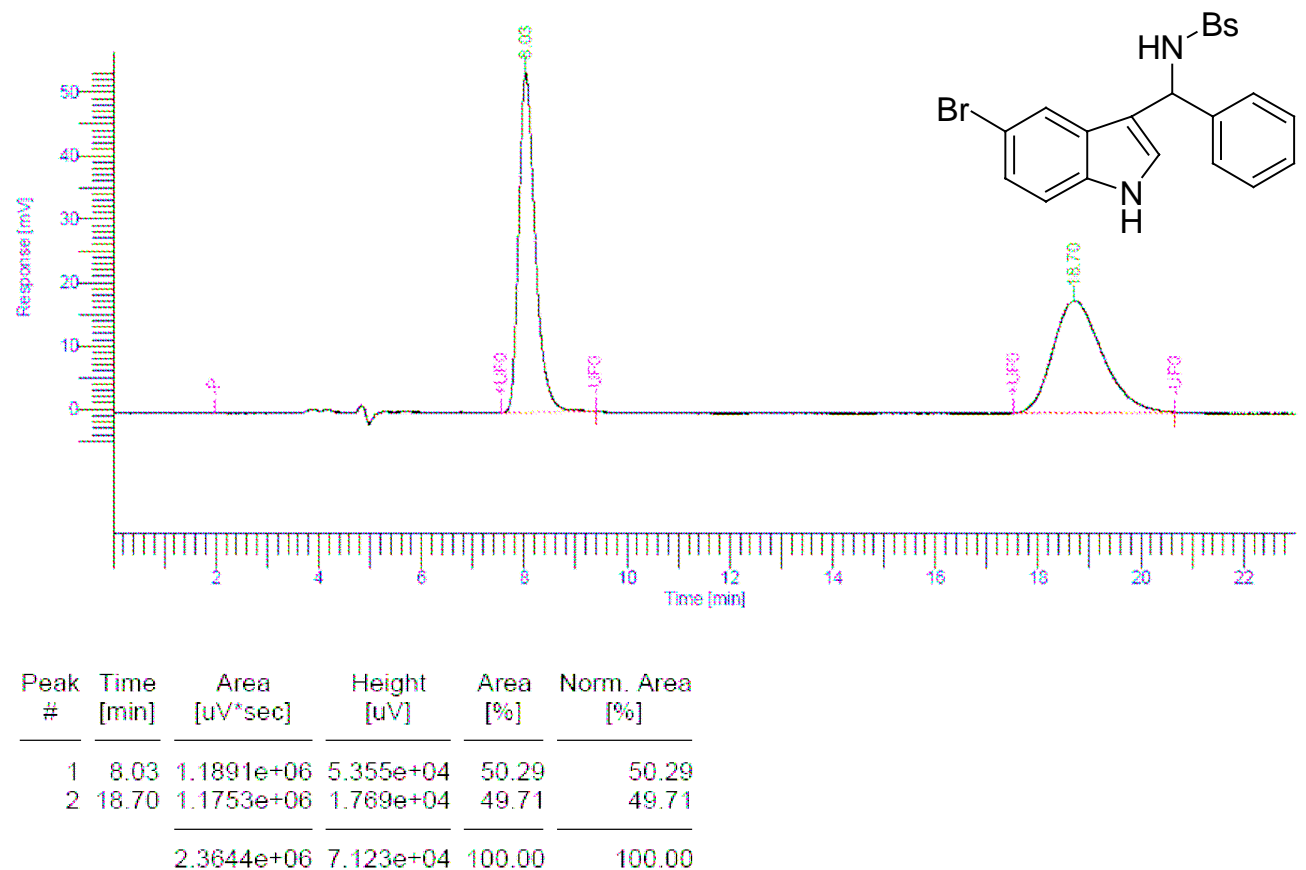

Chiralcel OD-H Column; Hexi-PrOH: 70/30; Flow rate $=0.8 \mathrm{~mL} / \mathrm{min} ; 254 \mathrm{~nm}$;

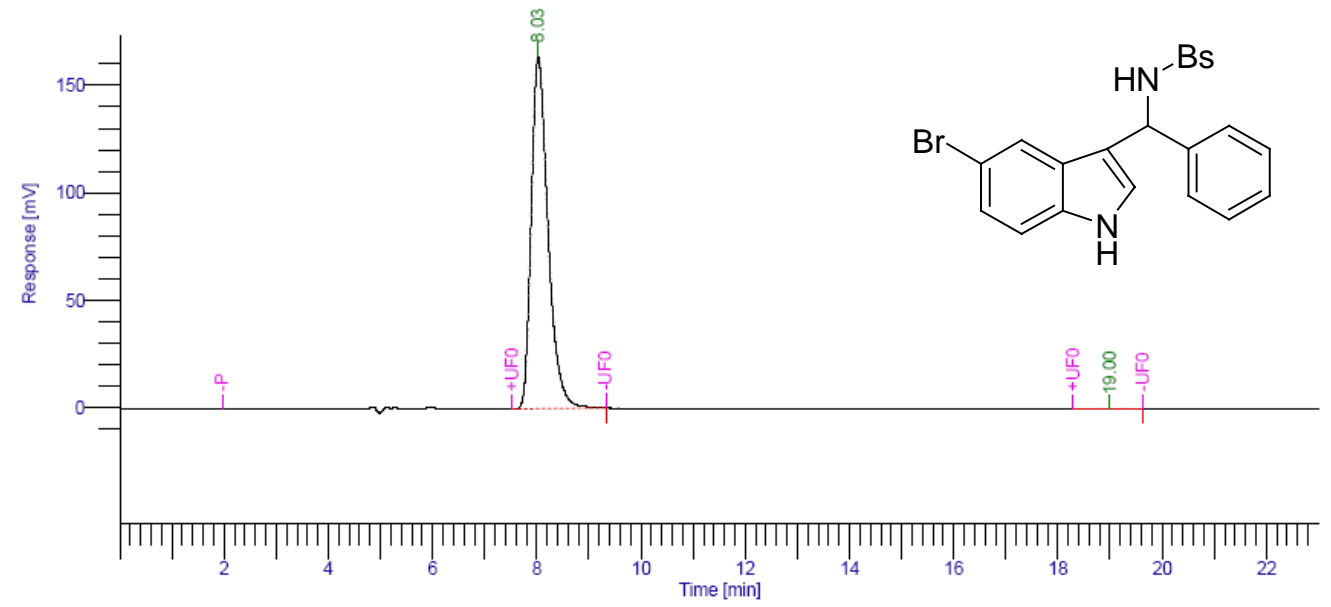

\begin{tabular}{|c|c|c|c|c|c|}
\hline $\begin{array}{c}\text { Peak } \\
\#\end{array}$ & $\begin{array}{l}\text { Time } \\
\text { [min] }\end{array}$ & $\begin{array}{c}\text { Area } \\
{\left[\mathrm{u}^{*} \mathrm{sec}\right]}\end{array}$ & $\begin{array}{l}\text { Height } \\
\text { [uv] }\end{array}$ & $\begin{array}{l}\text { Area } \\
{[\%]}\end{array}$ & $\begin{array}{c}\text { Norm. Area } \\
{[\%]}\end{array}$ \\
\hline 1 & 8.03 & $3.6622 e+06$ & $1.639 e+05$ & 9981 & 9981 \\
\hline \multirow{2}{*}{ 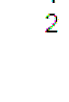 } & 19.00 & 7028.3220 & 183.3896 & 0.19 & 0.19 \\
\hline & & $3.6692 e+06$ & 1.641 & 100.00 & 10 \\
\hline
\end{tabular}

Chiralcel OD-H Column; Hexi-ProH: $70 / 30$; Flow rate $=0.8 \mathrm{~mL} / \mathrm{min} ; 254 \mathrm{~nm}$; 
$00 \mathrm{z}$

$8 s \varepsilon \varepsilon$

tors -

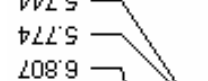

$2089 \longrightarrow$

21897

(2)

$8812-$

$\varepsilon 0 z$

$\angle S Z L-Y$

$9 L Z$

$98 \mathrm{z}$

tos.

$6 Z \varepsilon 2-\%$

SSE

$+1+2 L-1$

$6 e+2-$

Est L -

$\angle 8+2-$

$809 \angle-F$

tc9 $2-$

$\varepsilon 198$

ct9:

เ60เレ

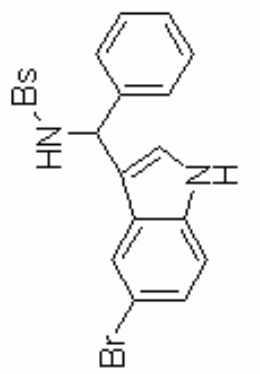

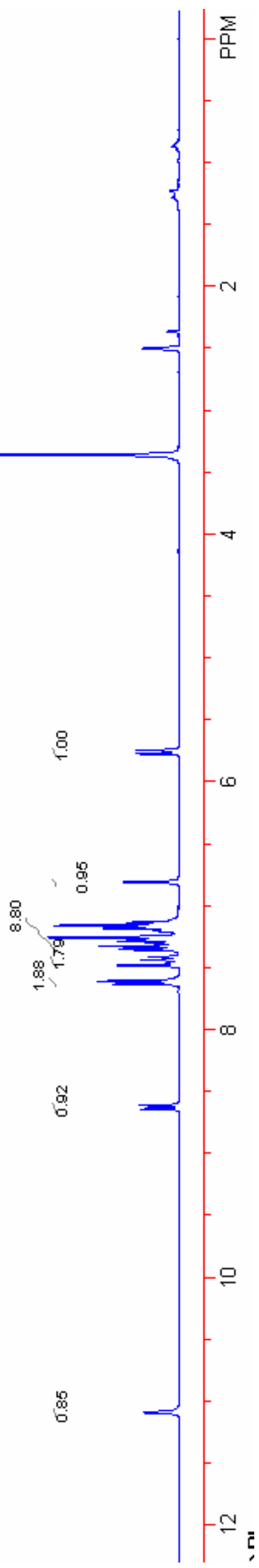


<smiles>S=C(S)NC(c1ccccc1)c1c[nH]c2ccc(Br)cc12</smiles>
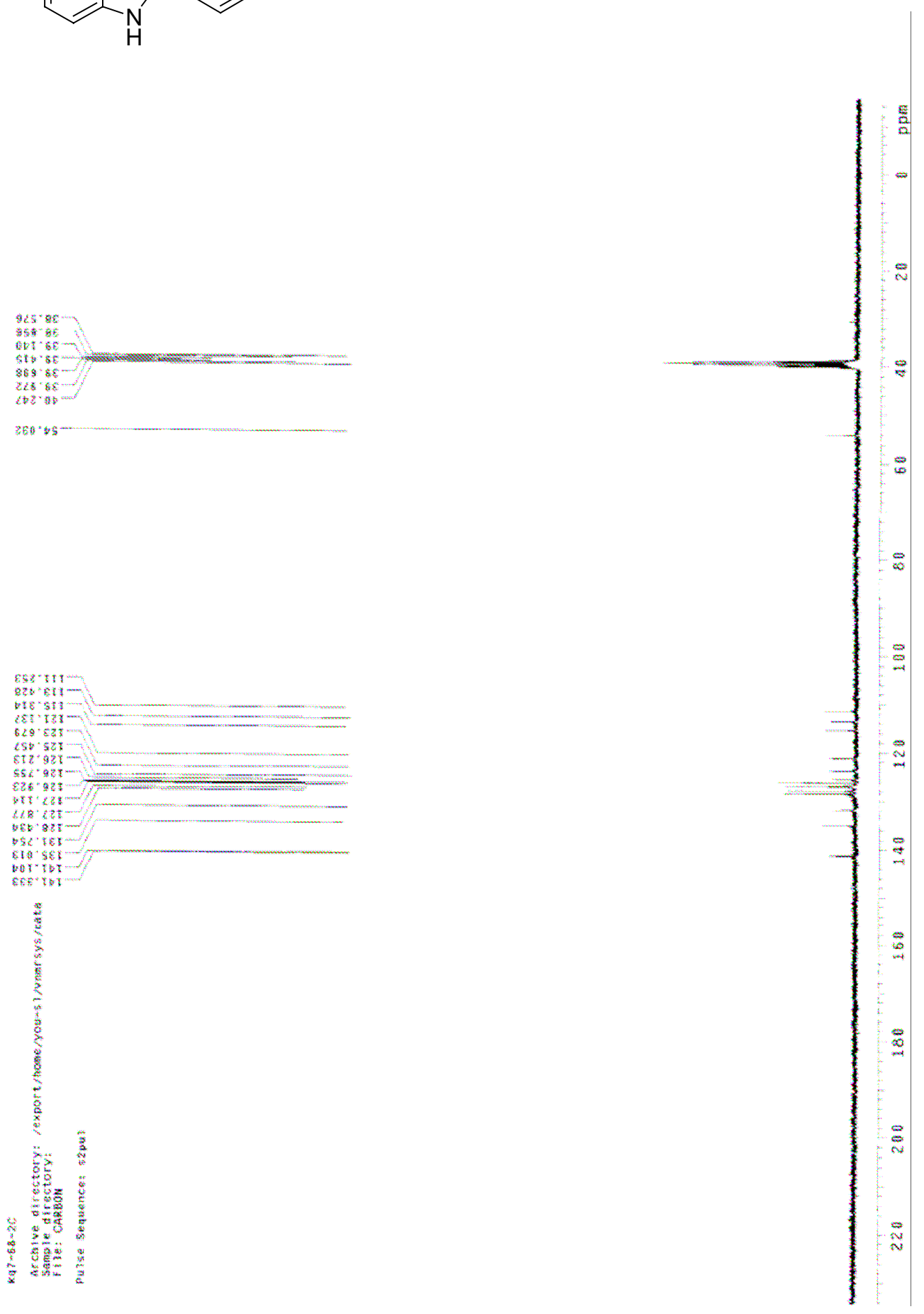


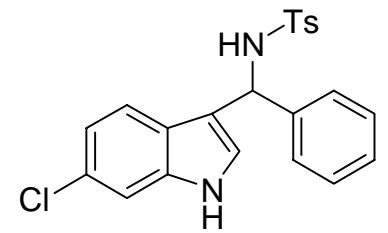

(entry 9, Table 3): $\mathrm{R}_{\mathrm{f}}=0.40$ (ethyl acetate/petroleum ether $=1 / 2, \mathrm{v} / \mathrm{v}$ ); colorless solid, $68 \%$ yield, $98 \%$ ee [Daicel Chiralcel OD-H, Hexanes / IPA $=70 / 30,0.8 \mathrm{ml} \square \mathrm{min}^{-1}, \lambda=254 \mathrm{~nm}, \mathrm{t}$ (major) $=7.25 \mathrm{~min}, \mathrm{t}$ (minor) $=10.19 \mathrm{~min}] ;[\mathrm{\alpha}]_{\mathrm{D}}{ }^{20}=+18.8^{\circ}(\mathrm{c}=1.01$, Acetone $) .{ }^{1} \mathrm{H}$ NMR $\left(300 \mathrm{MHz}, \mathrm{DMSO}-\mathrm{d}_{6}\right) \delta 2.26(\mathrm{~s}, 3 \mathrm{H}), 5.75(\mathrm{~d}$, $J=8.7 \mathrm{~Hz}, 1 \mathrm{H}), 6.85(\mathrm{~d}, J=2.1 \mathrm{~Hz}, 1 \mathrm{H}), 6.92\left(\mathrm{dd}, J_{1}=1.5 \mathrm{~Hz}, J_{2}=8.4 \mathrm{~Hz}, 1 \mathrm{H}\right), 7.09-7.37(\mathrm{~m}, 9 \mathrm{H}), 7.49(\mathrm{~d}$, $J=8.1 \mathrm{~Hz}, 2 \mathrm{H}), 8.54(\mathrm{~d}, J=9.0 \mathrm{~Hz}, 1 \mathrm{H}), 11.03(\mathrm{br}, 1 \mathrm{H}) ;{ }^{13} \mathrm{C}$ NMR $\left(75 \mathrm{MHz}, \mathrm{DMSO}_{-} \mathrm{d}_{6}\right) \delta 20.8,54.2,110.9$, $115.9,118.8,120.2$, 124.1, 124.9, 125.9, 126.2, 126.7, 126.9, 127.9, 128.8, 136.7, 138.6, 141.3, 141.8. 


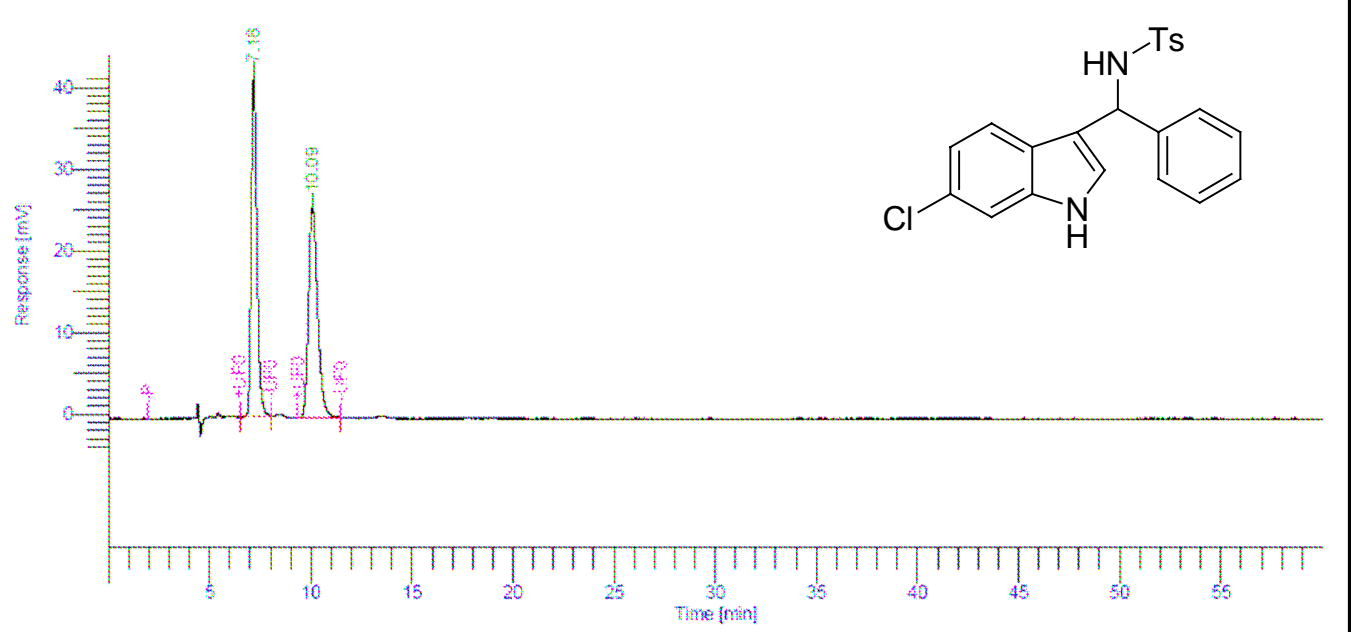

\begin{tabular}{|c|c|c|c|c|c|}
\hline $\begin{array}{c}\text { Peak } \\
\#\end{array}$ & $\begin{array}{l}\text { Time } \\
{[\mathrm{min}]}\end{array}$ & $\begin{array}{c}\text { Area } \\
{\left[\mathrm{uV}^{*} \mathrm{sec}\right]}\end{array}$ & $\begin{array}{l}\text { Height } \\
{[\mathrm{uV}]}\end{array}$ & $\begin{array}{c}\text { Area } \\
{[\%]}\end{array}$ & $\begin{array}{c}\text { Norm. Area } \\
{[\%]}\end{array}$ \\
\hline \multirow{3}{*}{$\begin{array}{l}1 \\
2\end{array}$} & 7.18 & 805842.1615 & $4.159 e+04$ & 49.88 & 49.88 \\
\hline & 10.09 & 809864.7658 & $2.585 e+04$ & 50.12 & 50.12 \\
\hline & & $1.6157 e+06$ & $6.744 e+04$ & 100.00 & 100.00 \\
\hline
\end{tabular}

Chiralcel OD-H Column; Hex/i-PrOH: 70/30; Flow rate $=0.8 \mathrm{~mL} / \mathrm{min} ; 254 \mathrm{~nm}$;

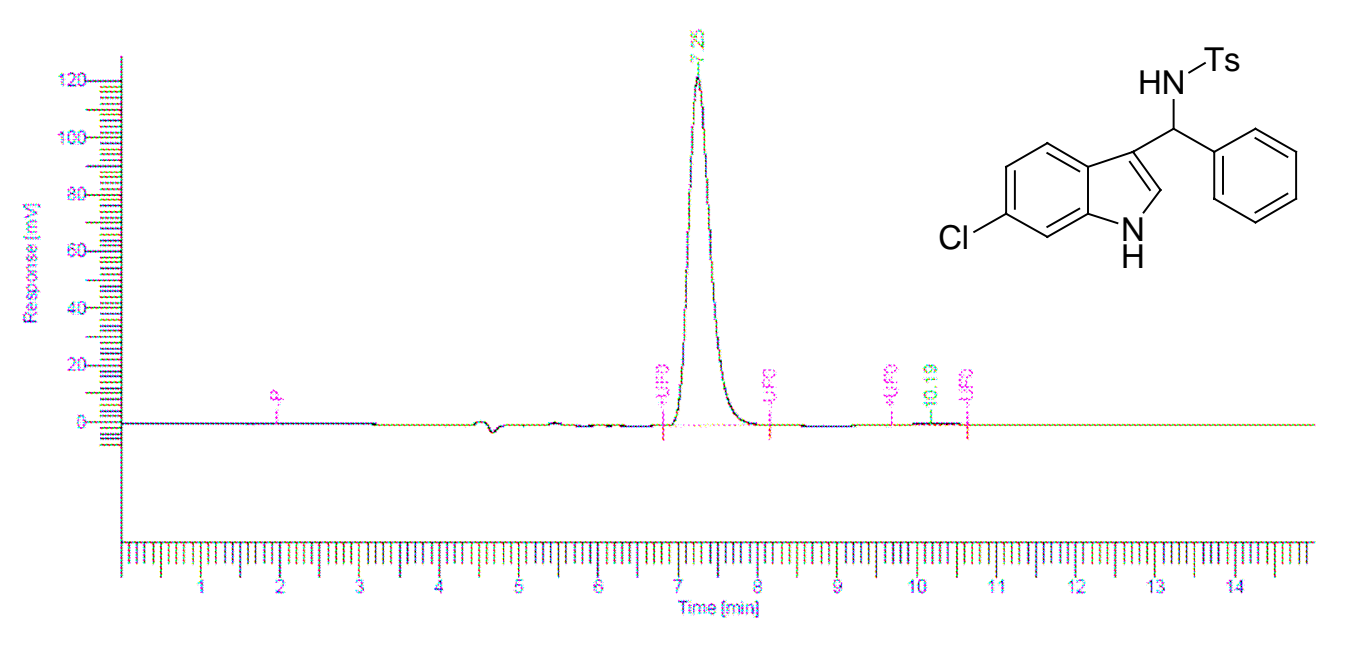

\begin{tabular}{|c|c|c|c|c|c|}
\hline $\begin{array}{c}\text { Peak } \\
\#\end{array}$ & $\begin{array}{l}\text { Time } \\
{[\mathrm{min}]}\end{array}$ & $\begin{array}{c}\text { Area } \\
{\left[\mathrm{uV}^{*} \mathrm{sec}\right]}\end{array}$ & $\begin{array}{l}\text { Height } \\
{[\mathrm{uV}]}\end{array}$ & $\begin{array}{c}\text { Area } \\
{[\%]}\end{array}$ & $\begin{array}{c}\text { Norm. Area } \\
{[\%]}\end{array}$ \\
\hline & 7.25 & $2.3678 e+06$ & $1.225 e+05$ & 99.18 & 99.18 \\
\hline & 10.19 & 19665.5588 & 723.9482 & 0.82 & 0.82 \\
\hline & & $2.3875 e+06$ & $1.232 e^{+05}$ & 100.00 & 100.00 \\
\hline
\end{tabular}

Chiralcel OD-H Column; Hex/i-PrOH: 70/30; Flow rate $=0.8 \mathrm{~mL} / \mathrm{min} ; 254$ nm; 
toz $z$
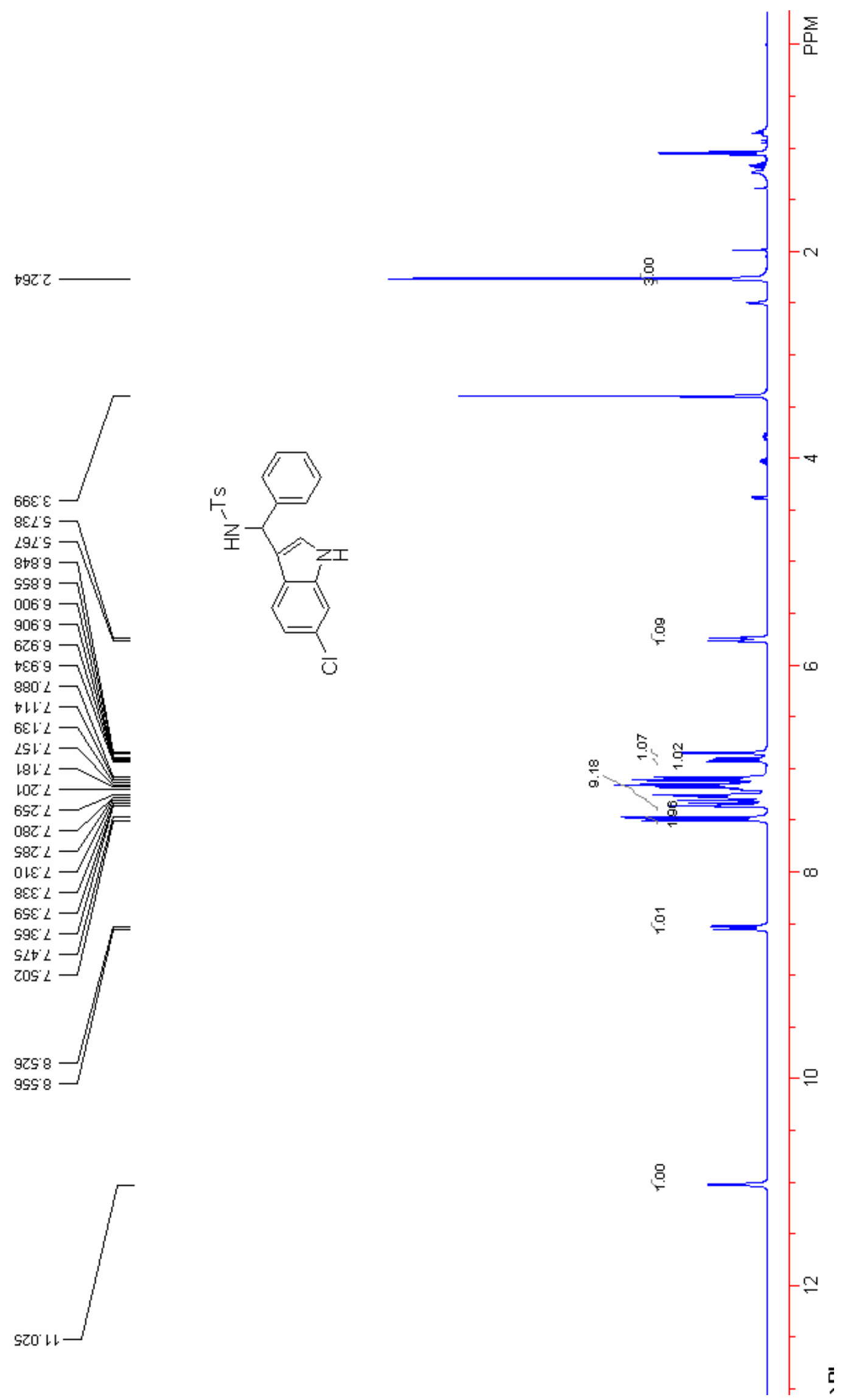

szon 

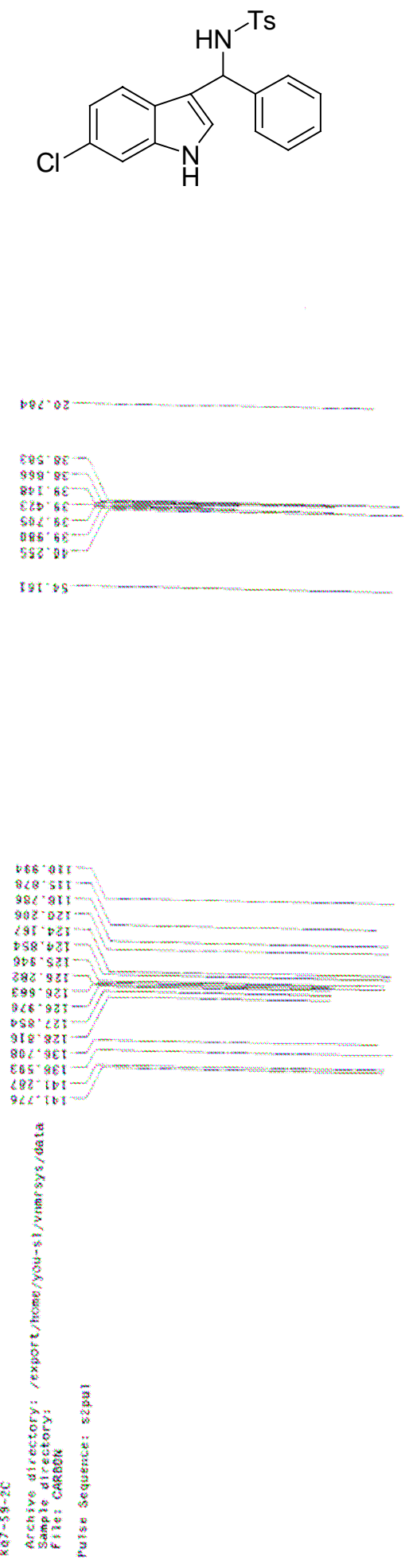

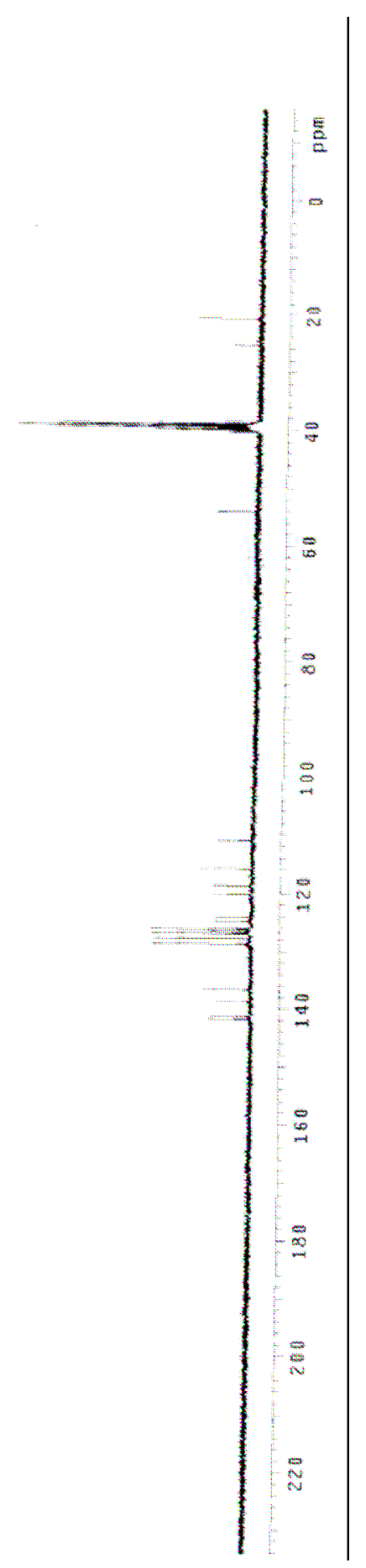




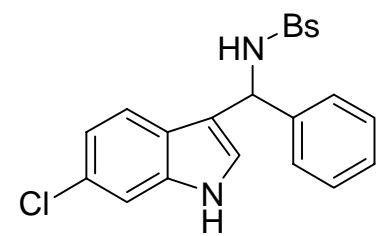

(entry 10, Table 3): $R_{\mathrm{f}}=0.30$ (ethyl acetate/petroleum ether $=1 / 2$, v/v); colorless solid, $87 \%$ yield,

$>99 \%$ ee [Daicel Chiralcel OD-H, Hexanes $/ \mathrm{IPA}=70 / 30,0.8 \mathrm{ml} \square \mathrm{min}^{-1}, \lambda=254 \mathrm{~nm}, \mathrm{t}$ (major) $=11.59$ $\min , \mathrm{t}($ minor $)=17.13 \mathrm{~min}] ;[\alpha]_{\mathrm{D}}{ }^{20}=+18.4^{\mathrm{o}}\left(\mathrm{c}=0.99\right.$, Acetone). ${ }^{1} \mathrm{H}$ NMR $\left(300 \mathrm{MHz}, \mathrm{DMSO}-\mathrm{d}_{6}\right) \delta 5.80(\mathrm{~d}$, $J=9.0 \mathrm{~Hz}, 1 \mathrm{H}), 6.84(\mathrm{~d}, J=2.4 \mathrm{~Hz}, 1 \mathrm{H}), 6.95\left(\mathrm{dd}, J_{1}=1.8 \mathrm{~Hz}, J_{2}=8.4 \mathrm{~Hz}, 1 \mathrm{H}\right), 7.13-7.19(\mathrm{~m}, 3 \mathrm{H})$, 7.26-7.43 (m, 7H), $7.64(\mathrm{~d}, J=7.5 \mathrm{~Hz}, 2 \mathrm{H}), 8.67$ (d, $J=9.0 \mathrm{~Hz}, 1 \mathrm{H}), 11.04(\mathrm{br}, 1 \mathrm{H}) ;{ }^{13} \mathrm{C} \mathrm{NMR}(75 \mathrm{MHz}$, DMSO-d $\left._{6}\right) \delta 54.2,111.1,115.9,118.9,120.2,124.2,124.9,126.0,126.2,126.8,126.9,127.9,128.4,131.7$, 136.7, 141.1, 141.4; MS (EI): m/z (\%relative intensity) 396 (M+, 4), 204 (100), 239 (81), 77 (56). HRMS Calcd for $\left(\mathrm{M}^{+}\right) \mathrm{C}_{21} \mathrm{H}_{17} \mathrm{ClN}_{2} \mathrm{O}_{2} \mathrm{~S}: 396.0699$. Found: 396.0710. 


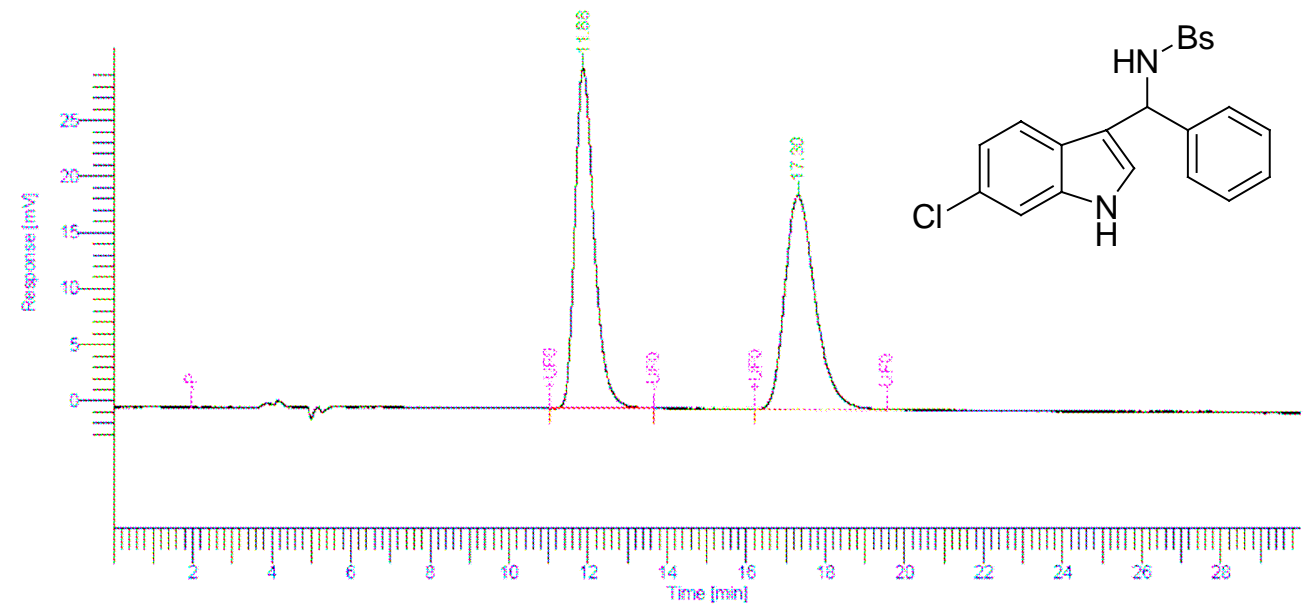

\begin{tabular}{|c|c|c|c|c|c|}
\hline $\begin{array}{c}\text { Peak } \\
\text { \# }\end{array}$ & $\begin{array}{l}\text { Time } \\
\text { [min] }\end{array}$ & $\begin{array}{c}\text { Area } \\
{\left[u^{*} v^{*} \text { sec] }\right.}\end{array}$ & $\begin{array}{l}\text { Height } \\
\text { [uV] }\end{array}$ & $\begin{array}{c}\text { Area } \\
{[\%]}\end{array}$ & $\begin{array}{c}\text { Nom Area } \\
{[\%]}\end{array}$ \\
\hline 1 & 11 & 101 & 3.02 & 99 & \\
\hline 2 & $\$ 7.30$ & $1.0183 e+06$ & $1.915 \mathrm{e}+04$ & 50.01 & 50 . \\
\hline & & 0361 & $4.944 \mathrm{e}+04$ & 100.00 & 10 \\
\hline
\end{tabular}

Chiralcel OD-H Column; Hexi-ProH: $7030 ;$ Flow rate $=0.8 \mathrm{~mL} / \mathrm{min} ; 254 \mathrm{~nm}$

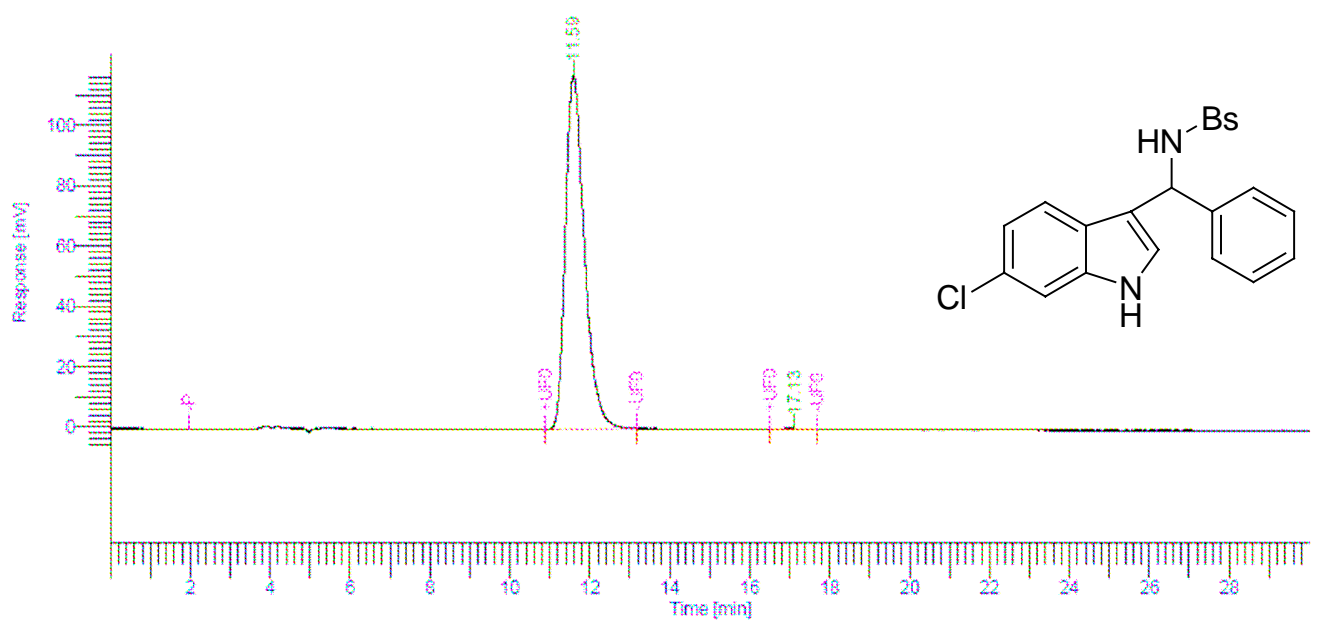

\begin{tabular}{|c|c|c|c|c|c|}
\hline $\begin{array}{c}\text { Peak } \\
\#\end{array}$ & $\begin{array}{l}\text { Time } \\
{[\mathrm{min}]}\end{array}$ & $\begin{array}{c}\text { Area } \\
{\left[\mathrm{u}^{*} \sec \right]}\end{array}$ & $\begin{array}{l}\text { Height } \\
{[u V]}\end{array}$ & $\begin{array}{c}\text { Area } \\
{[\%]}\end{array}$ & $\begin{array}{c}\text { Nom. Area } \\
{[\%]}\end{array}$ \\
\hline 1 & 11.59 & $3.88210+06$ & $1.174 \mathrm{e}+05$ & 99.73 & 99.73 \\
\hline 2 & 17.13 & 10364.2609 & 270.8093 & 0.27 & 0.27 \\
\hline
\end{tabular}

Chiralcel OD-H Column; Hexi-PrOH: $70 / 30$; Flow rate $=0.8 \mathrm{~mL} / \mathrm{min} ; 254 \mathrm{~nm}$ 


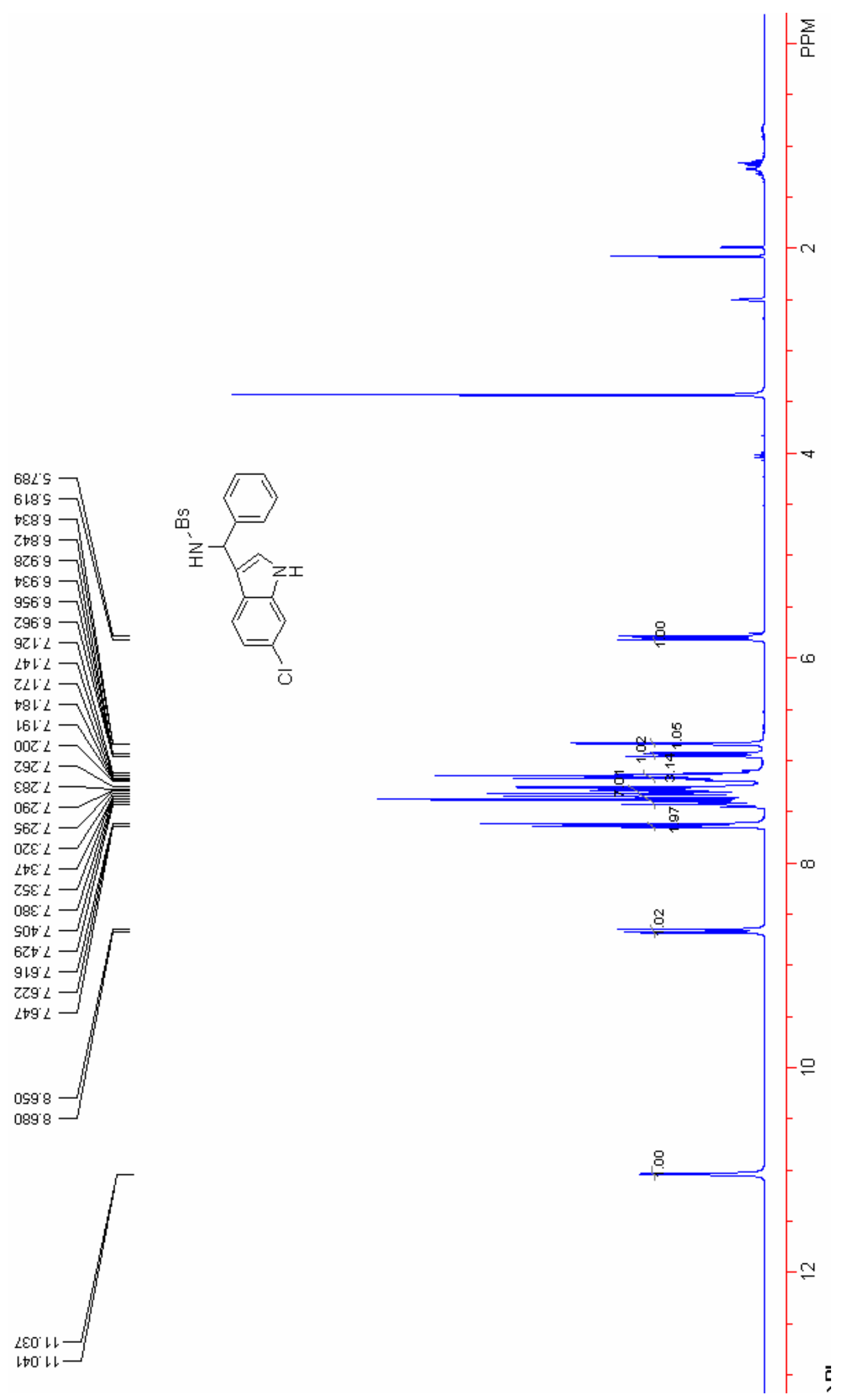



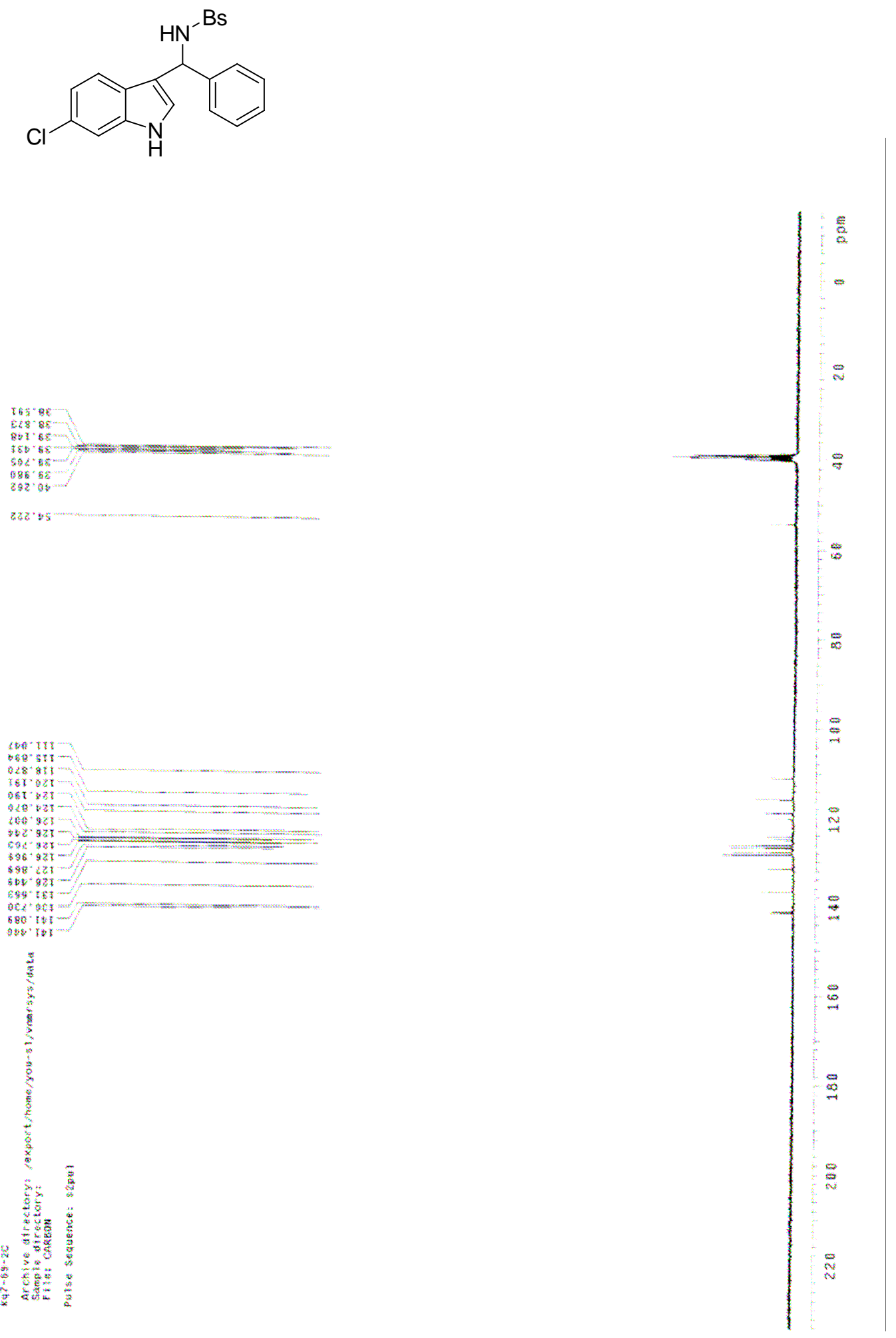


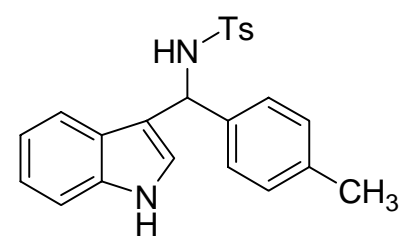

(entry 11, Table 3): $\mathrm{R}_{\mathrm{f}}=0.50$ (ethyl acetate/petroleum ether $=1 / 2, \mathrm{v} / \mathrm{v}$ ); colorless solid, $93 \%$ yield, $>99 \%$ ee [Daicel Chiralcel OD-H, Hexanes $/ \mathrm{IPA}=70 / 30,0.8 \mathrm{ml} \square \mathrm{min}^{-1}, \lambda=254 \mathrm{~nm}, \mathrm{t}$ (major) $=9.33 \mathrm{~min}, \mathrm{t}$ $($ minor $)=16.71 \mathrm{~min}] ;[\alpha]_{\mathrm{D}}{ }^{20}=+12.1^{\mathrm{o}}(\mathrm{c}=1.0$, Acetone $) .{ }^{1} \mathrm{H}$ NMR $\left(300 \mathrm{MHz}, \mathrm{CDCl}_{3}\right) \delta 2.26(\mathrm{~s}, 3 \mathrm{H}), 2.33$ $(\mathrm{s}, 3 \mathrm{H}), 5.27(\mathrm{~m}, 1 \mathrm{H}), 5.76(\mathrm{~d}, J=7.2 \mathrm{~Hz}, 1 \mathrm{H}), 6.61(\mathrm{~m}, 1 \mathrm{H}), 6.92-6.96(\mathrm{~m}, 3 \mathrm{H}), 7.02-7.10(\mathrm{~m}, 4 \mathrm{H}), 7.12(\mathrm{~d}$, $J=7.2 \mathrm{~Hz}, 1 \mathrm{H}), 7.22(\mathrm{~d}, J=8.1 \mathrm{~Hz}, 2 \mathrm{H}), 7.50\left(\mathrm{dd}, J_{1}=1.8 \mathrm{~Hz}, J_{2}=8.1 \mathrm{~Hz}, 2 \mathrm{H}\right), 8.03(\mathrm{br}, 1 \mathrm{H}) ;{ }^{13} \mathrm{C} \mathrm{NMR}$ $\left(75 \mathrm{MHz}, \mathrm{CDCl}_{3}\right) \delta 20.9,21.4,54.8,111.3,116.2,119.2,119.7,122.2,123.8,125.3,127.1,128.9,129.1$, 136.5, 136.9, 137.2, 137.3, 142.9; MS (EI): m/z (\%relative intensity) 390 (M+, 3), 91 (100), 171 (58), 107 (24). HRMS Calcd for $\left(\mathrm{M}^{+}\right) \mathrm{C}_{23} \mathrm{H}_{22} \mathrm{~N}_{2} \mathrm{O}_{2} \mathrm{~S}: 390.1402$. Found: 390.1398. 


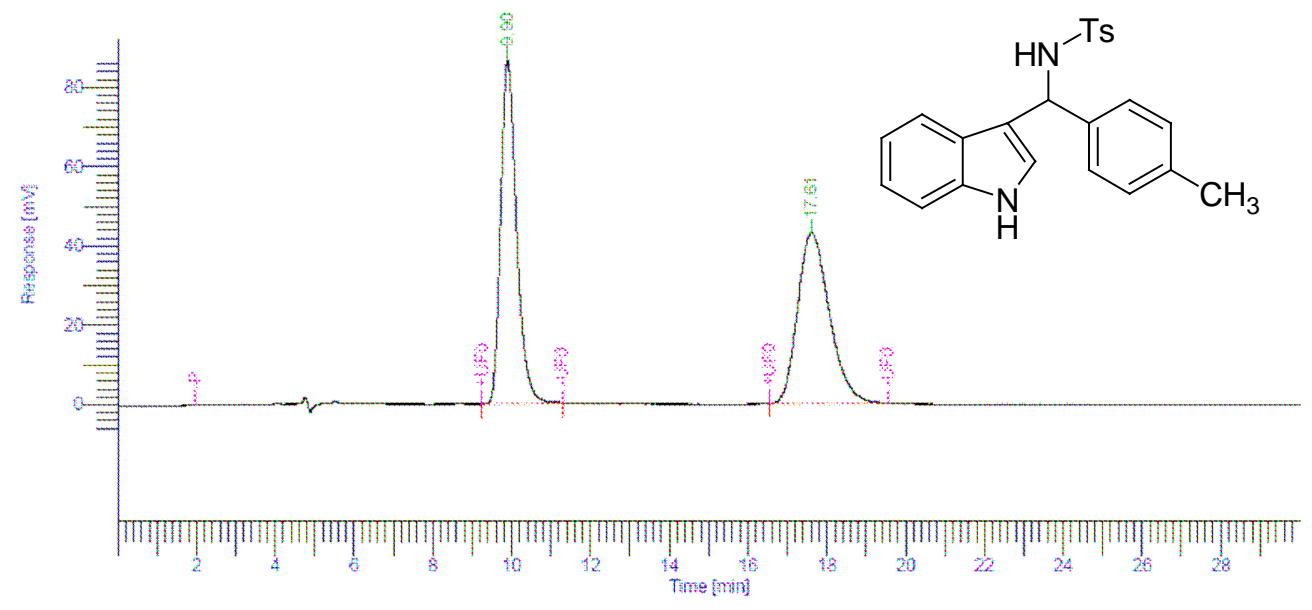

\begin{tabular}{|c|c|c|c|c|c|}
\hline $\begin{array}{c}\text { Peak } \\
=\end{array}$ & $\begin{array}{l}\text { Time } \\
\text { [min] }\end{array}$ & $\begin{array}{c}\text { Area } \\
{\left[u V^{\prime \prime s e c]}\right.}\end{array}$ & $\begin{array}{l}\text { Height } \\
\text { [uV] }\end{array}$ & $\begin{array}{c}\text { Area } \\
{[\%]}\end{array}$ & $\begin{array}{c}\text { Norm Area } \\
{[\%]}\end{array}$ \\
\hline \multirow{3}{*}{2} & 9.90 & $2.4326 e+06$ & $8.673 e+04$ & 50.16 & 50.16 \\
\hline & 17.61 & $2.4166 e+06$ & $4.311 e+04$ & 49.84 & 49.84 \\
\hline & & $4.8492 e+06$ & $1.298 e+05$ & 100.00 & 100.00 \\
\hline
\end{tabular}

Chiralcel OD-H Column; Hex/i-PrOH: $70 / 30 ;$ Flow rate $=0.8 \mathrm{~mL} / \mathrm{min} ; 254 \mathrm{~nm}$;

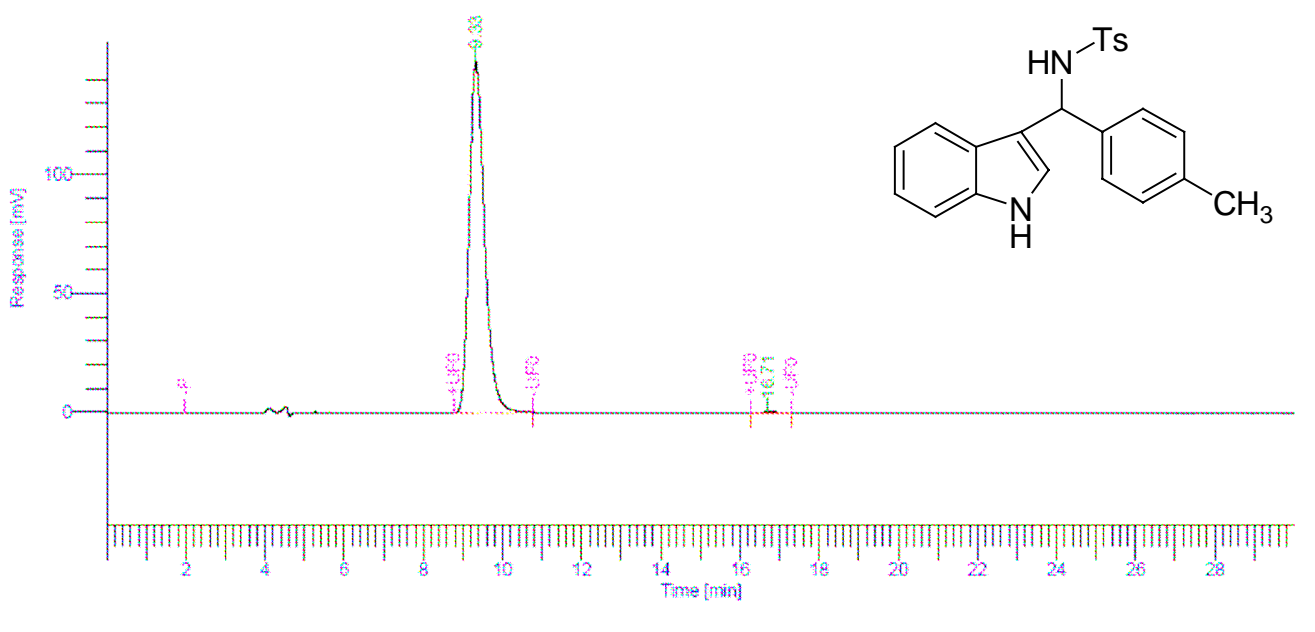

\begin{tabular}{|c|c|c|c|c|c|}
\hline $\begin{array}{l}\text { Peak } \\
\#\end{array}$ & $\begin{array}{l}\text { Time } \\
{[\mathrm{min}]}\end{array}$ & $\begin{array}{c}\text { Area } \\
{\left[\mathrm{uV}^{*} \mathrm{sec}\right]}\end{array}$ & $\begin{array}{l}\text { Height } \\
{[\mathrm{uV}]}\end{array}$ & $\begin{array}{l}\text { Area } \\
{[\%]}\end{array}$ & $\begin{array}{c}\text { Norm. Area } \\
{[\%]}\end{array}$ \\
\hline \multirow{3}{*}{$\begin{array}{l}1 \\
2\end{array}$} & 9.33 & $3.9880 \mathrm{e}+06$ & $1.476 e+05$ & 99.71 & 99.71 \\
\hline & 16.71 & 11491.1619 & 335.4699 & 0.29 & 0.29 \\
\hline & & 3.9995 & 1.47 & 00.00 & \\
\hline
\end{tabular}

Chiralcel OD-H Column; Hex/i-PrOH: 70/30; Flow rate $=0.8 \mathrm{~mL} / \mathrm{min} ; 254 \mathrm{~nm}$; 


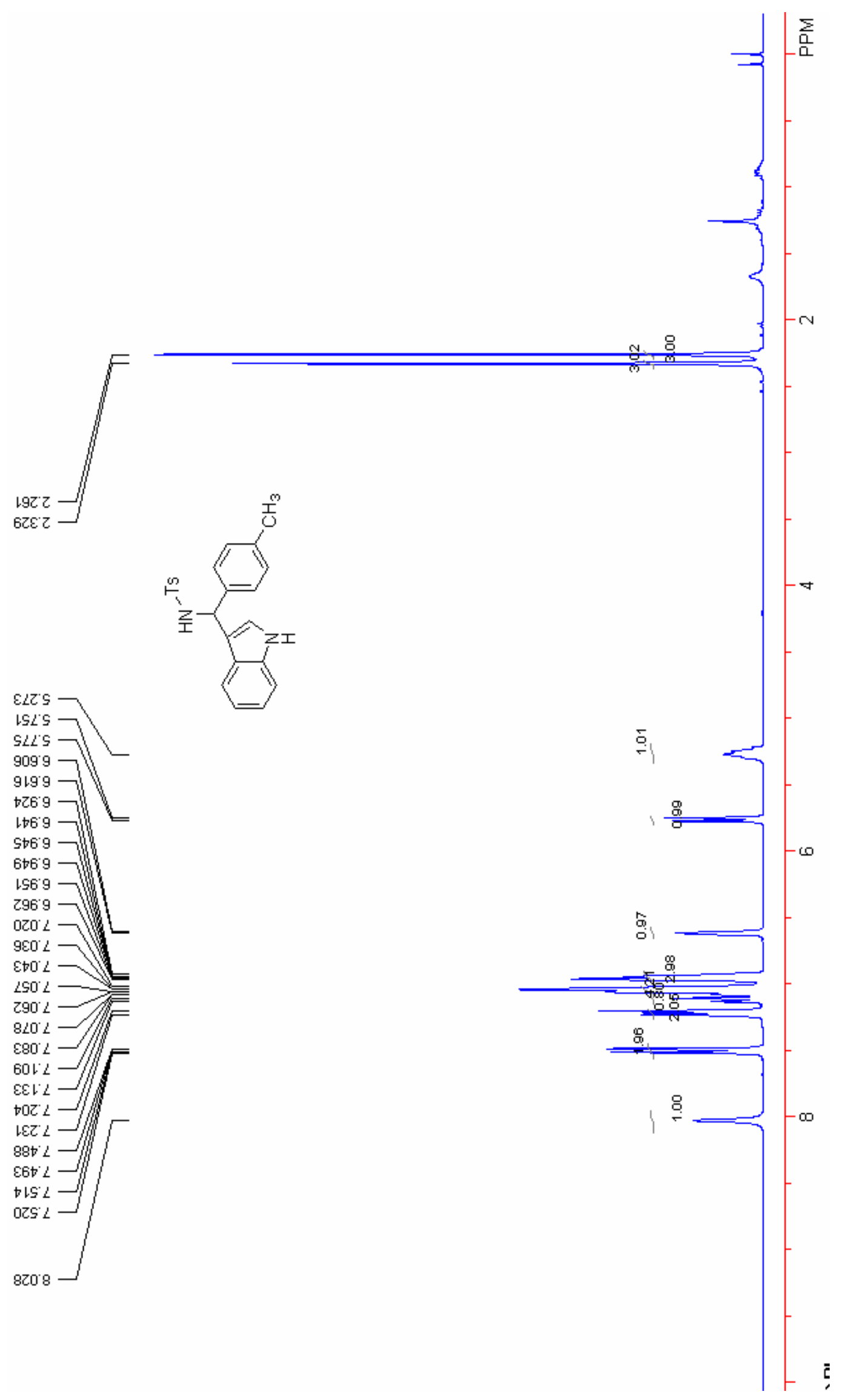



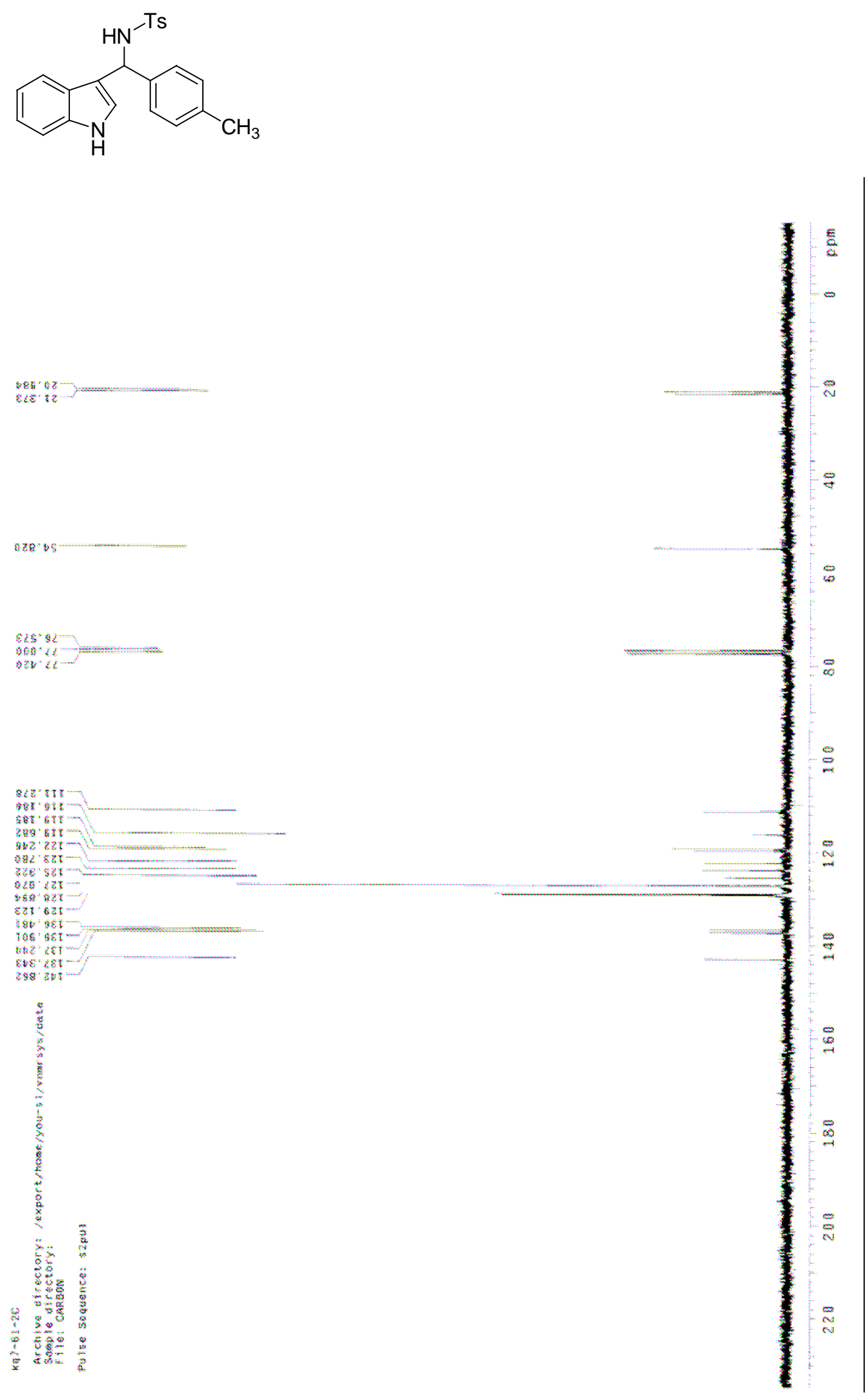


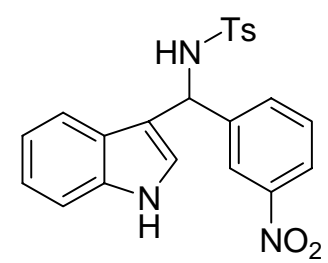

(entry 12, Table 3): $\mathrm{R}_{\mathrm{f}}=0.20$ (ethyl acetate/petroleum ether $=1 / 2, \mathrm{v} / \mathrm{v}$ ); pale yellow solid, $85 \%$ yield, $89 \%$ ee [Daicel Chiralcel OD-H, Hexanes / IPA $=70 / 30,0.8 \mathrm{ml} \square \mathrm{min}^{-1}, \lambda=254 \mathrm{~nm}, \mathrm{t}$ (major) $=26.24 \mathrm{~min}, \mathrm{t}$ $($ minor $)=33.48 \mathrm{~min}] ;[\alpha]_{\mathrm{D}}{ }^{20}=-4.2^{\circ}(\mathrm{c}=0.6$, Acetone $) .{ }^{1} \mathrm{H}$ NMR $\left(300 \mathrm{MHz}, \mathrm{DMSO}-\mathrm{d}_{6}\right) \delta 2.24(\mathrm{~s}, 3 \mathrm{H}), 5.92$ $(\mathrm{d}, J=9.0 \mathrm{~Hz}, 1 \mathrm{H}), 6.77(\mathrm{~d}, J=2.7 \mathrm{~Hz}, 1 \mathrm{H}), 6.94(\mathrm{t}, J=7.2 \mathrm{~Hz}, 1 \mathrm{H}), 7.05-7.10(\mathrm{~m}, 3 \mathrm{H}), 7.31-7.49(\mathrm{~m}, 5 \mathrm{H})$, 7.71-7.74 (m, 1H), $7.99\left(\mathrm{dd}, J_{1}=2.4 \mathrm{~Hz}, J_{2}=8.4 \mathrm{~Hz}, 1 \mathrm{H}\right), 8.07(\mathrm{~s}, 1 \mathrm{H}), 8.68(\mathrm{~d}, J=9.0 \mathrm{~Hz}, 1 \mathrm{H}), 10.99$ (br, $1 \mathrm{H}) ;{ }^{13} \mathrm{C}$ NMR $\left(75 \mathrm{MHz}, \mathrm{DMSO}-\mathrm{d}_{6}\right) \delta 20.7,53.7,111.5,114.6,118.7,118.8,121.4,121.5,121.7,124.0$, $125.2,126.4,128.9,129.3,133.9,136.4,138.3,142.0,143.4,147.4$. 


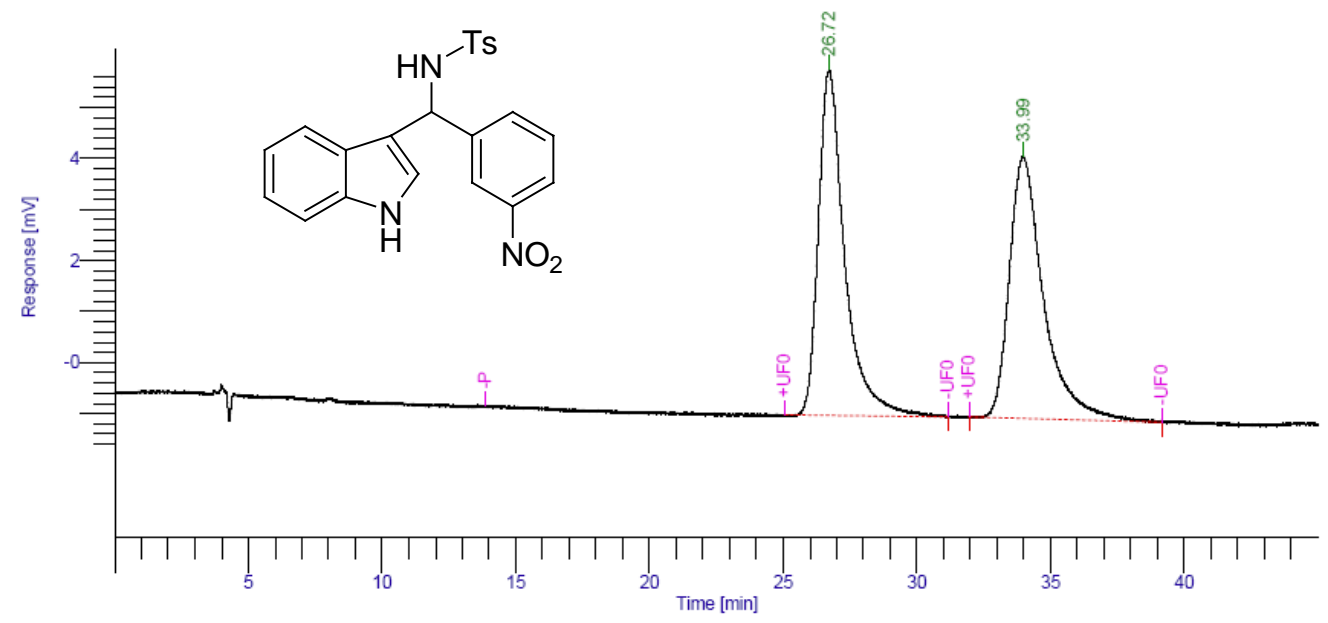

\begin{tabular}{|c|c|c|c|c|c|}
\hline $\begin{array}{c}\text { Peak } \\
\text { \# }\end{array}$ & $\begin{array}{l}\text { Time } \\
\text { [minl] }\end{array}$ & $\begin{array}{c}\text { Area } \\
{\left[\mathrm{uV}^{* *} \mathrm{sec}\right]}\end{array}$ & $\begin{array}{l}\text { Height } \\
\text { [uV] }\end{array}$ & $\begin{array}{c}\text { Area } \\
{[\%]}\end{array}$ & $\begin{array}{c}\text { Nom Area } \\
{[\%]}\end{array}$ \\
\hline \multirow{3}{*}{2} & 26.72 & 463768.5248 & 6776.9968 & 50.33 & 50.33 \\
\hline & 33.99 & 457602.4684 & 5141.6975 & 49.67 & 49.67 \\
\hline & & 9213709932 & $1.192 e+04$ & 100.00 & 100.00 \\
\hline
\end{tabular}

Chiralcel AD-H Column; Hexi-PrOH: $70 / 30 ;$ Flow rate $=0.8 \mathrm{~mL} / \mathrm{min} ; 254 \mathrm{~nm}$;

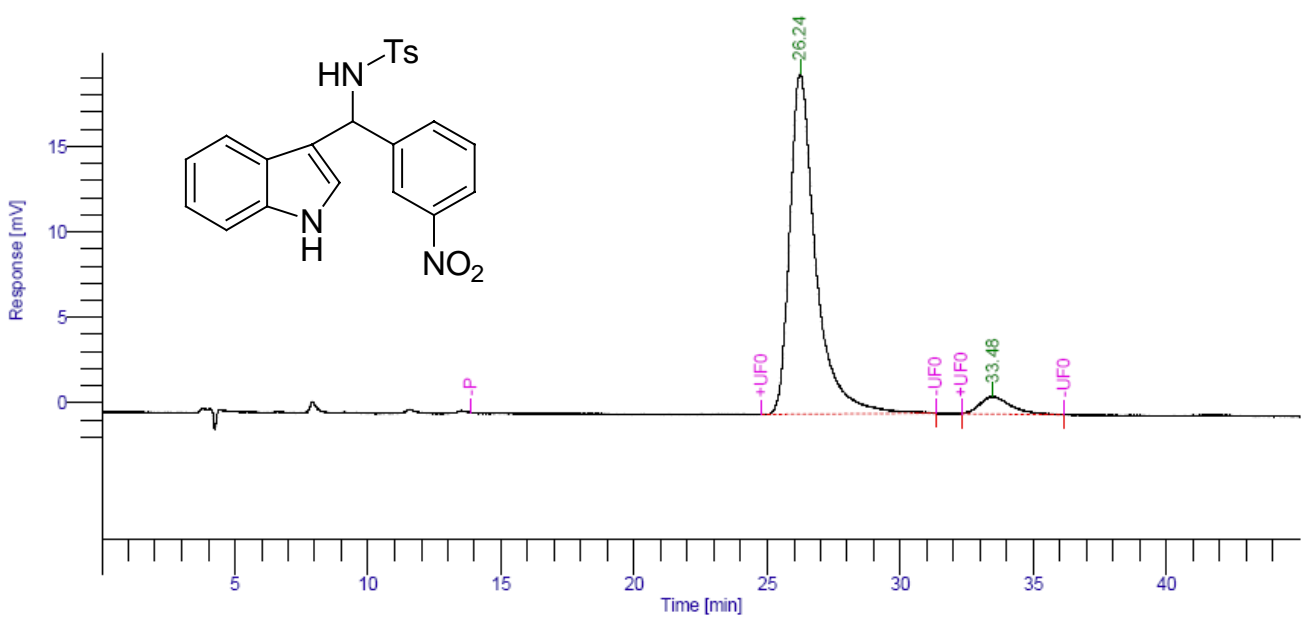

$$
\begin{aligned}
& \text { Peak Time Area Height Area Norm. Area } \\
& \stackrel{\stackrel{\#}{*}}{1} \frac{[\mathrm{min}]}{26.24} \frac{\left[\mathrm{uV}^{*} \mathrm{sec}\right]}{1.3505 \mathrm{e}+06} \frac{[\mathrm{uV}]}{1.988 \mathrm{e}+04} \frac{[\%]}{94.30} \frac{[\%]}{94.30}
\end{aligned}
$$

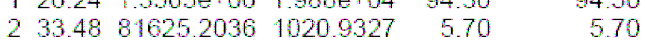

$$
\begin{aligned}
& \overline { 1 . 4 3 2 1 e + 0 6 } \overline { 2 . 0 9 0 e + 0 4 } \overline { 1 0 0 . 0 0 } \longdiv { 1 0 0 . 0 0 }
\end{aligned}
$$

Chralcel AD-H Column; Hexil-PrOH: $70 / 30 ;$ Flow rate $=0.8 \mathrm{~mL} / \mathrm{min} ; 254 \mathrm{~nm}$; 


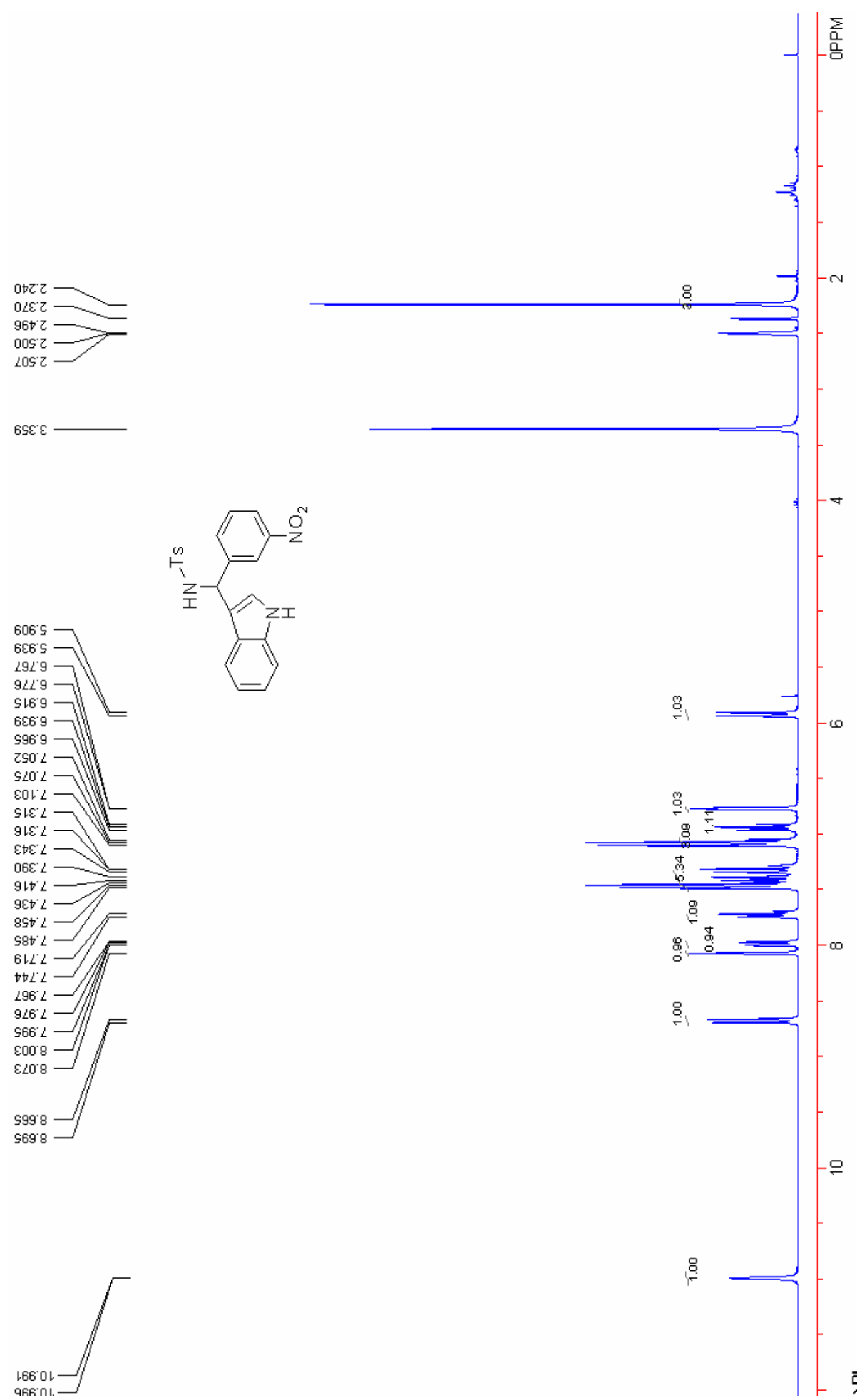



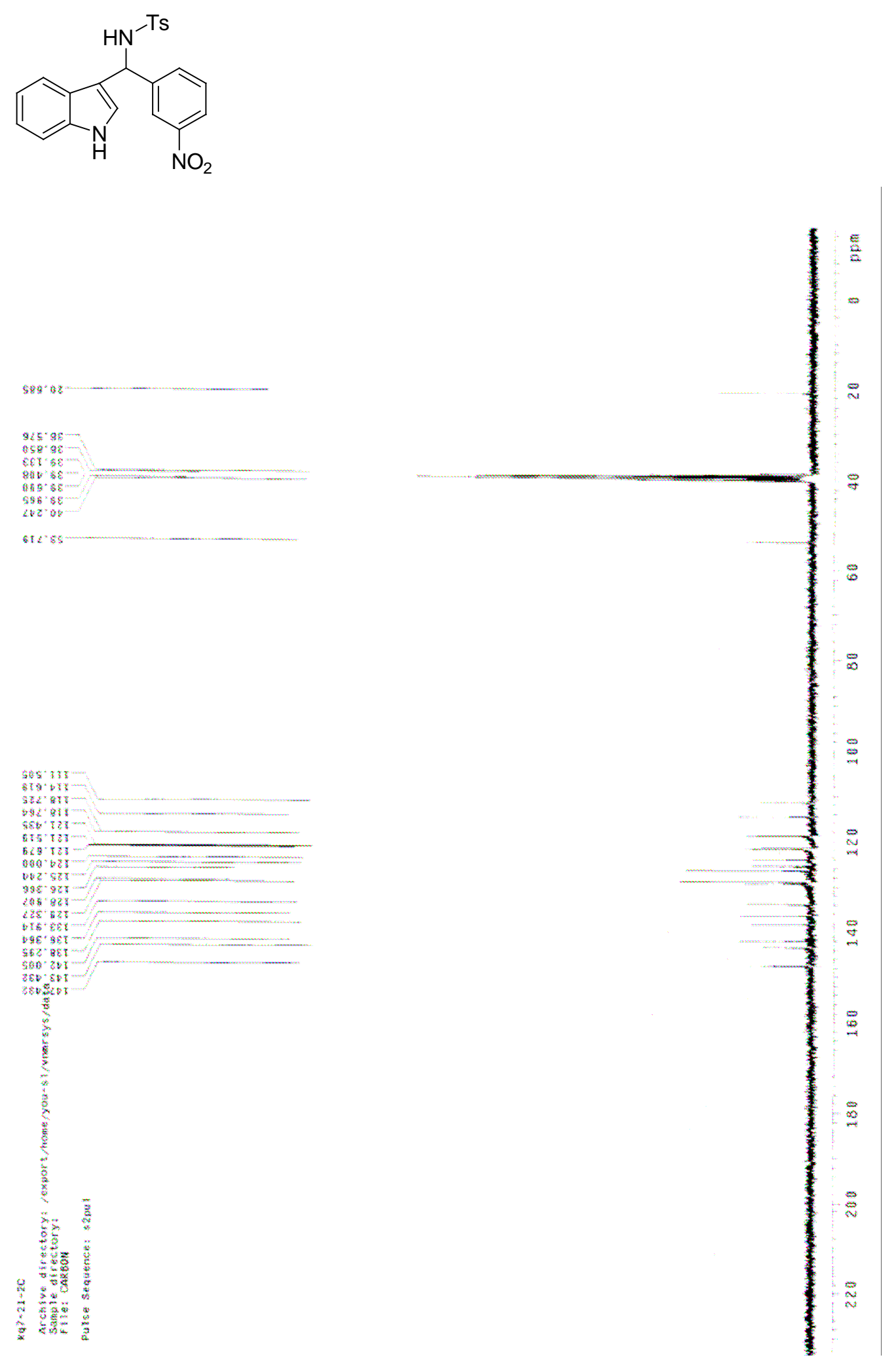


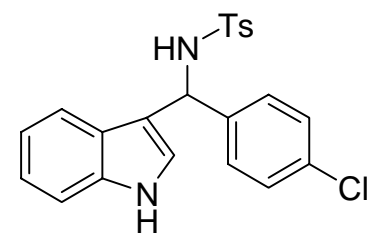

(entry 13 , Table 3 ): $R_{\mathrm{f}}=0.40$ (ethyl acetate/petroleum ether $=1 / 2, \mathrm{v} / \mathrm{v}$ ); colorless solid, $91 \%$ yield, 94\% ee [Daicel Chiralcel OD-H, Hexanes $/ \mathrm{IPA}=80 / 20,1.0 \mathrm{ml} \square \mathrm{min}^{-1}, \lambda=254 \mathrm{~nm}, \mathrm{t}$ (major) $=15.13 \mathrm{~min}$, $\mathrm{t}($ minor $)=28.47 \mathrm{~min}] ;[\alpha]_{\mathrm{D}}{ }^{20}=+16.7^{\circ}(\mathrm{c}=1.3$, Acetone $) .{ }^{1} \mathrm{H}$ NMR $\left(300 \mathrm{MHz}, \mathrm{CDCl}_{3}\right) \delta 2.39(\mathrm{~s}, 3 \mathrm{H}), 5.07$ $(\mathrm{d}, J=5.7 \mathrm{~Hz}, 1 \mathrm{H}), 5.82(\mathrm{~d}, J=6.6 \mathrm{~Hz}, 1 \mathrm{H}), 6.64(\mathrm{~s}, 1 \mathrm{H}), 7.01(\mathrm{t}, J=7.2 \mathrm{~Hz}, 1 \mathrm{H}), 7.12-7.21(\mathrm{~m}, 8 \mathrm{H}), 7.31$ $(\mathrm{d}, J=8.4 \mathrm{~Hz}, 1 \mathrm{H}), 7.56(\mathrm{~d}, J=8.1 \mathrm{~Hz}, 2 \mathrm{H}), 8.02(\mathrm{br}, 1 \mathrm{H})$. 


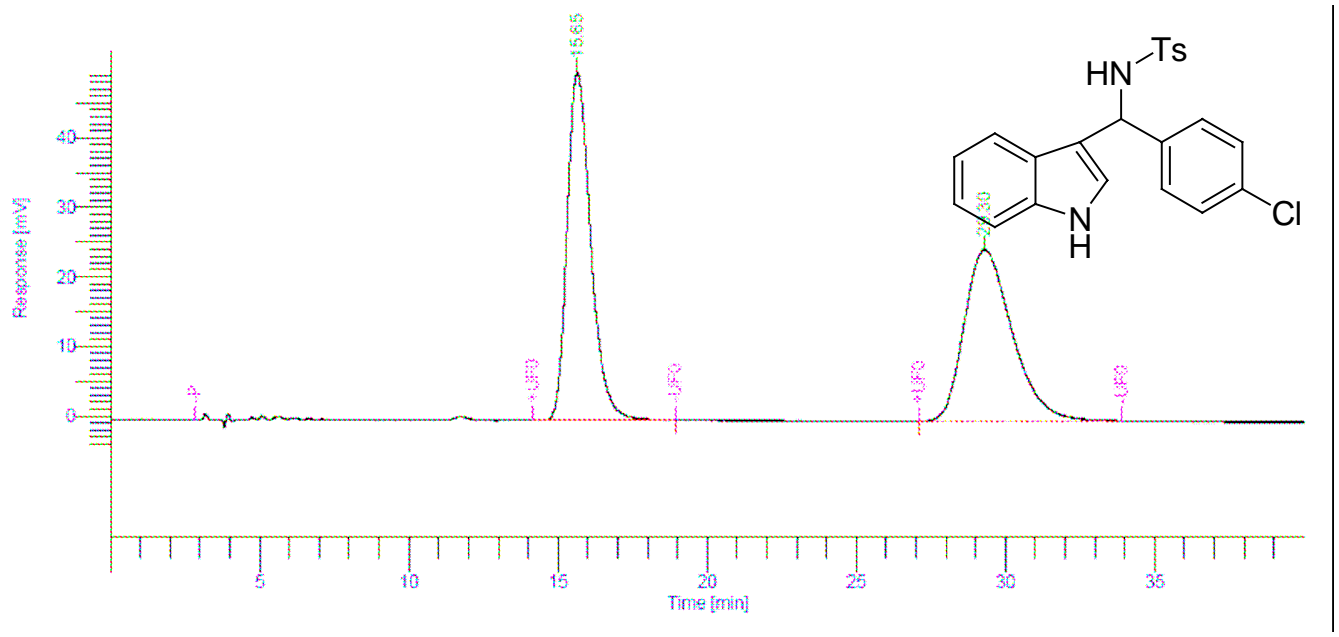

\begin{tabular}{|c|c|c|c|c|c|}
\hline $\begin{array}{c}\text { Peak } \\
\#\end{array}$ & $\begin{array}{l}\text { Time } \\
\text { [min] }\end{array}$ & $\begin{array}{c}\text { Area } \\
{\left[\mathrm{uV}^{*} \mathrm{sec}\right]}\end{array}$ & $\begin{array}{c}\text { Height } \\
{[\mathrm{uV}]}\end{array}$ & $\begin{array}{c}\text { Area } \\
{[\%]}\end{array}$ & $\begin{array}{c}\text { Norm. Area } \\
{[\%]}\end{array}$ \\
\hline \multirow{3}{*}{2} & 15.65 & $2.7517 e+06$ & $5.007 e+04$ & 49.94 & 49.94 \\
\hline & 29.30 & $2.7579 e+06$ & $2.464 e+04$ & 50.06 & 50.06 \\
\hline & & $5.5096 e+06$ & $7.471 \mathrm{e}+04$ & 100.00 & 100.0 \\
\hline
\end{tabular}

Chiralcel OD-H Column; Hex/i-PrOH: 80/20; Flow rate $=1.0 \mathrm{~mL} / \mathrm{min} ; 254$ nm;

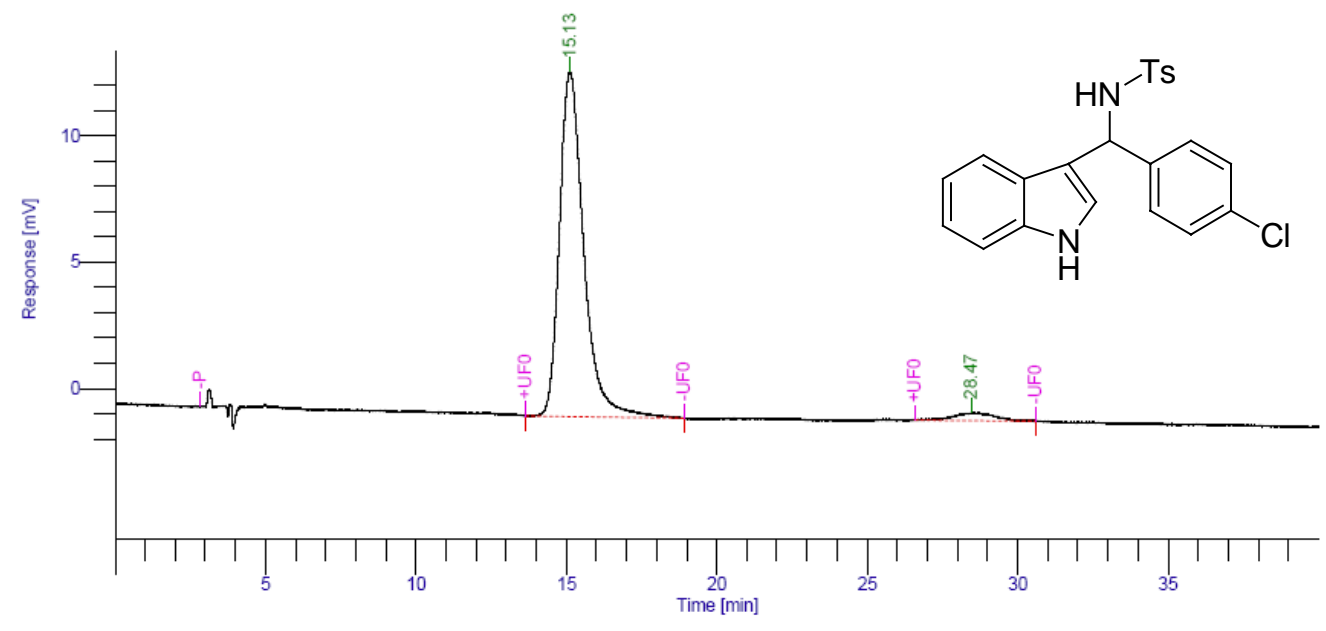

\begin{tabular}{|c|c|c|c|c|c|}
\hline $\begin{array}{c}\text { Peak } \\
\text { \# }\end{array}$ & $\begin{array}{l}\text { Time } \\
\text { [min] }\end{array}$ & $\begin{array}{c}\text { Area } \\
{\left[u V^{*} \text { sec] }\right.}\end{array}$ & $\begin{array}{l}\text { Height } \\
\text { [uV] }\end{array}$ & $\begin{array}{c}\text { Area } \\
{[\%]}\end{array}$ & $\begin{array}{c}\text { Norm. Area } \\
{[\%]}\end{array}$ \\
\hline \multirow{3}{*}{$\frac{1}{2}$} & 15.13 & 765347.7043 & $1.363 e+04$ & 96.32 & 96.32 \\
\hline & 28.4 & 29221.8892 & 321.4445 & 3.68 & 3.68 \\
\hline & & 794569.5934 & $1.395 e+04$ & 100.00 & 100.00 \\
\hline
\end{tabular}

Chiralcel OD-H Column; Hexi-ProH: 8020 ; Flow rate $=1.0 \mathrm{~mL} / \mathrm{min} ; 254 \mathrm{~nm}$; 


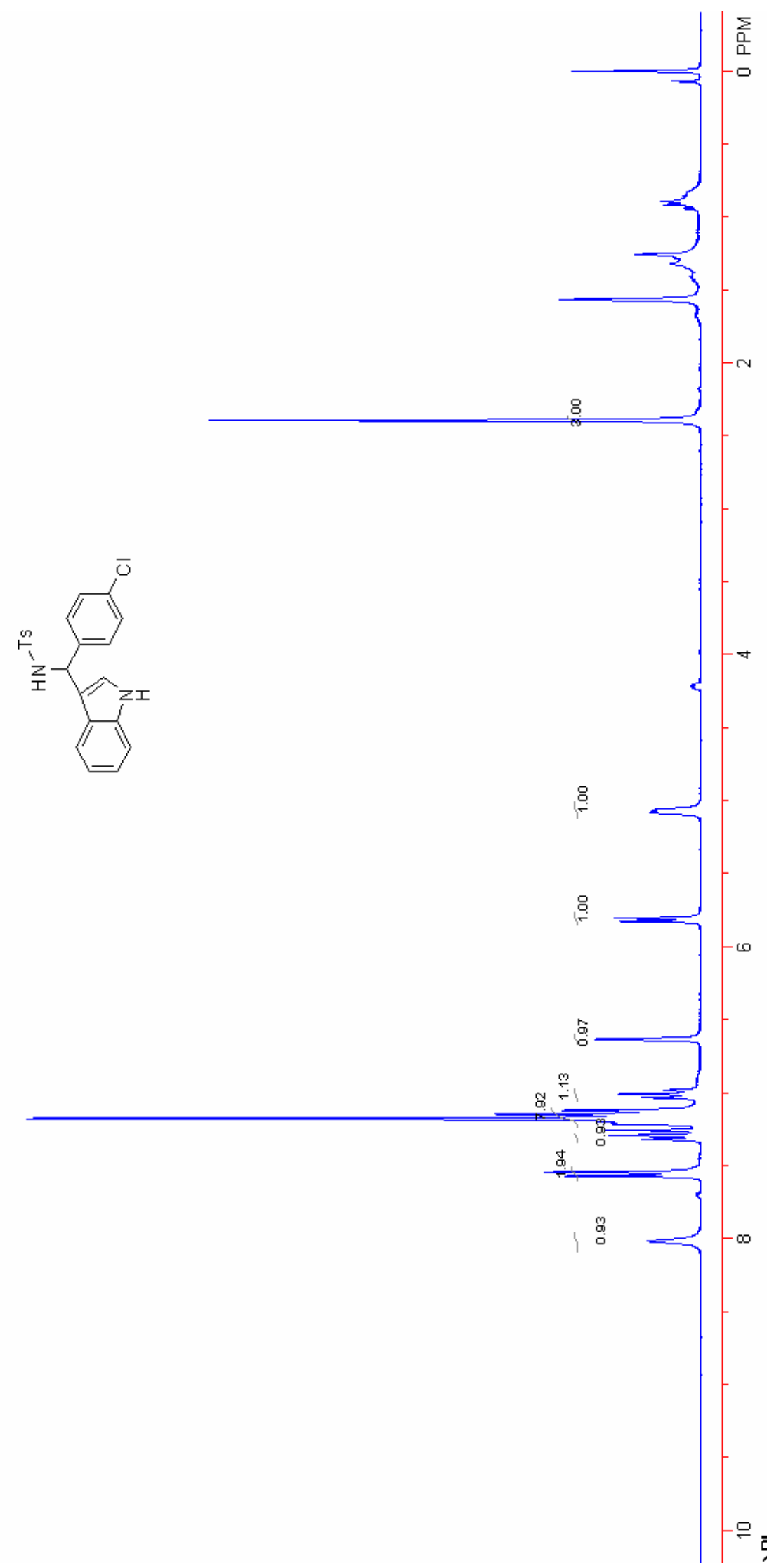




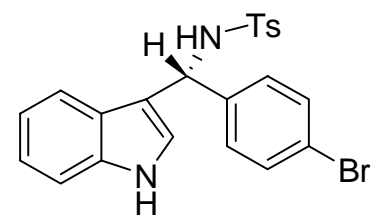

(entry 14, Table 3): $\mathrm{R}_{\mathrm{f}}=0.30$ (ethyl acetate/petroleum ether $=1 / 2, \mathrm{v} / \mathrm{v}$ ); colorless solid, $71 \%$ yield, $82 \%$ ee [Daicel Chiralcel OD-H, Hexanes / IPA $=80 / 20,1.0 \mathrm{ml} \square \mathrm{min}^{-1}, \lambda=254 \mathrm{~nm}, \mathrm{t}$ (major) $=14.59 \mathrm{~min}, \mathrm{t}$ $($ minor $)=28.59 \mathrm{~min}] ;[\mathrm{\alpha}]_{\mathrm{D}}{ }^{20}=+12.4^{\mathrm{o}}(\mathrm{c}=1.35, \mathrm{DCM}) .{ }^{1} \mathrm{H} \mathrm{NMR}\left(300 \mathrm{MHz}, \mathrm{CDCl}_{3}\right) \delta 2.40(\mathrm{~s}, 3 \mathrm{H}), 5.02(\mathrm{~d}$, $J=6.0 \mathrm{~Hz}, 1 \mathrm{H}), 5.80(\mathrm{~d}, J=6.6 \mathrm{~Hz}, 1 \mathrm{H}), 6.64(\mathrm{~s}, 1 \mathrm{H}), 7.01(\mathrm{t}, J=7.2 \mathrm{~Hz}, 1 \mathrm{H}), 7.13-7.21(\mathrm{~m}, 6 \mathrm{H}), 7.13-7.21$ $(\mathrm{m}, 3 \mathrm{H}), 7.56(\mathrm{~d}, J=7.8 \mathrm{~Hz}, 2 \mathrm{H}), 8.01(\mathrm{br}, 1 \mathrm{H})$. 


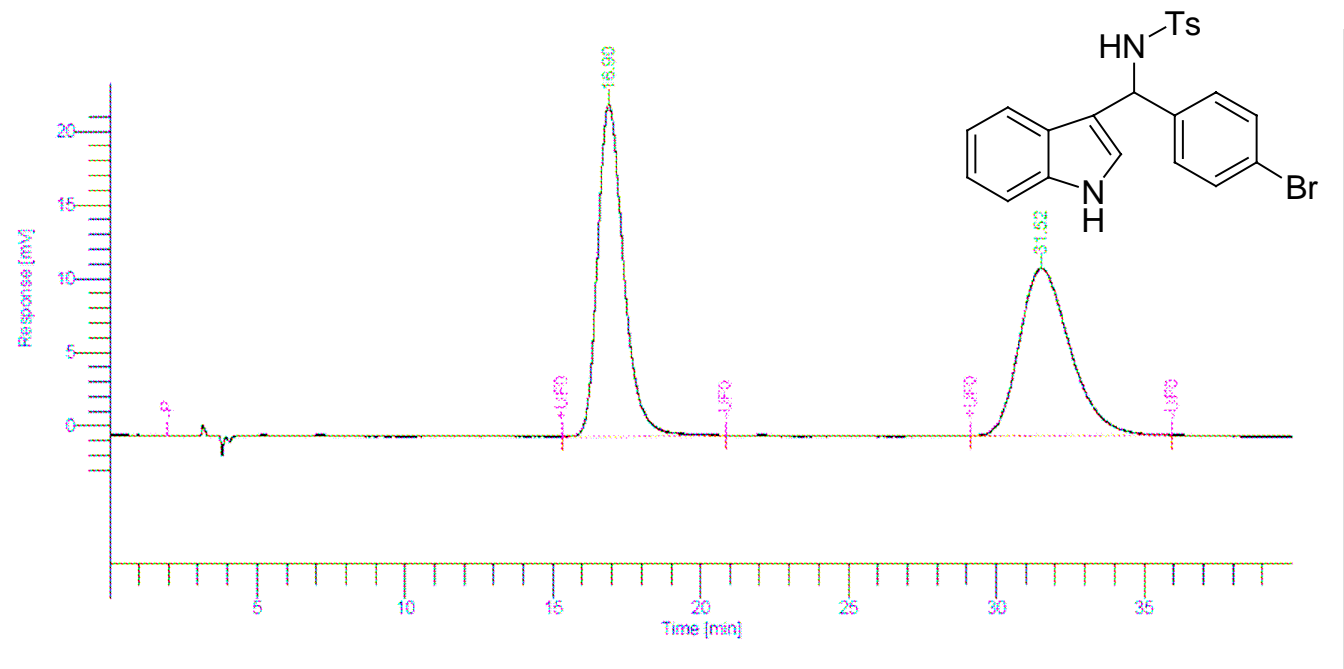

\begin{tabular}{|c|c|c|c|c|c|}
\hline $\begin{array}{c}\text { Peak } \\
\#\end{array}$ & $\begin{array}{l}\text { Time } \\
\text { [min] }\end{array}$ & $\begin{array}{c}\text { Area } \\
{\left[u V^{*} \text { sec }\right]}\end{array}$ & $\begin{array}{l}\text { Height } \\
{[\mathrm{uV}]}\end{array}$ & $\begin{array}{c}\text { Area } \\
{[\%]}\end{array}$ & $\begin{array}{c}\text { Norm. Area } \\
{[\%]}\end{array}$ \\
\hline \multirow{3}{*}{$\begin{array}{l}1 \\
2\end{array}$} & 16.90 & $1.3824 \mathrm{e}+06$ & $2.256 \mathrm{e}+04$ & 50.52 & 50.52 \\
\hline & 31.52 & $1.3541 \mathrm{e}+06$ & $1.138 \mathrm{e}+04$ & 49.48 & 49.48 \\
\hline & & $2.7365 e+06$ & $3.394 e+04$ & 100.00 & 100.00 \\
\hline
\end{tabular}

Chiralcel OD-H Column; Hex/i-PrOH: 80/20; Flow rate $=1.0 \mathrm{~mL} / \mathrm{min} ; 254$ nm;

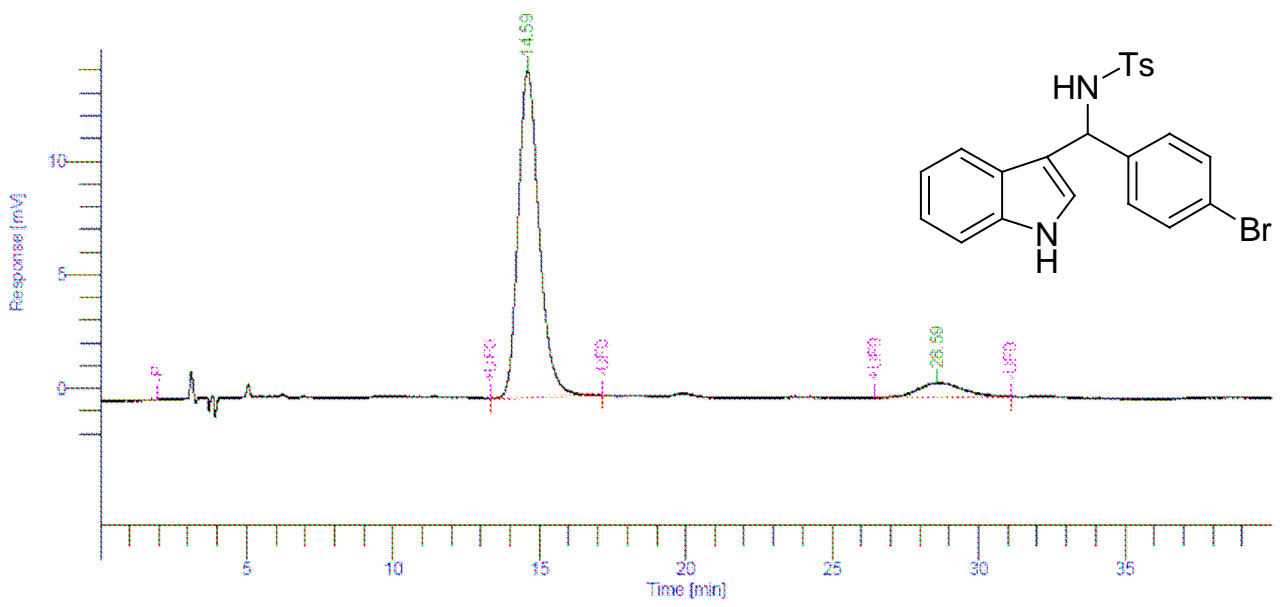

\begin{tabular}{|c|c|c|c|c|c|}
\hline $\begin{array}{c}\text { Peak } \\
\text { 亲 }\end{array}$ & $\begin{array}{l}\text { Time } \\
\text { [min] }\end{array}$ & $\begin{array}{c}\text { Area } \\
{\left[\mathrm{uV}^{*} \mathrm{sec}\right]}\end{array}$ & $\begin{array}{l}\text { Height } \\
{[u v]}\end{array}$ & $\begin{array}{c}\text { Area } \\
{[\%]}\end{array}$ & $\begin{array}{c}\text { Norm. Area } \\
{[\%]}\end{array}$ \\
\hline \multirow{3}{*}{$\begin{array}{l}1 \\
2\end{array}$} & 14.59 & 718120.6813 & $1.441 e^{+04}$ & 91.39 & 91.39 \\
\hline & 28.59 & 67653.1993 & 652.4321 & 8.61 & 8.61 \\
\hline & & 785773.8807 & $1.506 e^{+04}$ & 100.00 & 100.00 \\
\hline
\end{tabular}

Chiralcel OD-H Column; Hex/I-PrOH: $80 / 20$; Flow rate $=1.0 \mathrm{~mL} / \mathrm{min} ; 254 \mathrm{~nm}$; 
$\operatorname{tot} z-$
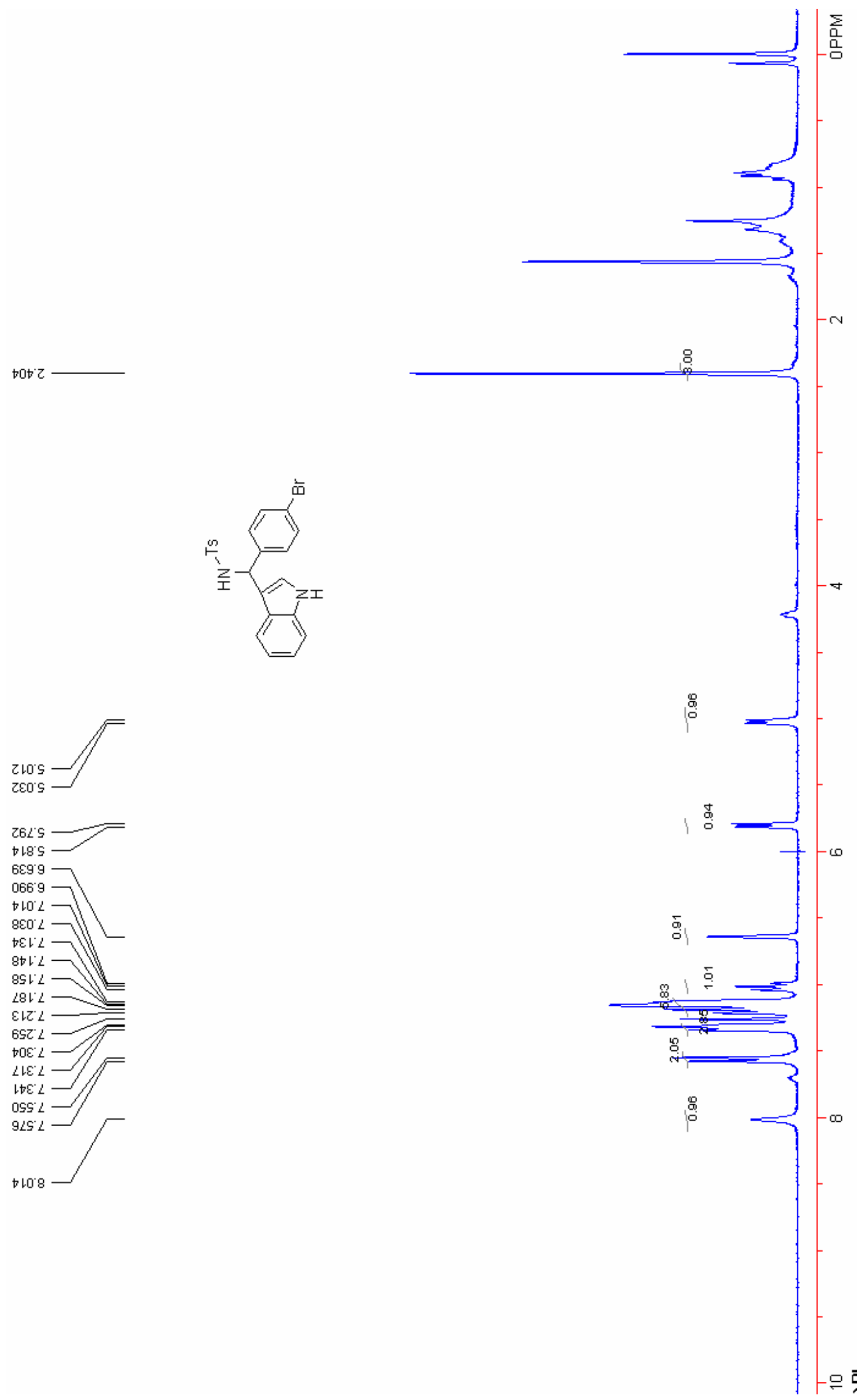


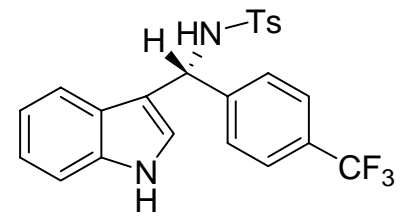

(entry 15, Table 3): $\mathrm{R}_{\mathrm{f}}=0.40$ (ethyl acetate/petroleum ether $=1 / 2, \mathrm{v} / \mathrm{v}$ ); colorless solid, $83 \%$ yield, $85 \%$ ee [Daicel Chiralcel OD-H, Hexanes $/ \mathrm{IPA}=70 / 30,0.8 \mathrm{ml} \square \mathrm{min}^{-1}, \lambda=254 \mathrm{~nm}, \mathrm{t}$ (major) $=11.71 \mathrm{~min}$, $\mathrm{t}($ minor $)=22.04 \mathrm{~min}] ;[\alpha]_{\mathrm{D}}{ }^{20}=+27.5^{\circ}(\mathrm{c}=1.0$, Acetone $) .{ }^{1} \mathrm{H}$ NMR $\left(300 \mathrm{MHz}, \mathrm{DMSO}-\mathrm{d}_{6}\right) \delta 2.26(\mathrm{~s}, 3 \mathrm{H})$, $5.83(\mathrm{~d}, J=9.0 \mathrm{~Hz}, 1 \mathrm{H}), 6.77(\mathrm{~d}, J=2.4 \mathrm{~Hz}, 1 \mathrm{H}), 6.93(\mathrm{t}, J=7.5 \mathrm{~Hz}, 1 \mathrm{H}), 7.04-7.11(\mathrm{~m}, 3 \mathrm{H}), 7.31-7.39(\mathrm{~m}$, $2 \mathrm{H}), 7.45-7.48(\mathrm{~m}, 6 \mathrm{H}), 8.61(\mathrm{~d}, J=9.0 \mathrm{~Hz}, 1 \mathrm{H}), 10.96(\mathrm{br}, 1 \mathrm{H}) ;{ }^{13} \mathrm{C}$ NMR $\left(75 \mathrm{MHz}, \mathrm{DMSO}-\mathrm{d}_{6}\right) \delta 20.8$, $54.2,110.9,115.9,118.8,120.2,124.1,124.9,125.9,126.2,126.7,126.9,127.9,128.8,136.7,138.6,141.3$, 141.8 . 


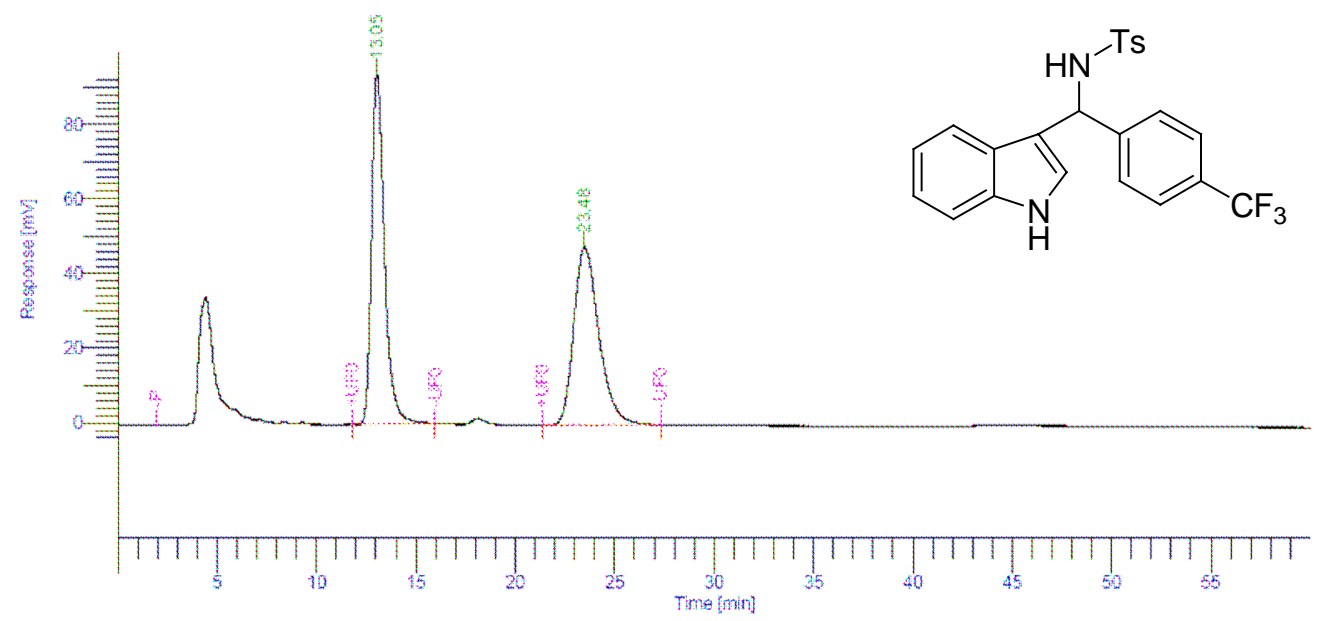

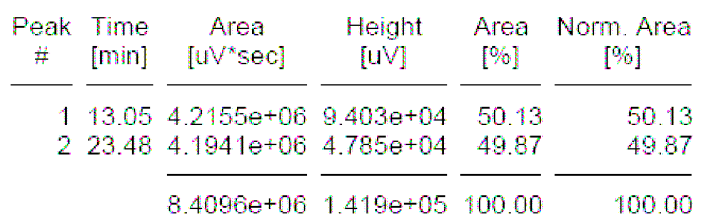

Chiralcel OD-H Column; Hex/-PrOH: $70 / 30$; Flow rate $=0.8 \mathrm{~mL} / \mathrm{min} ; 220 \mathrm{~nm}$;

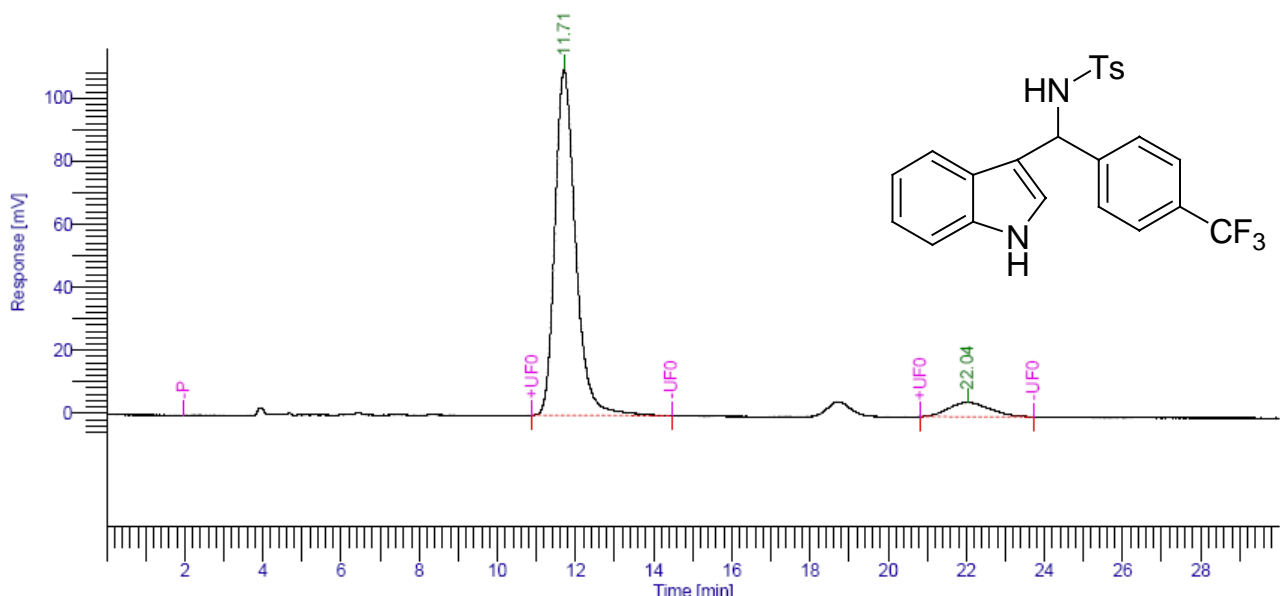

\begin{tabular}{|c|c|c|c|c|c|}
\hline $\begin{array}{c}\text { Peak } \\
\#\end{array}$ & $\begin{array}{l}\text { Time } \\
\text { [min] }\end{array}$ & $\begin{array}{c}\text { Area } \\
{\left[\mathrm{u}^{*} \mathrm{sec}\right]}\end{array}$ & $\begin{array}{l}\text { Height } \\
{[\mathrm{uV}]}\end{array}$ & $\begin{array}{c}\text { Area } \\
{[\%]}\end{array}$ & $\begin{array}{c}\text { Norm. Area } \\
{[\%]}\end{array}$ \\
\hline 1 & 11.71 & $4.0479 e+06$ & $1.099 e^{+05}$ & 92.43 & 92.43 \\
\hline 2 & 22.04 & 331558.9606 & 4525.9120 & 7.57 & 7.57 \\
\hline
\end{tabular}

Chiralcel OD-H Column; Hexi-FrOH: 70/30; Flow rate $=0.8 \mathrm{~mL} / \mathrm{min} ; 220 \mathrm{~nm}$; 


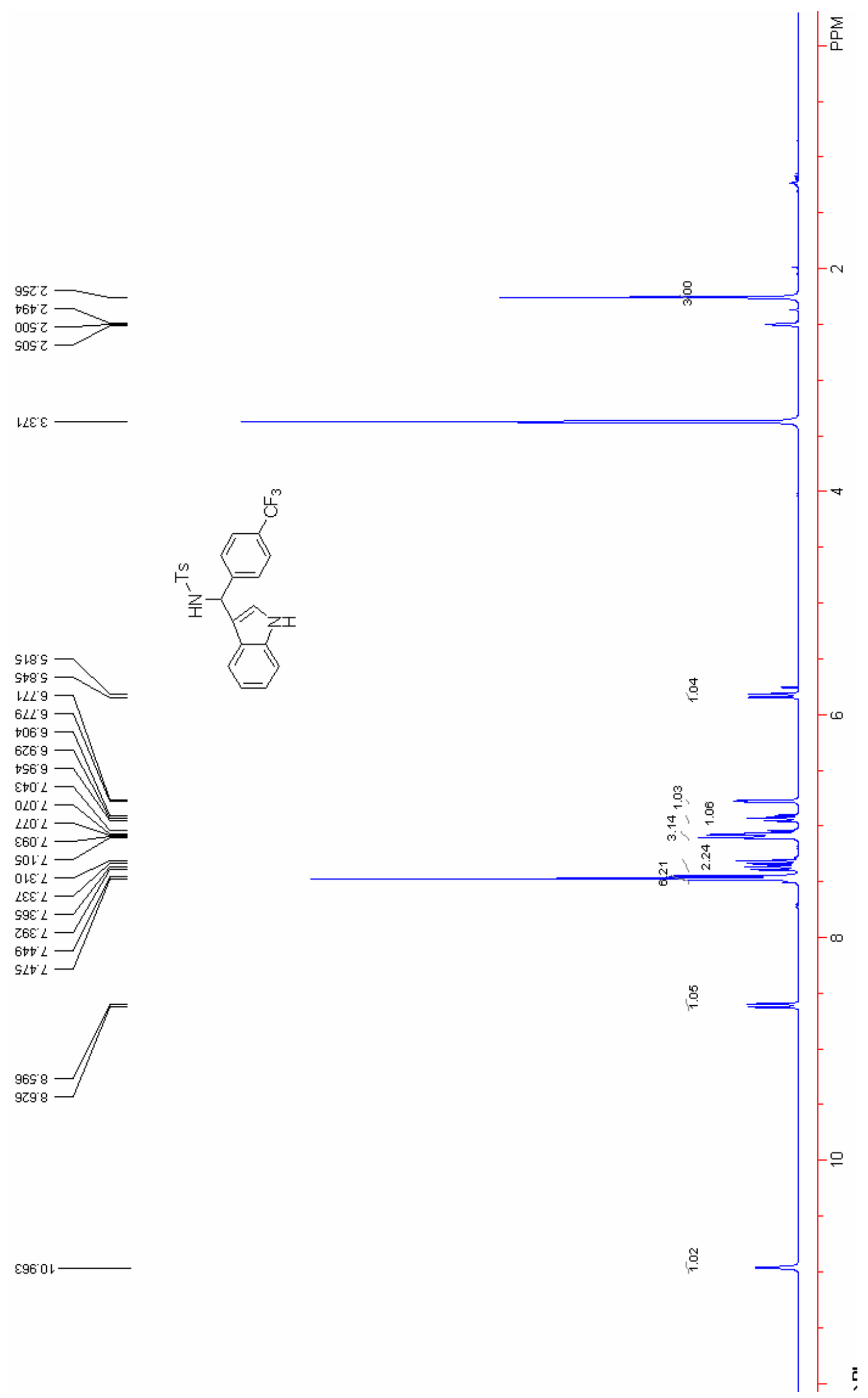



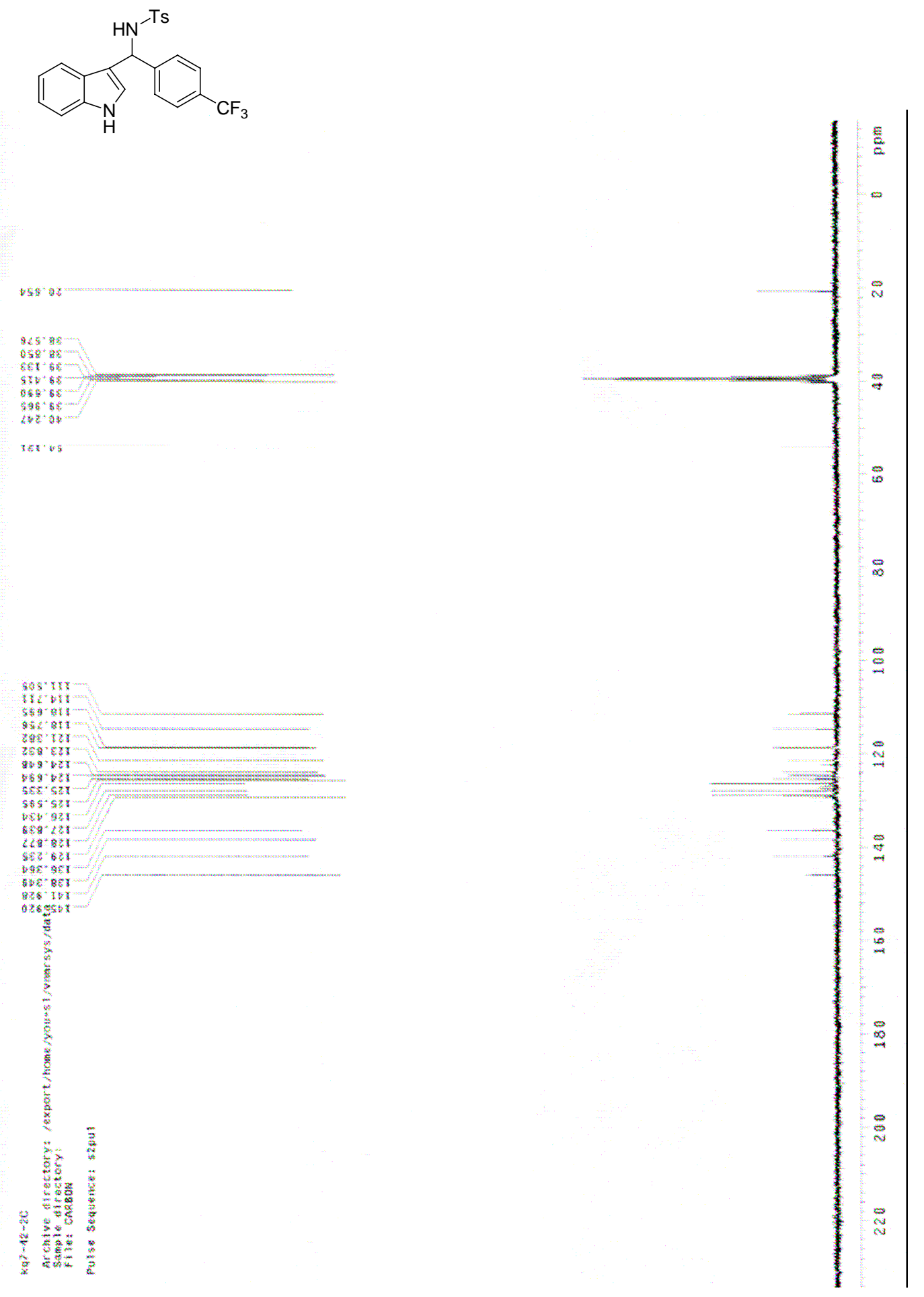


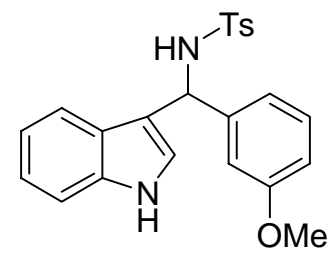

(entry 16, Table 3): $R_{\mathrm{f}}=0.30$ (ethyl acetate/petroleum ether $=1 / 2$, v/v); colorless solid, $90 \%$ yield, $96 \%$ ee [Daicel Chiralcel OD-H, Hexanes / IPA $=80 / 20,0.7 \mathrm{ml} \square \mathrm{min}^{-1}, \lambda=230 \mathrm{~nm}, \mathrm{t}$ (major) $=12.31 \mathrm{~min}, \mathrm{t}$ $($ minor $)=21.63 \mathrm{~min}] ;[\alpha]_{\mathrm{D}}{ }^{20}=+12.3^{\circ}(\mathrm{c}=1.03$, Acetone $) .{ }^{1} \mathrm{H}$ NMR $\left(300 \mathrm{MHz}, \mathrm{CDCl}_{3}\right) \delta 2.31(\mathrm{~s}, 3 \mathrm{H}), 3.62$ $(\mathrm{s}, 3 \mathrm{H}), 5.44(\mathrm{~d}, J=6.9 \mathrm{~Hz}, 1 \mathrm{H}), 5.78(\mathrm{~d}, J=7.2 \mathrm{~Hz}, 1 \mathrm{H}), 6.58(\mathrm{~s}, 1 \mathrm{H}), 6.67-6.70(\mathrm{~m}, 2 \mathrm{H}), 6.76(\mathrm{~d}, J=7.5$ $\mathrm{Hz}, 1 \mathrm{H}), 6.93-7.13(\mathrm{~m}, 5 \mathrm{H}), 7.20-7.28(\mathrm{~m}, 2 \mathrm{H}), 7.48$ (d, J=8.1 Hz, 2H), 8.14 (br, 1H); ${ }^{13} \mathrm{C} \mathrm{NMR}(75 \mathrm{MHz}$, $\left.\mathrm{CDCl}_{3}\right) \delta 21.3,54.9,55.0,111.3,112.5,112.9,115.9,119.1,119.6,119.7,122.2,123.8,125.3,127.0,129.1$, $129.2,136.4,137.3,141.8,142.9,159.4$. 

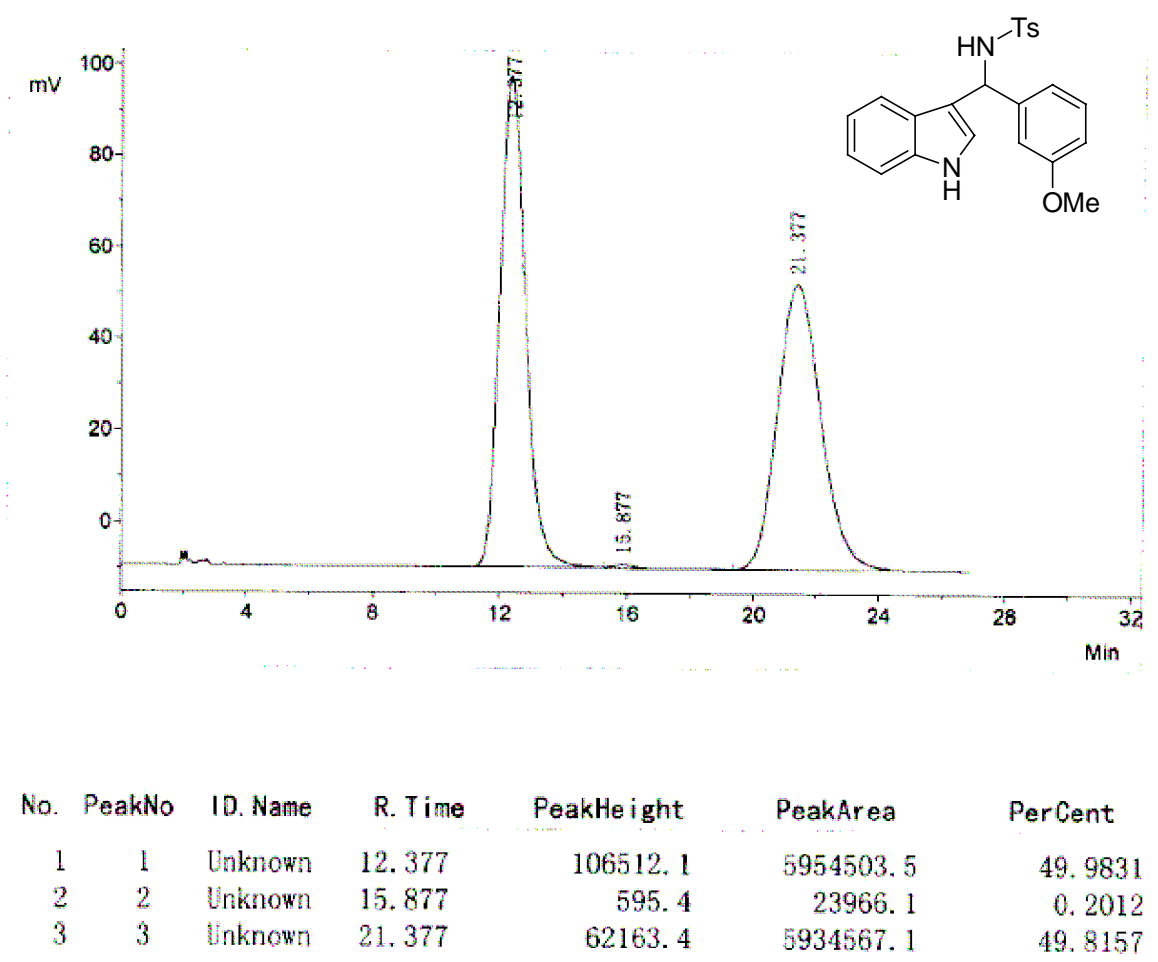

Percent

Total

$\begin{array}{lll}169270.9 & 11913036.7 & 100.0000\end{array}$

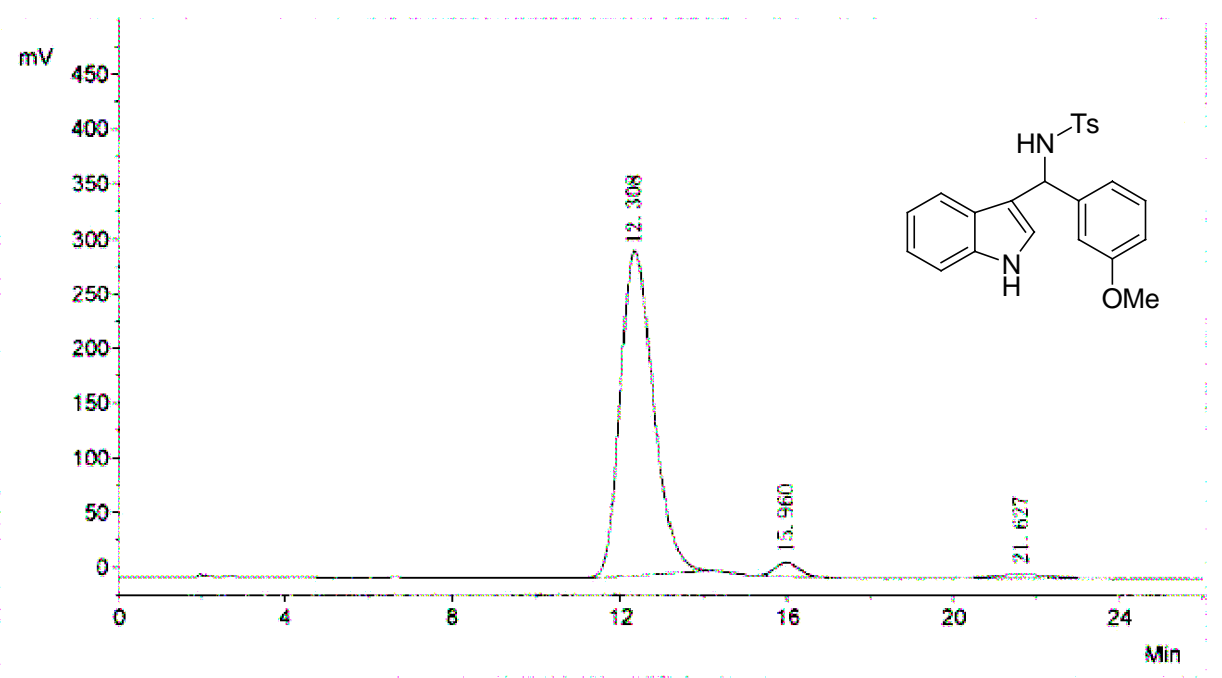

\begin{tabular}{cccrrrr} 
No. PeakNo & 10. Name & R. Time & PeakHeight & \multicolumn{1}{c}{ PeakArea } & \multicolumn{1}{c}{ PerCent } \\
1 & 1 & Unknown & 12.308 & 292746.6 & 16174793.4 & 94.9319 \\
2 & 2 & Unknown & 15.960 & 12379.3 & 494786.4 & 2.9040 \\
3 & 3 & Unknown & 21.627 & 3892.7 & 368737.6 & 2.1642 \\
& & & & 309018.6 & 17038317.4 & 100.0000
\end{tabular}


$60 \varepsilon z-$

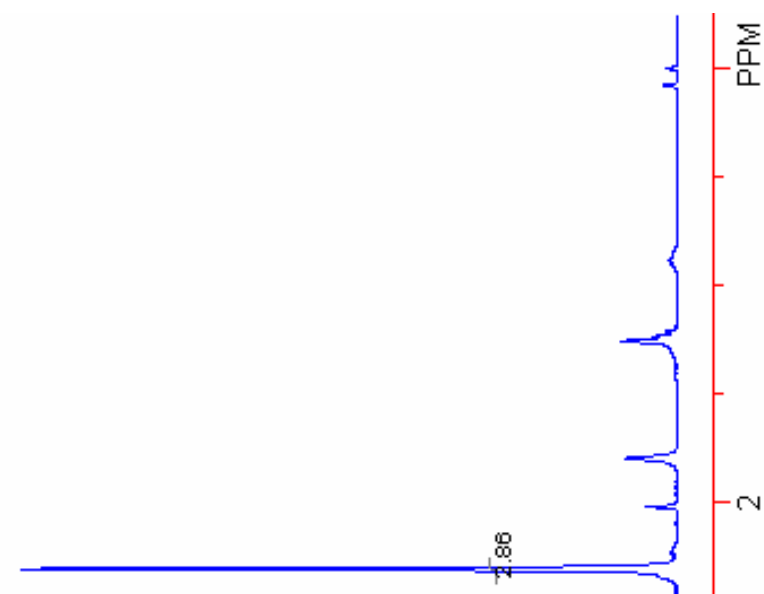

$\operatorname{sig} \varepsilon$

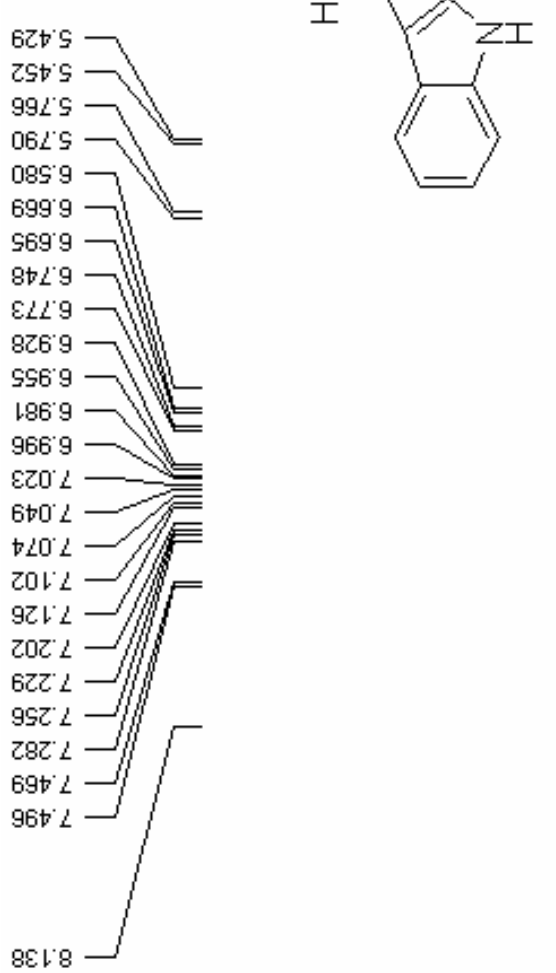

8

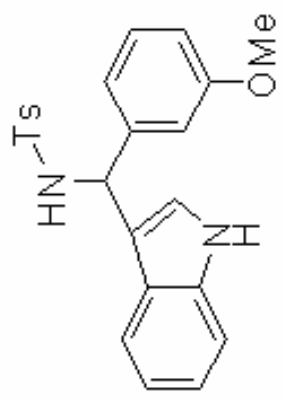

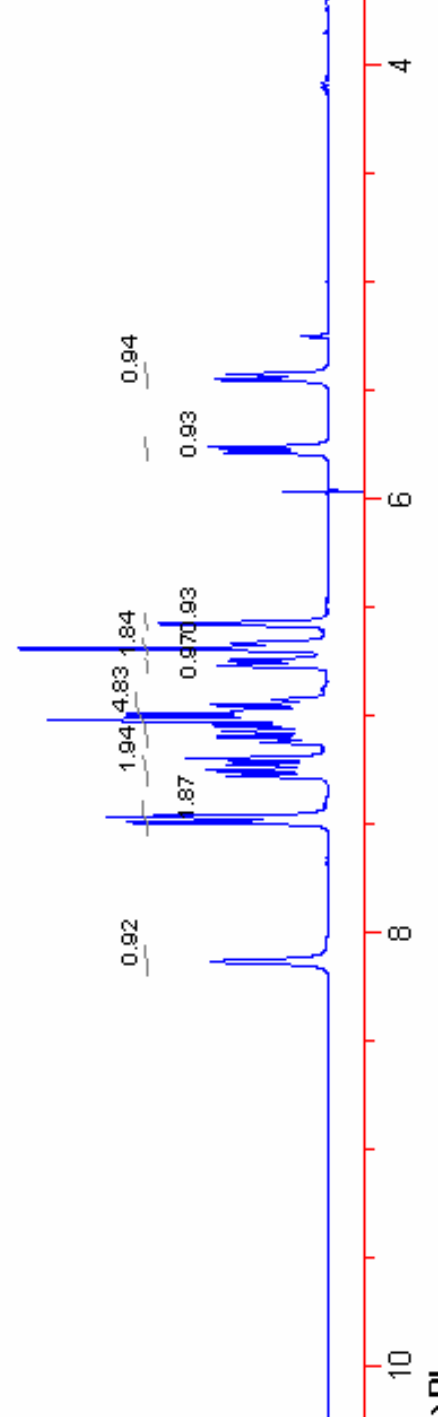




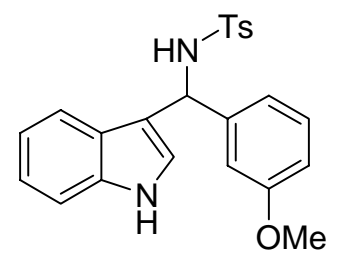

SEะ " 12

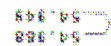
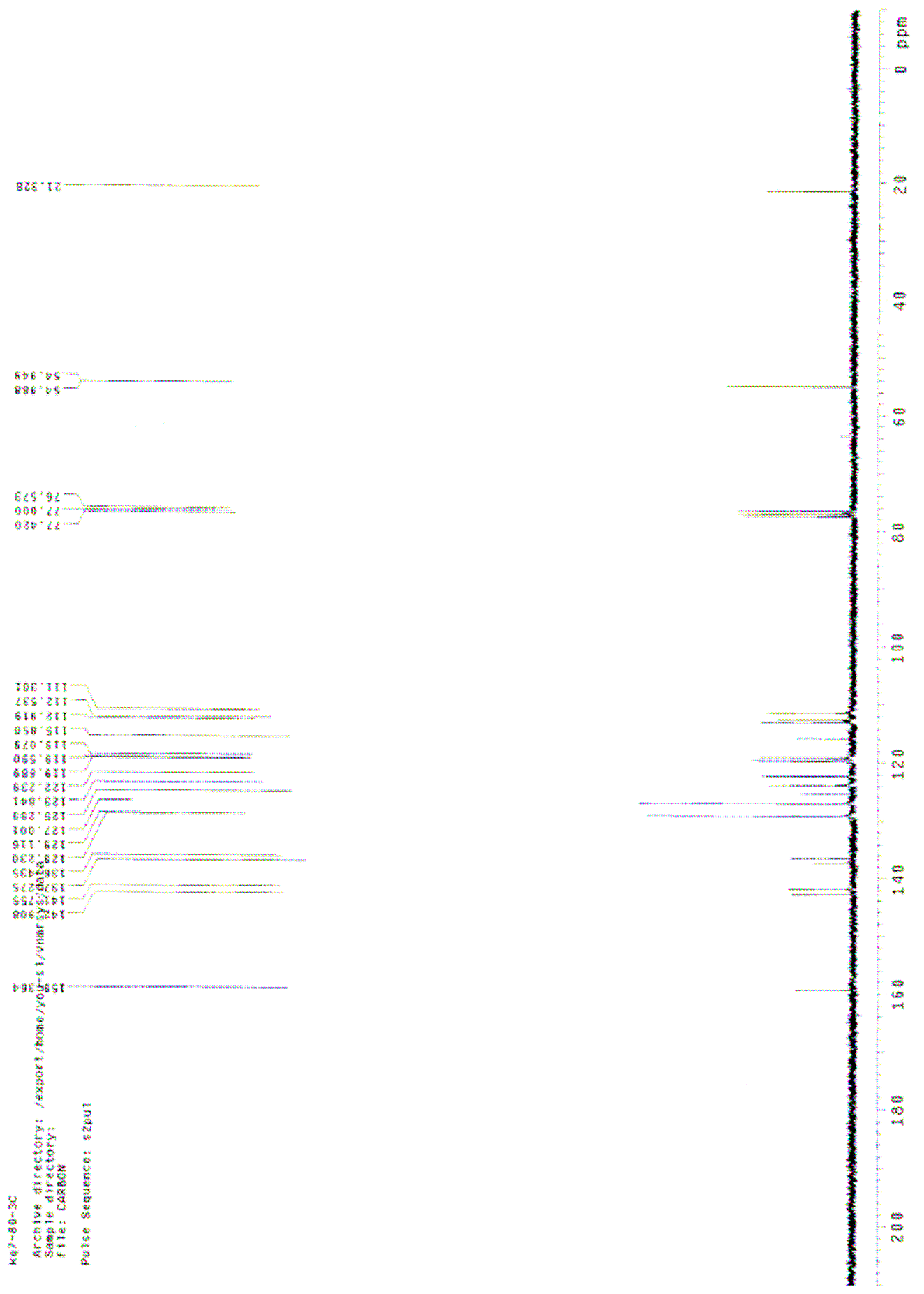


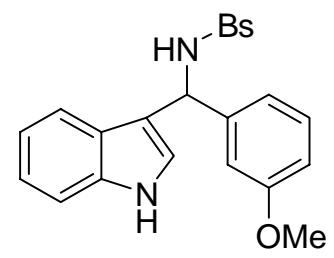

(entry 17 , Table 3 ): $R_{\mathrm{f}}=0.30$ (ethyl acetate/petroleum ether $=1 / 2$, v/v); colorless solid, $90 \%$ yield, 97\% ee [Daicel Chiralcel OD-H, Hexanes / IPA $=70 / 30,0.7 \mathrm{ml} \square \mathrm{min}^{-1}, \lambda=230 \mathrm{~nm}, \mathrm{t}$ (major) $=13.21$ $\min , \mathrm{t}($ minor $)=21.21 \mathrm{~min}] ;[\alpha]_{\mathrm{D}}{ }^{20}=+12.7^{\circ}(\mathrm{c}=0.84$, Acetone $) .{ }^{1} \mathrm{H} \mathrm{NMR}\left(300 \mathrm{MHz}, \mathrm{CDCl}_{3}\right) \delta 3.66(\mathrm{~s}$, $3 \mathrm{H}), 5.27(\mathrm{~d}, J=7.2 \mathrm{~Hz}, 1 \mathrm{H}), 5.55(\mathrm{~d}, J=6.9 \mathrm{~Hz}, 1 \mathrm{H}), 6.63(\mathrm{~s}, 1 \mathrm{H}), 6.70-6.74(\mathrm{~m}, 2 \mathrm{H}), 6.80(\mathrm{~d}, J=7.8 \mathrm{~Hz}$, 1H), 6.98-7.18 (m, 3H), 7.26-7.32 (m, 4H), 7.43 (t, $J=7.2 \mathrm{~Hz}, 1 \mathrm{H}), 7.75$ (d, J=7.8 Hz, 2H), 8.02 (br, 1H); ${ }^{13} \mathrm{C}$ NMR $\left(75 \mathrm{MHz}, \mathrm{CDCl}_{3}\right) \delta 55.0,55.1,111.3,112.6,113.1,116.1,119.2,119.6,119.9,122.5,123.8$, 125.3, 127.0, 128.6, 129.3, 132.2, 136.5, 140.4, 141.6, 159.5; MS (EI): m/z (\%relative intensity) $392\left(\mathrm{M}^{+}\right.$, 15), 235 (100), 77 (97), 157 (50), 93 (37). HRMS Calcd for $\left(\mathrm{M}^{+}\right) \mathrm{C}_{22} \mathrm{H}_{20} \mathrm{~N}_{2} \mathrm{O}_{3} \mathrm{~S}$ : 392.1161. Found: 392.1176. 


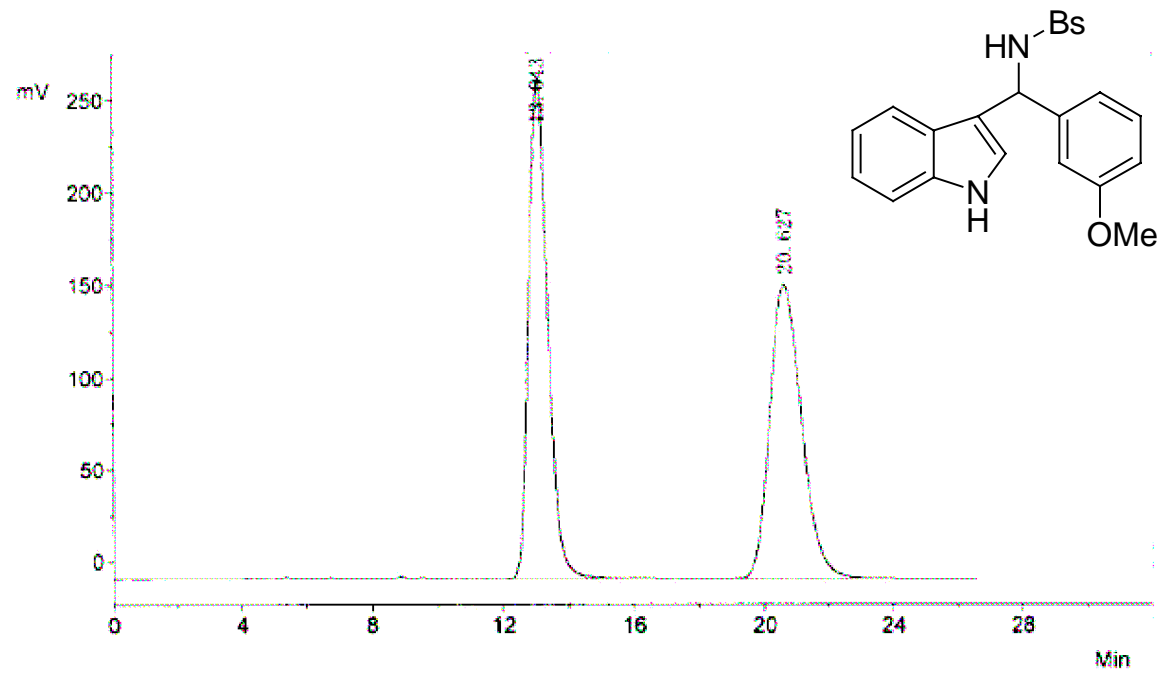

$\begin{array}{cccrrrr}\text { No. PeakNo } & \text { ID. Name } & \text { R. Time } & \text { PeakHeight } & \text { PeakArea } & \text { PerCent } \\ 1 & 1 & \text { Unknown } & 13.043 & 270786.3 & 10821851.2 & 49.8225 \\ 2 & 2 & \text { Unknown } & 20.627 & 159319.2 & 10898938.9 & 50.1775 \\ \text { Total } & & & & 430105.4 & 21720790.1 & 100.0000\end{array}$

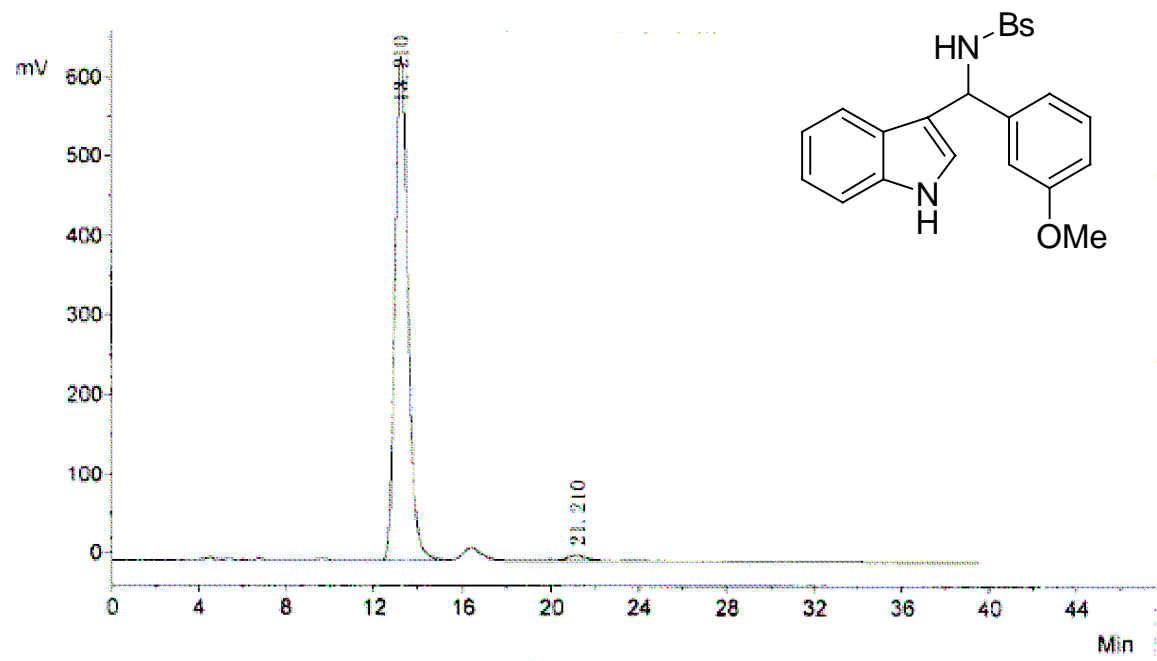

\begin{tabular}{rrrrrrr} 
No PeakNo & ID. Name & R. Time & PeakHeight & PeakArea & \multicolumn{1}{c}{ PerCent } \\
1 & 1 & Unknown & 13.210 & 634274.9 & 26158828.7 & 98.4192 \\
2 & 2 & Unknown & 21.210 & 6127.4 & 420165.0 & 1.5808 \\
Total & & & 640402.4 & 26578993.7 & 100.0000
\end{tabular}




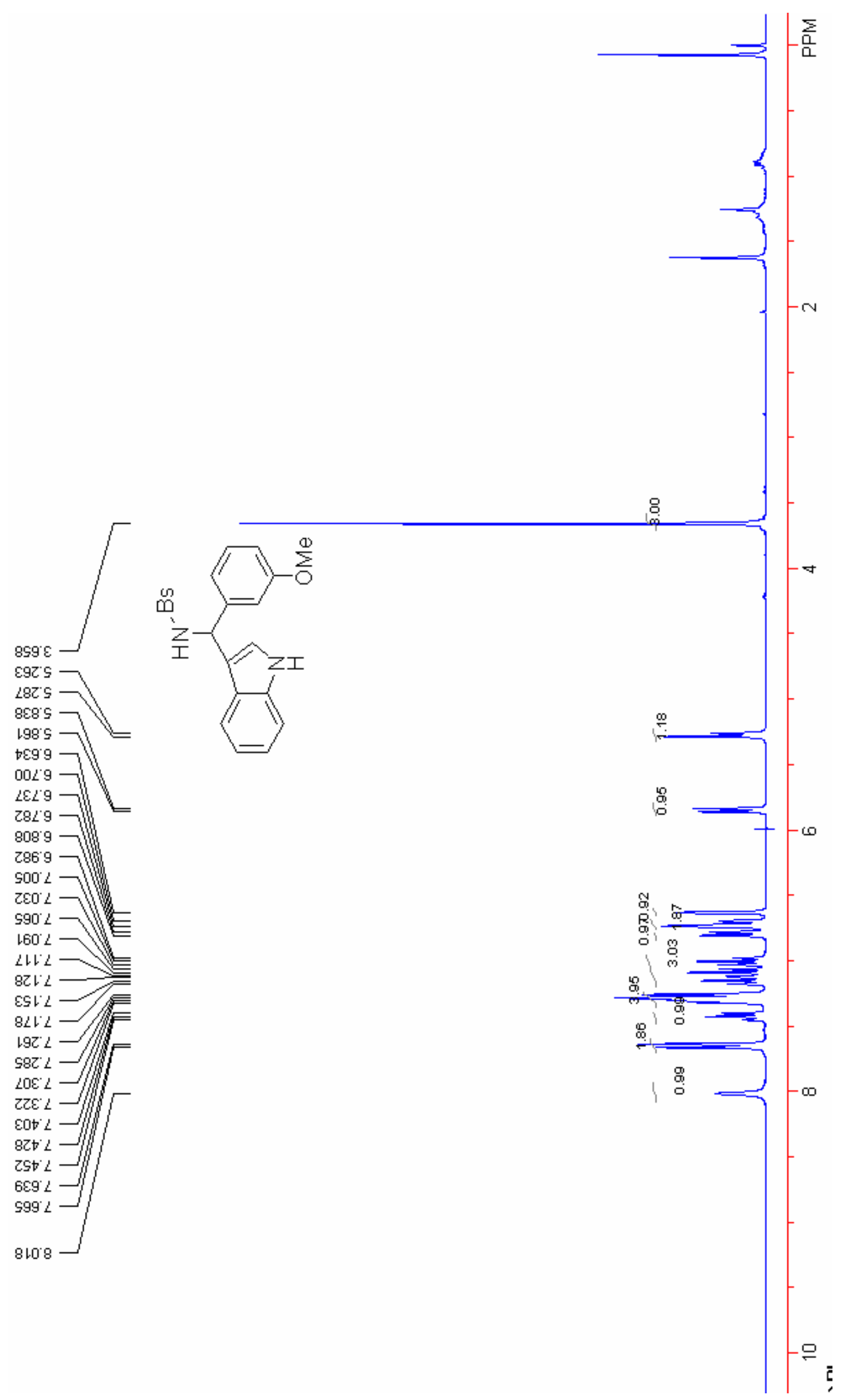


<smiles>COc1cccc(C(NC(=O)c2ccccc2)c2c[nH]c3ccccc23)c1</smiles>

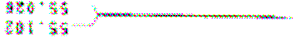

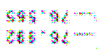

$250.28=$

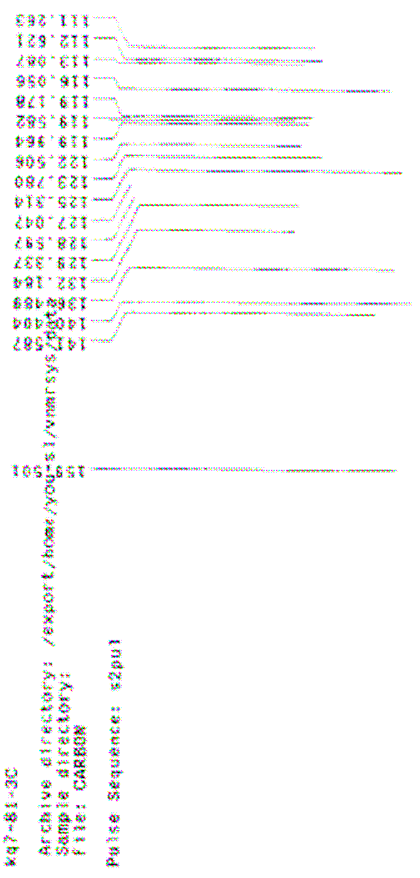

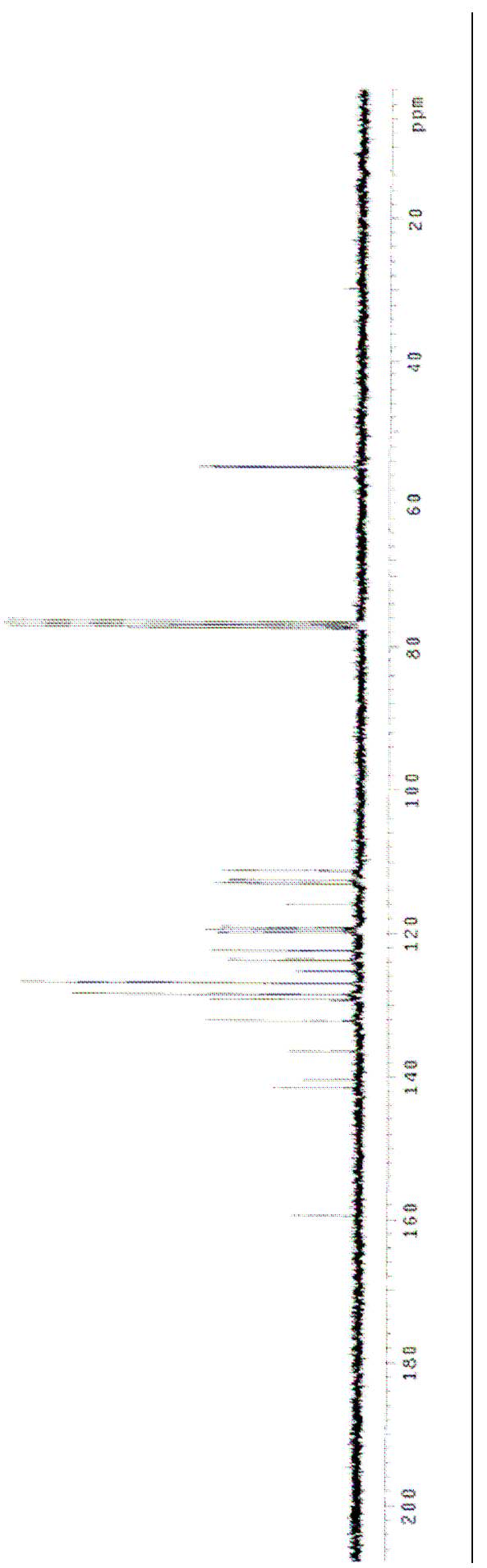




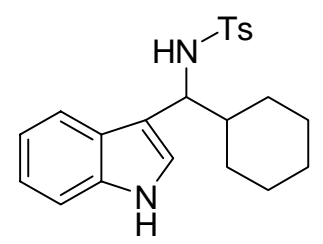

(entry 18, Table 3): $\mathrm{R}_{\mathrm{f}}=0.60$ (ethyl acetate/petroleum ether $=1 / 2, \mathrm{v} / \mathrm{v}$ ); colorless solid, $56 \%$ yield, $58 \%$ ee [Daicel Chiralcel OD-H, Hexanes $/ \mathrm{IPA}=70 / 30,0.7 \mathrm{ml} \square \mathrm{min}^{-1}, \lambda=230 \mathrm{~nm}, \mathrm{t}$ (major) $=10.13 \mathrm{~min}$, $\mathrm{t}($ minor $)=6.96 \mathrm{~min}] ;[\alpha]_{\mathrm{D}}{ }^{20}=-4.2^{\circ}(\mathrm{c}=0.90$, Acetone $) .{ }^{1} \mathrm{H}$ NMR $\left(300 \mathrm{MHz}, \mathrm{CDCl}_{3}\right) \delta 0.81-1.16(\mathrm{~m}, 5 \mathrm{H})$, 1.42-1.46 (m, 1H), 1.57-1.59 (m, 2H), 1.66-1.74 (m, 1H), 1.82-1.85 (m, 1H), 2.00-2.05 (m, 1H), $2.21(\mathrm{~s}$, $3 \mathrm{H}), 4.34(\mathrm{t}, J=8.0 \mathrm{~Hz}, 1 \mathrm{H}), 5.24(\mathrm{~d}, J=7.8 \mathrm{~Hz}, 1 \mathrm{H}), 6.73(\mathrm{~d}, J=2.1 \mathrm{~Hz}, 1 \mathrm{H}), 6.87(\mathrm{~d}, J=7.8 \mathrm{~Hz}, 2 \mathrm{H}), 6.99$ $(\mathrm{t}, J=7.4 \mathrm{~Hz}, 1 \mathrm{H}), 7.10(\mathrm{t}, J=7.6 \mathrm{~Hz}, 1 \mathrm{H}), 7.20(\mathrm{~d}, J=8.4 \mathrm{~Hz}, 1 \mathrm{H}), 7.38-7.42(\mathrm{~m}, 3 \mathrm{H}), 7.95(\mathrm{br}, 1 \mathrm{H}) ;{ }^{13} \mathrm{C}$ NMR $\left(75 \mathrm{MHz}, \mathrm{CDCl}_{3}\right) \delta 21.3,25.9,25.9,26.2,29.8,29.9,42.9,57.1,111.1,114.9,119.1,119.4,121.9$, $122.5,125.4,126.8,128.6,136.1,137.4,142.3$; 


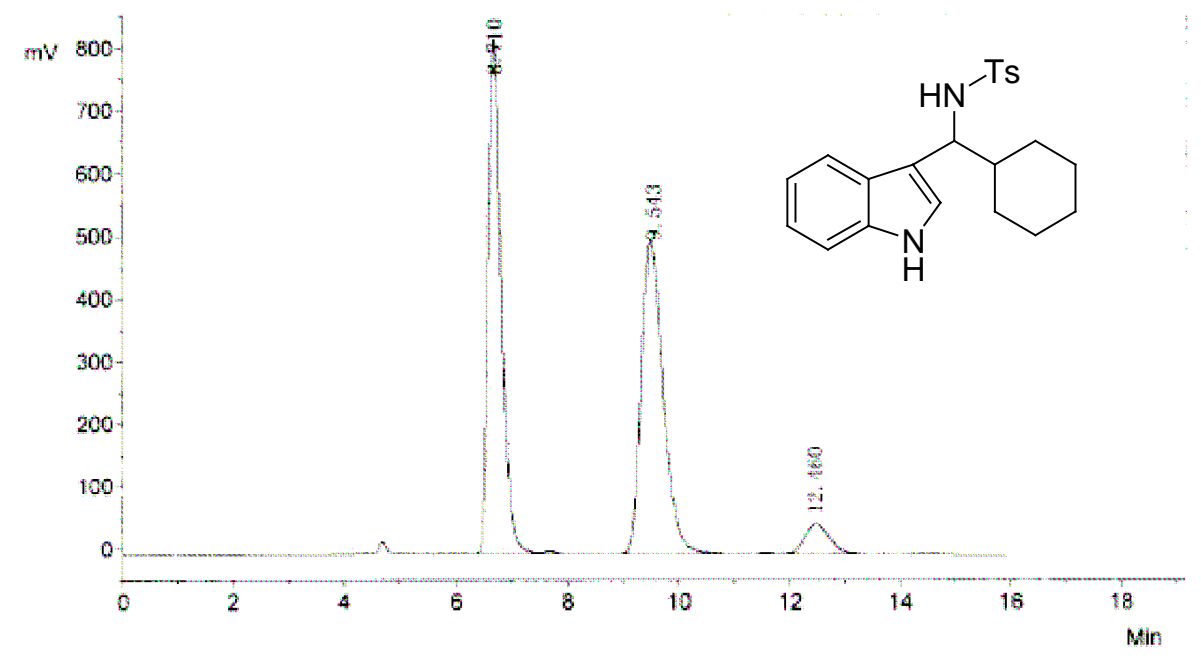

$\begin{array}{rrrrrrr}\text { No PeakNo } & \text { ID Name } & \text { R. Time } & \text { PeakHeight } & \text { PeakArea } & \text { PerGent } \\ 1 & 1 & \text { Lnknown } & 6.710 & 815206.2 & 13738741.5 & 47.4541 \\ 2 & 2 & \text { Lnknown } & 9.543 & 485582.6 & 13759132.7 & 47.5246 \\ 3 & 3 & \text { Lnknown } & 12.460 & 46950.9 & 1453757.4 & 5.0213\end{array}$

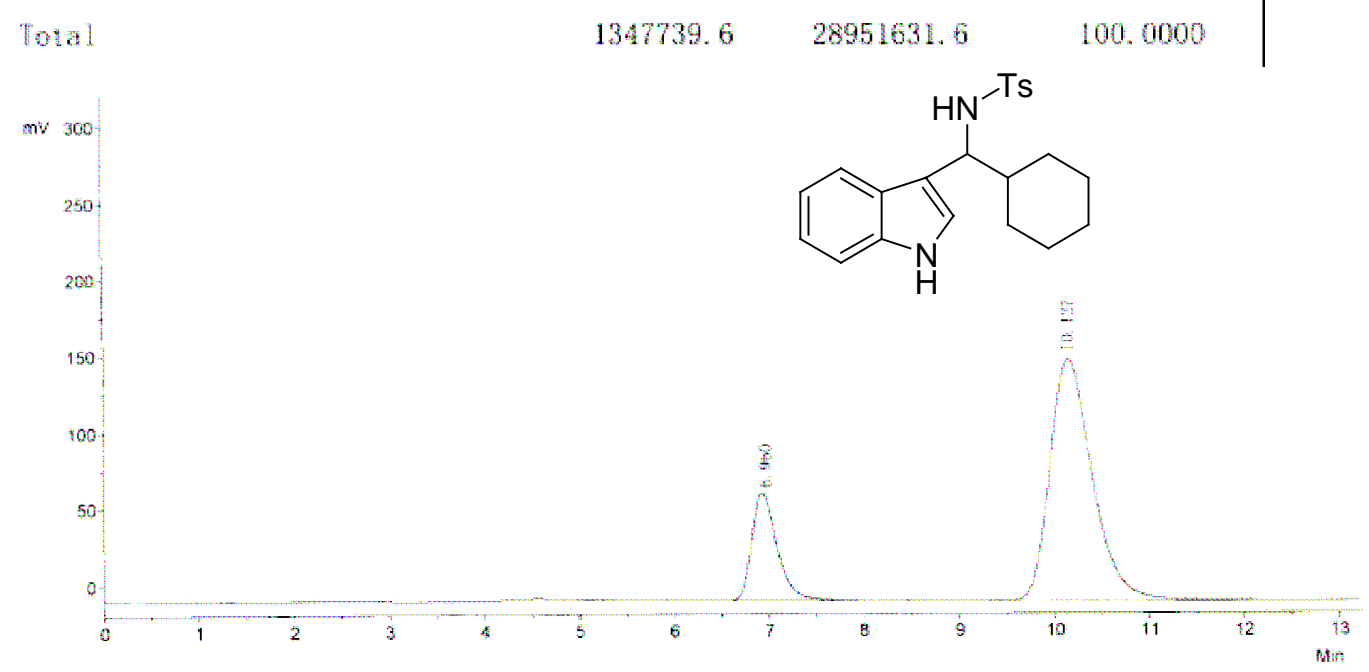

$\begin{array}{rrrr}\text { No. R. Time } & \text { PeakHeight } & \text { PeakArea } & \text { PerCent } \\ 16.960 & 62955.1 & 1300951.5 & 21.1658 \\ 210.127 & 156225.9 & 4845514.1 & 78.8342 \\ \text { Total } & 224191.0 & 6146465.6 & 100.0000\end{array}$




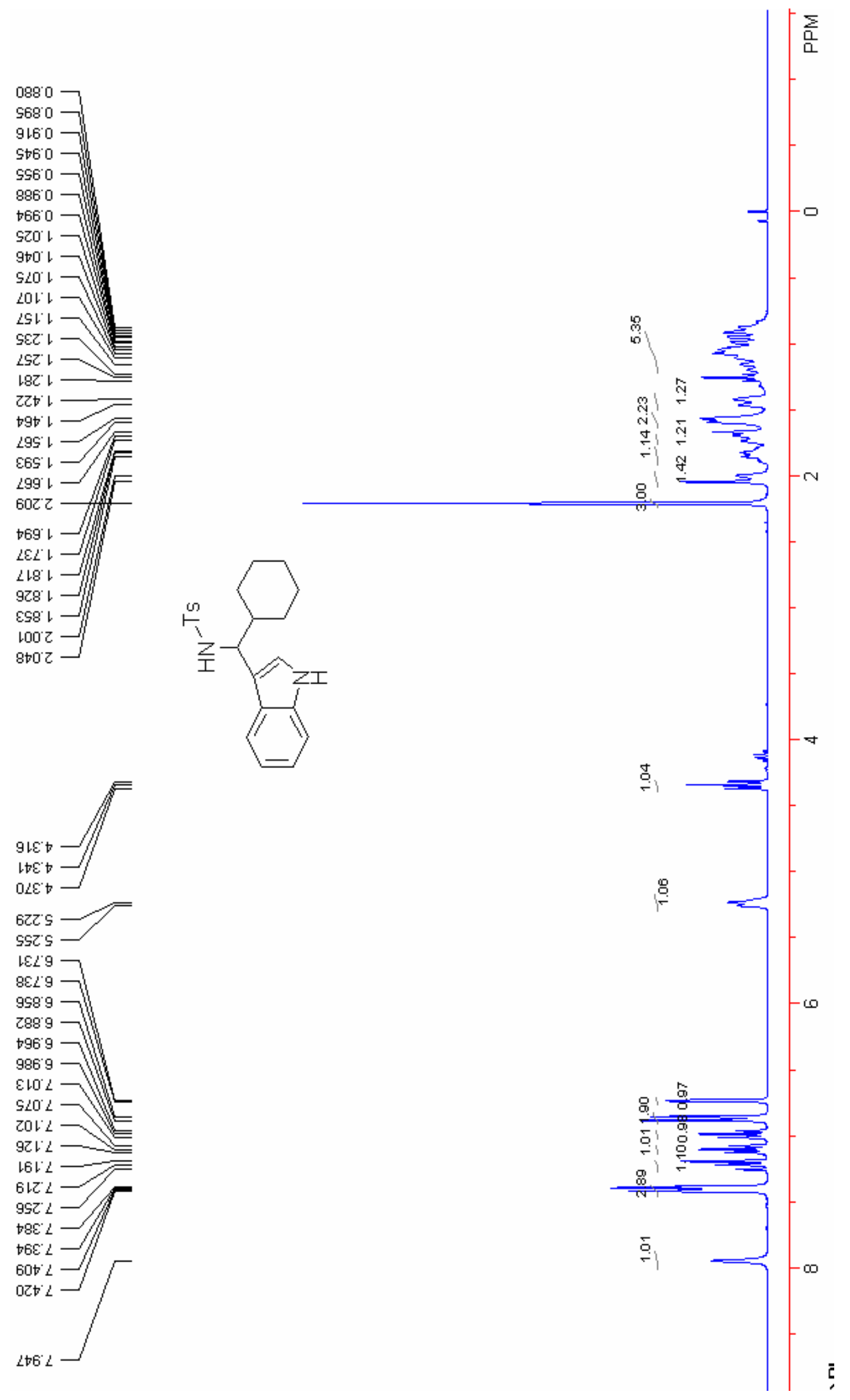



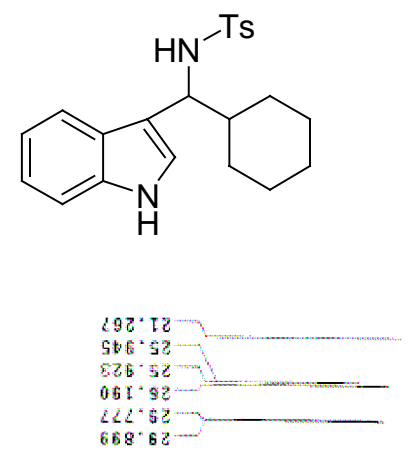

$288^{\circ} 2 t+-$
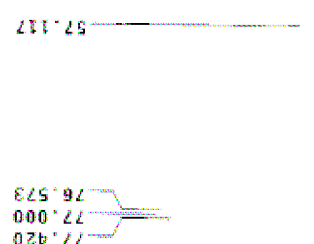

$026: \angle L=$

000.5

-

968.011

$268.61 \mathrm{t}$

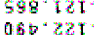

$120 \cdot 521$

दEg. 92 r

$08 \mathrm{~L} \cdot \mathrm{g}$

$98 \varepsilon^{\circ} \angle \varepsilon$

$218 \cdot 2 \mathrm{tat}+\ldots$

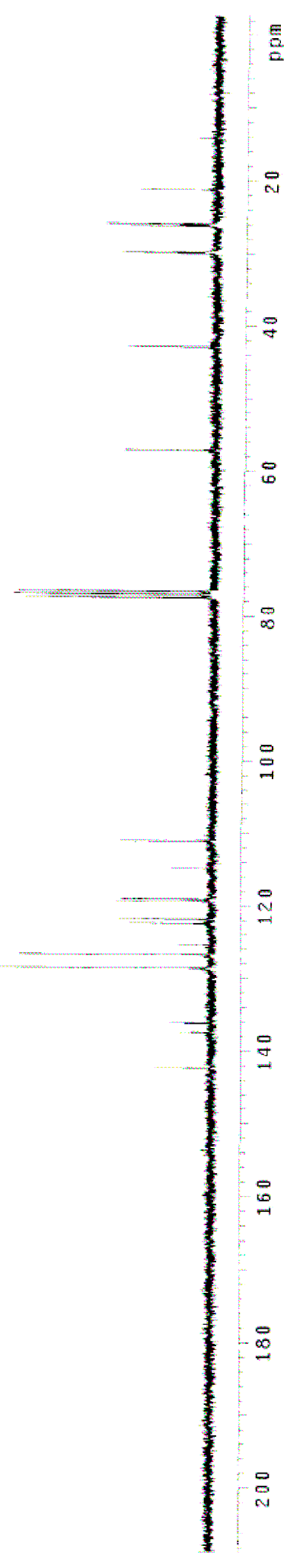

